\title{
DETERMINATION OF THE FERMI SURFACE OF MOLYBDENUM USING THE de HAAS - van ALPHEN EFFECT
}

Ph.D. Thesis Submitted to Iowa State University, May 1972

James Alvin Hoekstra

Ames Laboratory, USAEC

Iowa State University

Ames, Iowa 50010

\section{NOTICE}

This report was prepared as an account of work sponsored by the United States Government. Neither the United States nor the United States Atomic Energy Commission, nor any of their employees, nor any of their contractors, subcontractors, or their employees, makes any warranty, express or implied, or assumes any legal liability or responsibility for the accuracy, completeness or usefulness of any information, apparatus, product or process disclosed, or represents that its use would not infringe privately owned rights.

Date of Manuscript: May 1972 


\section{DISCLAIMER}

This report was prepared as an account of work sponsored by an agency of the United States Government. Neither the United States Government nor any agency Thereof, nor any of their employees, makes any warranty, express or implied, or assumes any legal liability or responsibility for the accuracy, completeness, or usefulness of any information, apparatus, product, or process disclosed, or represents that its use would not infringe privately owned rights. Reference herein to any specific commercial product, process, or service by trade name, trademark, manufacturer, or otherwise does not necessarily constitute or imply its endorsement, recommendation, or favoring by the United States Government or any agency thereof. The views and opinions of authors expressed herein do not necessarily state or reflect those of the United States Government or any agency thereof. 


\section{DISCLAIMER}

Portions of this document may be illegible in electronic image products. Images are produced from the best available original document. 


This report was prepared as an account of work
sponsored by the United States Government. Neither
the United States nor the United States Atomic
Energy Commission, nor any of their employees, nor
any of their contractors, subcontractors, or their
employees, makes any warranty, express or implied,
or assumes any legal liability or responsibility for the
accuracy, completeness or usefulness of any
information, apparatus, product or process disclosed,
or represents that its use would not infringe privately
owned rights.

Available from: National Technical Information Service Department A

Springfield, VA 22151

Price: Microfiche $\$ 0.95$ 


\title{
Determination of the Fermi surface of molybdenum using the de Haas-van Alphen effect
}

by

\author{
James Alvin Hoekstra \\ A Dissertation Submitted to the \\ Graduate Faculty in Partial Fulfillment of \\ The Requirements for the Degree of \\ DOCTOR OF PHILOSOPHY \\ Department: Physics \\ Major: Solid State Physics
}

Approved:

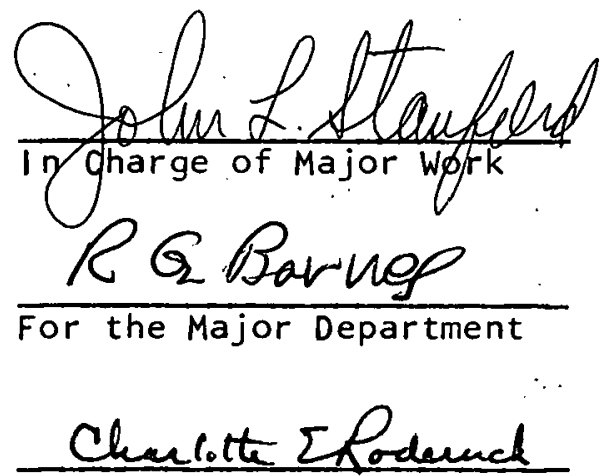

For the Graduate College

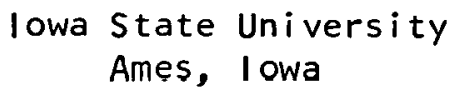




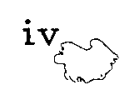

TABLE OF CONTENTS

\author{
ABSTRACT \\ 1. INTRODUCTION
}

$\underset{\mathrm{v}}{\text { Page }}$

1

11. EXPERIMENT 8

A. Impulsive Field Method 8

B. Sample Preparation 12.

C. Method of Obtaining Data 14

D. Frequency Results 19

III. DISCUSSION OF THE RESULTS . 32

A. General Considerations 32

B. Hole Octahedron at $\mathrm{H} \quad 36$

C. Hole Ellipsoids at $N$. 43

D. Electron Lenses at $\Delta \quad 47$

E. Electron Jack at $\Gamma$. 51

IV. CONCLUSIONS 58

$\begin{array}{ll}\text { V. BIBLIOGRAPHY } & 60\end{array}$

VI. ACKNOWLEDGEMENTS $\quad 62$

VII. APPENDIX A - COEFFICIENTS FROM INVERSION SCHEME FITS 63

VIII. APPENDIX B - FREQUENCY ANALYSIS PROGRAM LISTING AND DISCUSSION 


\section{Determination of the Fermi surface of molybdenum using the de Haas-van Alphen effect}

James Alvin Hoekstra

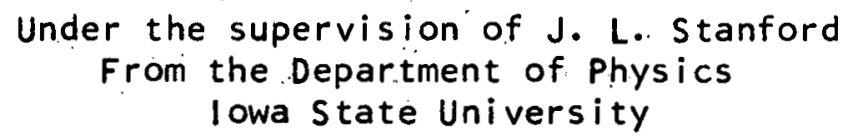

The de Haas-van Alphen effect in molybdenum has been studied in detail using large impulsive magnetic fields. Digital data recording, fast-Fourier frequency analysis by computer, and dynamic calibration of the entire apparatus have been used to obtain the de Haas-van Alphen frequencies to a high degree of accuracy. Several new frequency branches associated with the electron jack piece of the Fermi surface are reported. Frequency branches exhibiting high angular accuracy and resolution are reported for all branches that have previously been detected by other de Hass-van Alphen investigators. A quantitative description of all sheets of the Fermi surface is presented, along with comparisons with other Fermi surface radii obtained by use of the RFSE. The Mueller inversion technique for obtaining Fermi surface radi $i$ from the de Haas-van Alphen area data was used whenever possible. The de Haas-van Alphen results presented are consistent with an estimate of $2.5 \%$ of the $I H$ dimension for the size of the gap between the jack and the octahedral Fermi surface pieces. Thus the question about the size of this gap as raised by discrepancies greater than the combined experimental errors existing in

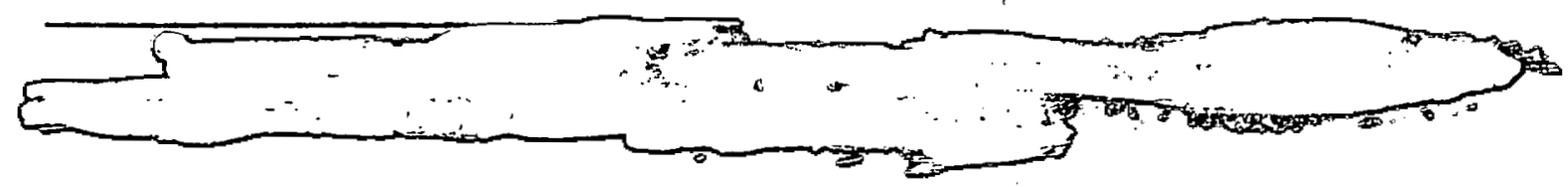




$$
\text { vi }
$$

current RFSE data is resolved. The spin-orbit parameter is not found to be anomalously higher than that for tungsten. 


\section{Silir \\ LIST OF. FIGURES}

Page

Figure 1. Brillouin zone for body-centered-cubic crystal

lattice

Figure 2. A $\langle 100\rangle$ cross section for the Fermi surface of molybdenum as proposed by Lomer (3)

Figure 3. A $\langle 110\rangle$ cross section for the Fermi surface of molybdenum as proposed by Lomer (3)

Figure 4. Block diagram of the experimental apparatus

Figure 5. Rotating sample holder. The drive wheel assembly is fitted over the drive shaft and plugged into the Amphenol connector

Figure 6. Variations of the fundamental de Haas-van Alphen frequencies in molybdenum for field directions in the $(001)$ and $(110)$ planes. The geometric axes for the rod shaped samples are: $\Delta,[110]$ and 0 , $[001]$

Figure 7. Linear plot of low frequency de Haas-van Alphen data for field directions in the (001) and (1T0) planes

Figure 8. Linear plot of medium frequency de Haas-van Alphen data for field directions in the (00I) and ( 110$)$ planes

Figure 9. Linear plot of high frequency de Haas-van Alphen data for field directions in the (001) and (1 i 0$)$ planes

Figure 10. Linear plot of low and medium frequency de Haasvan Alphen data for field directions in the (1ii) plane. Note the break in scale for $F(M G)$

Figure 11. Linear plot of high frequency de Haas-van Alphen data for field directions in the (IIII) plane

Figure 12. Comparison of the de Haas-van Alphen frequencies of molybdenum and tungsten. The solid lines indicate molybdenum data from this study. The dashed lines indicate tungsten data from Reference 22 


\section{viii}

Figure 13. Plots of the normalized frequency difference (in percent) $\Delta F=100$ ( $F_{\text {inversion }}-F_{\text {data }}$ )/ $F_{\text {data }}$ as a function of angle and $l$ value

Figure 14. Plots of the radii differences (in percent) $\Delta R=100\left(R_{l}=20,22\right.$ or $24^{-R_{l}}=18^{/ R_{l}}=18$ as a function of angle

Figure 15. Cross sections of the hole octahedron Fermi surface at $H$ for the $(001)$ and $(110)$ planes. The RFSE data of Boiko et al. (18) has been plotted directly, while the RFSE data of Cleveland and Stanford (20) has been multiplied by 1.05 before plotting

Figure 16. Cross sections of the hole octahedron Fermi surface at $H$ for the $(1 \overline{1} 1)$ and $(1 \bar{i} 2)$ planes

Figure 17. Cross sections of the hole ellipsoid Fermi surface at $N$ for the planes containing the three semiaxes. These directions are $\mathrm{N} \Gamma, \mathrm{NH}$, and NP

Figure 18. Cross sections of the electron Iens Fermi surface at $\Delta$ assuming a higher $D_{4 h}$ symmetry. For the nearly circular cross: section, the plane has normal direction $\Gamma \mathrm{H}$, and $0^{\circ}$ and $90^{\circ}$ are parallel to $\langle 100\rangle$ directions

Figure 19. (170) plane cross section for the electron jack Fermi surface at $\Gamma$. The solid and open circle data points and their labels are RFSE results of Boiko et al. (18). The + data point at $19^{\circ}$ from [001] and the solid line construction are deseribed in the text

Figure 20. (001) plane cross section for the electron jack Fermi surface at $\Gamma$. The open circle is a data point from Reference 18 . The + data points and the solid line construction are described in the text

Figure 21. A possible (iii) plane cross section for the electron jack Fermi surface at $\Gamma$. The open circles are data points from Reference 18 


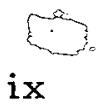

Figure 22. A plot of typical dHvA data. This data was taken at $28.9^{\circ}$ from [010] in the (001) plane

Figure 23. A filter-periodogram frequency analysis of the dHvi data shown in Figure 22

Figure 24. A fast-Fourier frequency analysis of the dHva data shown in Figure 22. The fundamental frequencies from this analysis are plotted and labelled in Figure 6 at $28.9^{\circ}$ from $[010]$ in the $(001)$ plane 


\section{$\times 3$}

\section{LIST OF TABLES}

Page

Table I. Experimental values for the dHvA frequencies. (in megagauss) corresponding to extremal crosssectional areas of the Fermi surface of Mo

Table 2. Radii for the hole octahedra in units of $(2 \pi / a)$

Table 3. Values in $\AA^{-1}$ for the semi-axes of the hole ellipsoids at $\mathrm{N}$

Table 4. Values in $\AA^{-1}$ for the radi $i$ of the electron lenses at $\Delta$ 


\section{INTRODUCTION}

The Fermi surface (FS) and the electronic structure of molybdenum have been the subjects of several extensive investigations over the past few years. Most of the results have been interpreted in terms of revisions of a model for the chromium group metals ( $\mathrm{Cr}$, Mo, W) first proposed by Lomer (1), who used the energy bands for Fe as calculated by Wood (2). The initial model was later corrected for the particular case of molybdenum (3). Theoretical augmented-plane-wave (APW) calculations for Mo done by Loucks (4) exhibit qualitative agreement with the Lomer model, but the quantitative results are subject to numerical errors due to an incorrect size for the unit cell used in determining the potential applied in the calculation (5).

The rhombic dodecahedron which defines the Brillouin zone, shown in Figure 1 , is labelled in accordance with the standard notation (6). As can be seen from the Lomer model in Figures 2 and 3 , the FS consists of several distinct pieces. The largest two are the hole "octahedron"s situated at the symmetry point, $H$ and the electron "jack" located at $\Gamma$. The "jack" consists of an octahedrally-shaped "body" with a "ball" protruding from each of the six corners of the "body." The narrow region connecting the "body" with one of the "balls" is referred to as a "neck." A hole "ellipsoid" is located at each of the symmetry points $N$. Six small electron "lenses" are situated along the lines $\Gamma H$ in the "neck" regions of the "jack." In the remainder of this paper the terms used to describe these FS pieces will no longer be enclosed in quotation marks. 


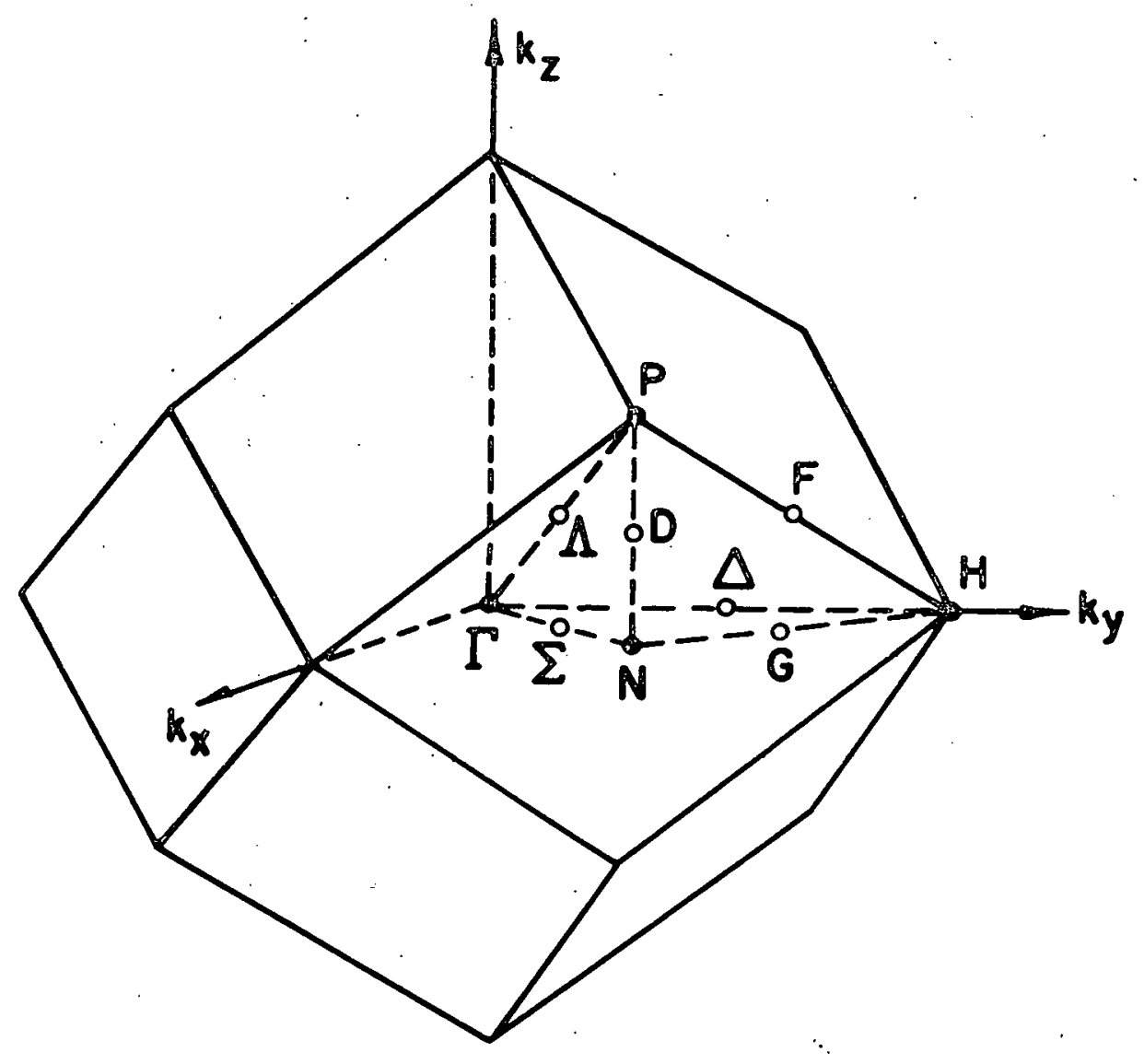

Figure 1. Brillouin zone for body-centered-cubic crystal lattice 


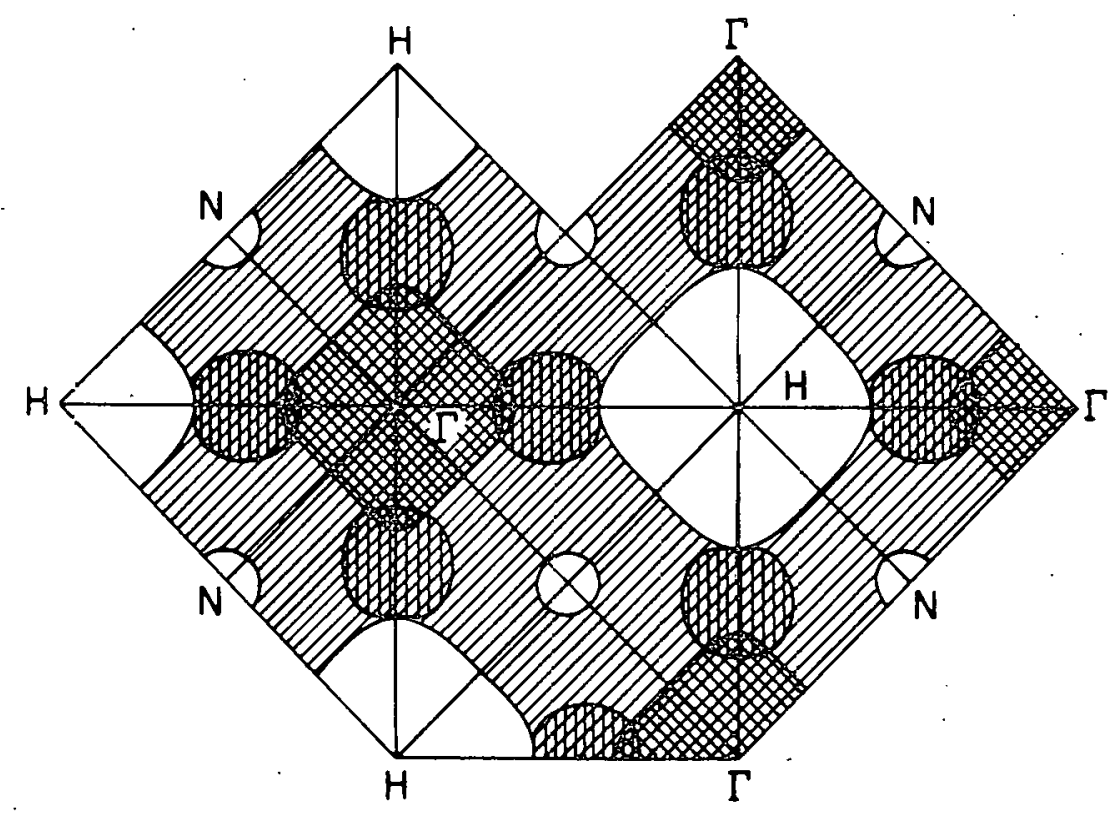

Figure 2. A $\langle 100\rangle$ cross section for the Fermi surface of molybdenum as proposed by Lomer (3) 


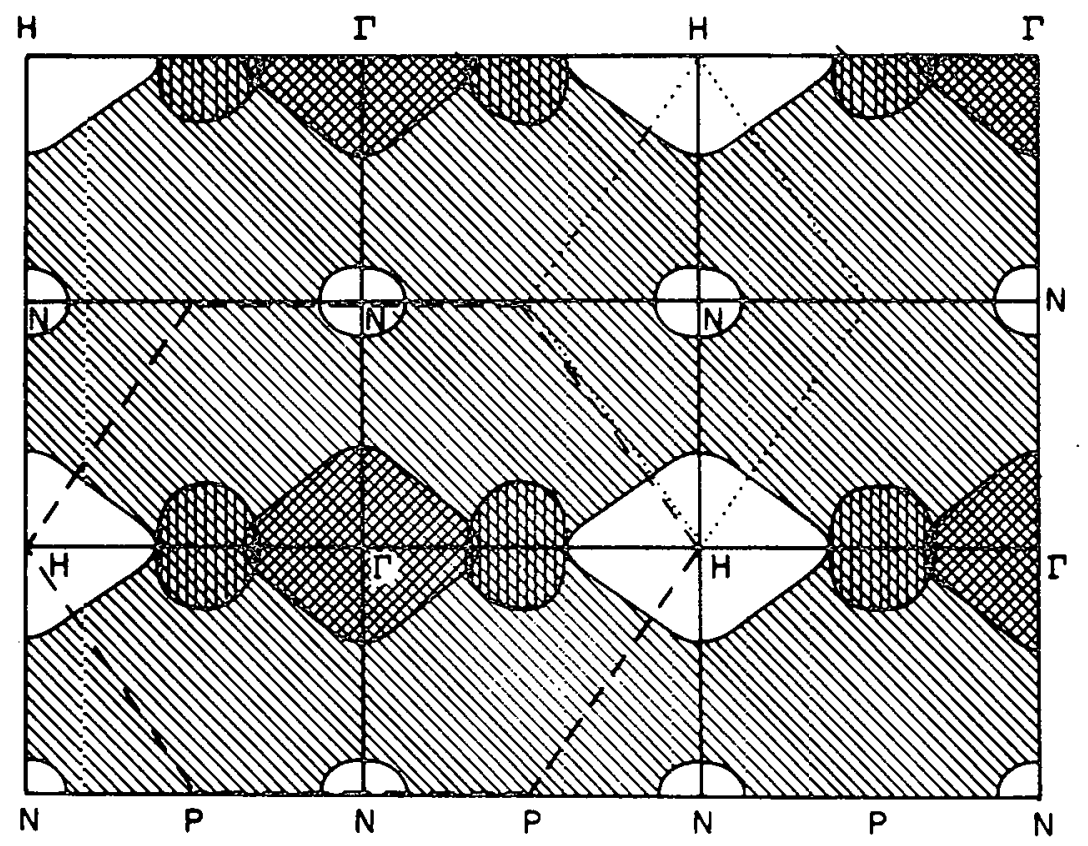

Figure 3. A $\langle 1 \mid 0\rangle$ cross section for the Fermi surface of molybdenum as proposed by Lomer (3) 
On the experimental side, the magnetoresistance measurements of Fawcett (7), of Fawcett and Reed (8), and of Alekseevskii, Egorov, Karstens, and Kazak (9) have shown that Mo is a compensated metal having equal numbers of electrons and holes. This means that the volume of the electron and the hole portions of the FS will be equal. Fawcett and Reed have shown there can be no more than about $10^{-4}$ open orbits per atom in Mo, which means that all sheets of the FS are simply-connected.

The anomalous skin effect measurements of Fawcett and Griffiths (10) were able to show that the FS area for a member of the $\mathrm{Cr}$ group transition metals is much less than the area of a sphere containing six electrons per atom. This suggests that the free electron model is a poor approximation to the Fermi surfaces of these metals.

Caliper dimensions along or near major symmetry directions were estimated by Jones and Rayne (II) and by BezuglyY, Zhevago, and Denisenko (12) in their magnetoacoustic effect investigations. Herrmann (13) has obtained the effective masses of several orbits in Mo and $w$ through the use of cyclotron resonance experiments. His results indicating a rather spherically shaped surface at $H$ disagree with the hole octahedron found at $\mathrm{H}$ by other investigators.

The first de Haas-van Alphen (dHvA) frequency results obtained by. using the torsion method with fields up to $18 \mathrm{~kg}$ on the $\mathrm{Cr}$ group metals were reported by Brandt and Rayne $(14,15)$. Their data for Mo came from orbits associated with the electron lenses. Sparlin and Marcus (16) presented dHvA torsion measurements on Mo and $W$ in fields up to $34 \mathrm{kG}$. Their data included information about the electron lenses, the hole 
ellipsoids, and partial information on the hole octahedra and the necks of the electron jack. No oscillations related to the body of the jack were observed. The pulsed-field dHvA results of Leaver and Myers (17) provide additional information about the jack and octahedron pieces, but their data exhibits a significant amount of scatter.

The recent radio-frequency size effect (RFSE) measurements of Boiko, Gasparov, and Guerdtsiteli (18), of Cleveland (19), and Cleveland and Stanford (20) provide many caliper dimensions of the FS but the data presented in these two investigations differ by $6 \%$, a discrepancy which is greater than the combined experimental errors claimed by the investigators. The RFSE data is most helpful in defining the shapes of the larger FS pieces, but is hard to obtain and interpret for the ellipsoids and lenses, where the RFSE signals lie close together in magnetic field value, and are difficult to separate and follow as a function of angle. The separation or gap between the jack and the octahedral FS pieces is estimated by Boiko et al. as $2.5 \%$ of the $\Gamma H$ dimension, while cleveland's estimate for the gap is $7 \%$. The larger gap was explained by choosing a value of the spin-orbit parameter for Mo larger than that predicted by Mattheiss (21) for $W$. The effects of spin-orbit coupling should be more pronounced in $W$, where the gap is $5 \%(22)$, than in Mo.

This investigation of Mo was undertaken to provide complete and accurate data concerning the extremal areas associated with each of the pieces of the FS using the pulsed-field dHVA technique. The data will be used to obtain the FS radii and thus clarify the discrepancy existing in the current RFSE data, and to resolve the question about the size of the 
gap between the jack and the octahedron caused by the spin-orbit interaction. 


\section{EXPERIMENT}

\section{A. Impulsive Field Method}

The de Haas-van Alphen effect is observed as oscillations in the magnetization of pure metal single-crystal specimens at liquid-He temperatures in strong magnetic fields. Onsager (23) worked out a simple theory giving the frequency of dHvA oscillations by applying the BohrSommerfeld quantization rules to the motions of electrons in an applied magnetic field. The motion of electrons in k-space normal to the applied magnetic field is quantized into orbits which enclose areas proportional to the field. As the field is increased, these orbits cross the Fermi surface, depopulate, and therefore induce oscillations in the free energy and the magnetic moment of the system. The frequency $F$ for these dHvA oscillations is

$$
F=\frac{C \hbar}{2 \pi e} A_{O}\left(E_{F}\right)=104.7\left(\frac{M G}{\AA^{-2}}\right) A_{O}\left(E_{F}\right)
$$

where $A_{O}\left(E_{F}\right)$ is an extremal cross-sectional area of the Fermi surface normal to the applied field. Experimental observations have shown that the dHvA oscillations are actually periodic in $1 / B$ rather than $1 / H$. Since the real space electron orbits have diameters extending over hundreds of lattice spacings, it is reasonable that the electrons should see an average field $\underline{B}$ inside the sample rather than the externally applied field $\underline{H}$.

A detailed calculation of the amplitude dependence of the dHvA effect was made by Lifshitz and Kosevich (24), who applied statistical 
mechanics to a system of independent electrons in an applied magnetic. field. Dingle (25) has shown that if the effect of electron scattering due to collisions is included, the $r^{\text {th }}$ harmonic term is multiplied by a factor $K$ which is

$$
K=\exp \left(-2 \pi^{2} r m^{*} k_{B} T_{D} / e \hbar H\right)
$$

where $T_{D}$ is an effective temperature, the Dingle temperature, related to the width of the Landau levels. This term is very important in drastically reducing the total signal strength of the dHvA effect in samples which are not pure or of good crystal perfection. Such is not the case with our samples.

The effect of conducting a dHvA experiment at a finite temperature is to cause thermal damping of the oscillations. This thermal damping factor $I_{r}$ is given for the $r^{\text {th }}$ harmonic by

$$
i_{r}=\frac{x_{r}}{\sinh x_{r}}=2 x_{r} e^{-x_{r}}\left[1+e^{-2 x_{r}}+\cdots \cdot\right]
$$

where,

$$
x_{r}=2 \pi r^{2} \frac{k_{B} T}{\pi_{c}}=2 \pi^{2} r \frac{m^{*} k_{B} T}{e H H}
$$

$x_{r} \doteq 3 r$ for free electrons in a field of $50 \mathrm{~kg}$ and at a temperature of

I K. A recent article by Gold (26) includes this expression and provides a comprehensive review of the theoretical aspects of the dHVA effect and the additional experimental techniques possible.

In this study dHvA oscillations in Mo were observed using the impulsive field method developed by Shoenberg (27) in 1957. Since the basic 
impulsive field apparatus was essentially the same as that used by Anderson (28), and Panousis (29), only a brief summary will be included here.

A single-crystal specimen of high-purity material was immersed in a dewar of liquid $\mathrm{He}$, which was designed so that the sample was situated inside the center of a solenoid. This entire assembly was contained in a dewar of liquid $\mathrm{N}_{2}$ for cooling. A block diagram of the experimental circuitry is shown in Figure 4. A bank of capacitors with $5040 \mathrm{mfd}$ total capacitance was charged to a maximum of 2400 volts. Discharging these capacitors through the solenoid produced a peak field of about 180 KG with a pulse time of $21 \mathrm{~ms}$. This time-varying magnetic field induced a voltage proportional to $d M / d t=(d M / d H) /(d H / d t)$ in a pickup coil surrounding the sample. The magnetic field values were determined from the voltage readings across a $0.01 \Omega$ standard resistor. Both the field values and the pickup coil voltage were sometimes monitored through the use of a dual-beam oscilloscope and Polaroid pictures taken of this output for permanent pictorial data storage. The pickup coil voltage was also displayed on a Hughes Model 104 Memo-scope storage oscilloscope as a monitor of signal quality and strength for each pulse. Both the pickup coil voltage and the voltage from the standard resistor were appropriately amplified, converted from analog to digital signals and stored in a small memory for later readout onto punched paper tape.

The pickup coil consisted of 2000 turns of No. 50 insulated copper wire followed by $1 \frac{1}{2}$ layers of cigarette paper and about 860 turns wound in a reverse manner. The final number of reverse turns was adjusted so 


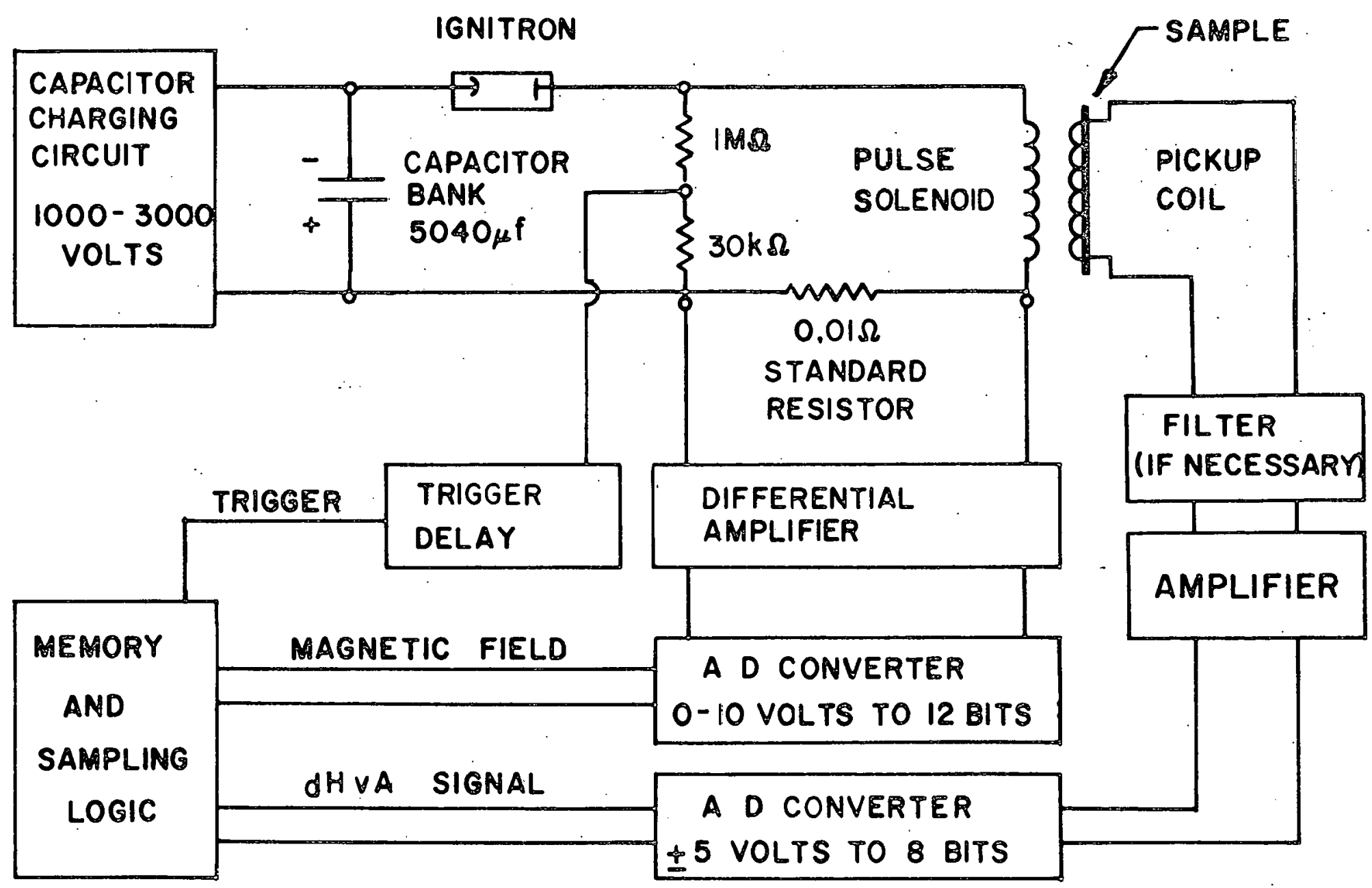

Figure 4. Block diagram of the experimental apparatus 
that the "bucked" coil would not be able to detect the uniform field. produced by the pulse solenoid, but would be able to pick up the changes in the magnetization of the sample residing inside. Sinceit is difficult to determine the exact number of turns necessary to completely "buck" the uniform time-varying field, a few turns of wire connected to an additional pair of leads from the top of the sample holder provided a signal which could be used to eliminate most of the remaining $24 \mathrm{~Hz}$ signal from the main field. These turns will be referred to later as the "bucking coil." Otherwise the dHVA signal appears as a ripple riding on an approximate one-half sine wave pulse coming from the solenoid.

\section{B. Sample Preparation}

The single-crystal samples used in this investigation were spark-cut from the same single-crystal rod of Mo from which Cleveland obtained samples for his. RFSE experiment (19). The rod, purchased from Westinghouse Lamp Division, has a residual resistance ratio $\left(R_{300 K} / R_{4.2 K}\right)$ of 5000 as determined by use of the eddy-current decay method (30). Two samples for this investigation were cut in the form of long, thin square bars -one with a $\langle 100\rangle$ crystallographic axis parallel to the length of the bar and the other with a $\langle 110\rangle$ direction as the long axis. These samples were electro-polished to a diameter of $0.5 \mathrm{~mm}$ and a length of $5 \mathrm{~mm}$, using a $6 \%$ solution of perchloric acid in methanol cooled with, a mixture of dry ice and acetone.

A sample was mounted in a pickup coil housed in the drive wheel of the sample holder designed by Panousis and shown in Figure 5. The drivewheel assembly was detached from the drive-shaft assembly by disconnecting 


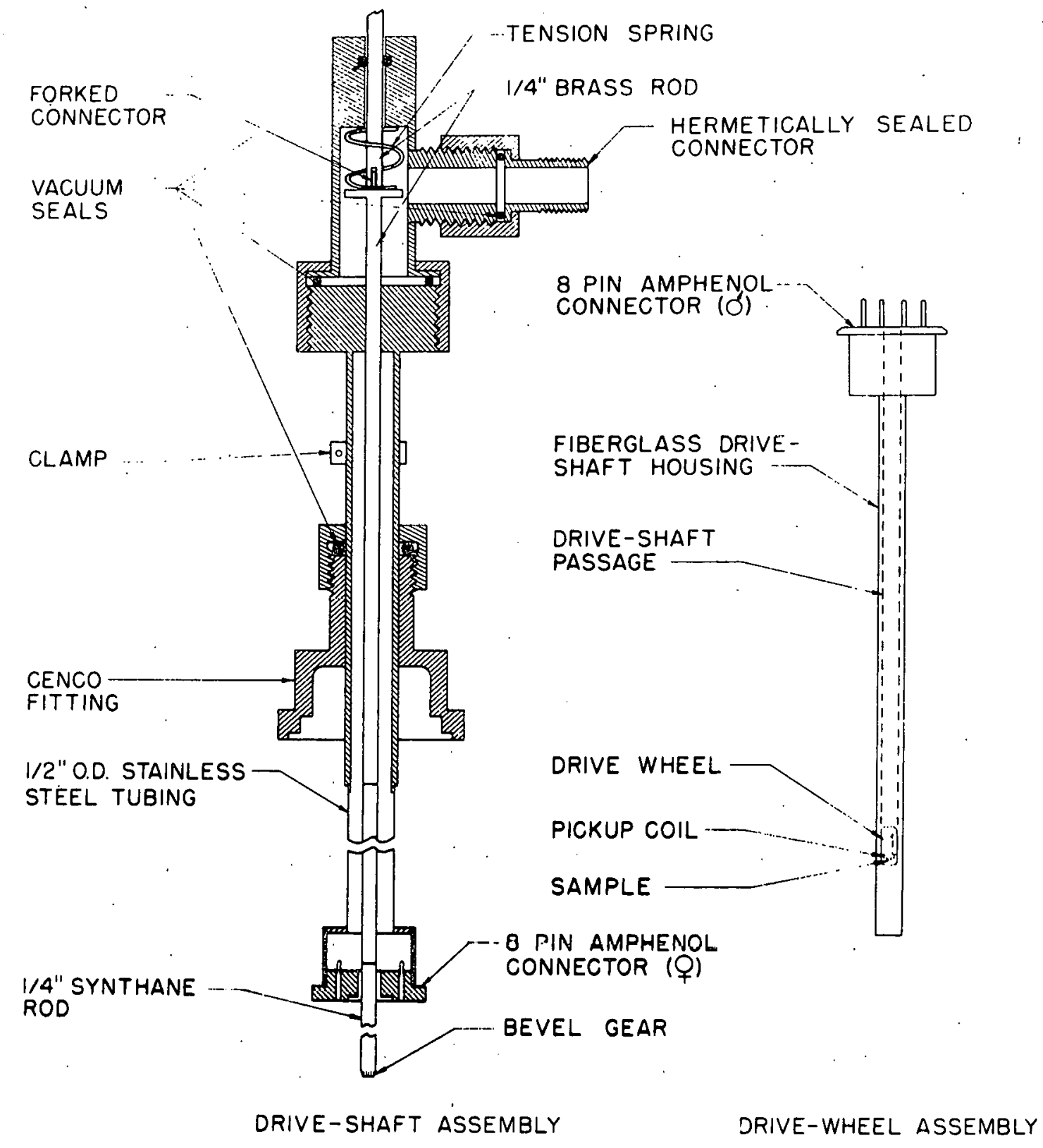

Figure 5. Rotating sample holder. The drive wheel assembly is fitted over the drive shaft and plugged into the Amphenol connector 
the 8-pin Amphenol connectors and was mounted in a jig capable of keeping the plane of rotation of the drive wheel parallel to a Polaroid $x$-ray camera used for checking the orientation of the sample. The x-ray beam entered a small hole in the wheel parallel to its axle and struck the tip of the sample which slightly protruded out of the end of the pickup coil. The Laue back-scatter diffraction pattern obtained enabled us to determine the plane of rotation of the sample to within $7^{\circ}$ of a chosen crystallographic direction.

\section{Method of Obtaining Data}

To obtain all of the frequency data possible from the sample, one would like to have some way of being able to discriminate against some frequencies while enhancing others. Then a higher amplitude signal from a given set of frequencies would enable a more accurate determination of the frequencies involved. Such discrimination is possible by exploiting the effects of the thermal damping term expressed in Equation 3. One data run for each orientation plane with a given sample was made at a temperature of $4.2 \mathrm{~K}$ using a peak field of $90 \mathrm{kG}$. This mode of operation enhanced the amplitudes of frequencies arising from the lenses and the ellipsoids. A second data run for each plane and sample was made at $1.2 \mathrm{~K}$ using a peak field of $170 \mathrm{kG}$. At this temperature and higher field the frequencies associated with the larger and/or higher effective mass orbits arising from the electron jack and the hole octahedron were brought out.

To contribute to the dHvA effect, electrons must complete one or several orbits in the real space of the crystal lattice. The size of the orbit is inversely related to the magnetic field strength. As the 
magnetic field increases, more possible orbits begin having path lengths on the order of the mean free path $\lambda$, which is dictated by the sample purity as well as the temperature. Electrons which can complete an orbit contribute to the dHvA effect rather than being randomly scattered. The size of the orbit also depends upon the possible momentum states for the given electron as dictated by its position very near the FS in momentum space, and the direction of the applied magnetic field. Increased temperature causes the FS to become less sharply defined and increases the probability that the electron will be scattered onto a new path. The effective mass is a measure of the curvature of the particular orbit, and tells how easily the electron will be able to traverse the path that it is prescribed to follow. Temperature, effective mass and field strength all appear in the thermal damping term.

At $4.2 \mathrm{~K}$ and $90 \mathrm{kG}$ peak field the total signal strength from the pickup coil was such that a gain of around 500 was needed to provide the \pm 5 volts maximum signal to the analog-to-digital converter $(A D C)$. A solid-state amplifier built by the Ames Laboratory Instrumentation Group especially for the dHvA investigation in lutetium by this author was satisfactory for this signal level. This amplifier, giving linear response from $1 \mathrm{kHz}-100 \mathrm{kHz}$, provided a low-noise output except near its maximum gain of 50,000. Although the amplifier was non-linear at $24 \mathrm{~Hz}$, the signal at this frequency due to the pulse solenoid was rejected by using the "bucking coil" mentioned in Section $\| 1-A$. The "bucking coil" signal was reduced by a Dekatran transformer and fed to the first-stage differential amplifier in such a manner as to subtract out most of the 
remaining pulse solenoid field which was detected along with the desired dHvA signal by the pickup coil, which was the other input to the same differential amplifier. Through the use of this complete amplifier we have provided a good wide-band dHvA signal of \pm 5 volts maximum as input to the $A D C$ without using any electronic or static filtering which could introduce noise.

At $1.2 \mathrm{~K}$ and the higher peak field the increased signal strength overloaded the stages ahead of the gain control on the amplifier mentioned above, so an older system was substituted. Here the pickup coil signal was the input to a Krohn-Hite Model $315 A R$ electronic filter set with a band-pass of $5 \mathrm{kHz}$ to $100 \mathrm{kHz}$. The filter was used to eliminate the 24 $\mathrm{Hz}$ pulse field signal and the associated "ringing" of the Infrared Model 603 amplifier which followed the filter in the circuit. This amplifier, which had a poorer frequency response than the newer one, was sufficient to provide a gain of around 80 with a reasonably good signal for the ADC.

For each plane of orientation of a given sample with respect to the magnetic field, and the temperature and peak field combinations mentioned above, data was obtained at $2^{\circ}$ intervals as indicated by a counter dial connected by a set of gears to the $1 / 4^{\prime \prime}$ brass rod at the top of the sample holder shown in Figure 5. Backlash in the gears was eliminated by always advancing the counter to increasing angles from the beginning of a particular data run. Although the approximate symmetry direction of the sample for a certain dial reading was known and used to determine the angular interval over which data was taken, the final choice of the symmetry direction was made from the symmetry of the plotted frequency information 
about some particular angle.

At each particular angular setting the solenoid was pulsed four times at $3 \mathrm{~min}$. intervals for low peak field or 5 min. intervals for high peak field to allow the solenoid to reach thermal equilibrium. During two of these pulses the trigger delay was set so that the digital equipment would sample $8 \mathrm{msec}$ of data occurring before the peak field ("rising field") and during the other two pulses for data occurring after the peak field ("falling field"). Each such pulse produced a set of 2008 values for the dHvA signal and 20 precise values for the magnetic field sampled in equal increments of time with a field value taken once every 100 dHvA signal samplings. The digital information was dumped from a small memory onto punched paper tape. This instrumentation is described in Reference 31. Each data set was analyzed over a series of frequency ranges using the fast-Fourier transform program $I$ isted and discussed in Appendix $B$. A time constant $T$ of $40 \mu \mathrm{sec}$ was found appropriate to correct for the time delay introduced by the finite inductance of the "non-inductive" standard resistor, for eddy current effects, and for other effects, including electronics, capable of introducing a time delay. The true field $H$ is related to the apparent (measured) field $H^{\prime}$ (see Reference 31 ) by

$$
H=H^{\prime}-\tau(d H / d t) \quad .
$$

This correction produced dHvA results that were the same for both "rising field" and "falling field" data. Corresponding frequencies from each of the four data sets obtained at a given angle were averaged together to produce a single value which could be plotted on graphs of the data. 
The number of degrees between symmetry points of the plotted raw data disagreed slightly with the dial readings of the sample holder. Since the $\frac{15}{4}$ synthane rod of the sample holder (Figure 5) had a 14 tooth bevel gear which meshed into the 36 teeth of the drive wheel, the small number of teeth (which were cut as precisely as possible) could be a source of error in knowing the angle at which the data was taken. To correct for this error, the following procedure was adopted. The pulse solenoid was driven by a low-frequency oscillator run at maximum power. A lock-in detector. and digital voltmeter measured the pickup coil voltage as a function of angle determined by the dial reading. The resulting cosinelike dependence of the voltage of the pickup coil in a uniform magnetic field was plotted along with a perfect cosine wave. The engaging of individual teeth as the angle was changed was visible on the plot. A table of actual angle for a given dial reading was constructed and these angle corrections applied to all the data. Such corrections are meaningful only if the shaft engaged the wheel at the same place for a given dial setting each time the sample holder was assembled, which was the case for this entire investigation.

Pulse solenoid G, used for all of the data presented here, was calibrated using the dHvA effect itself to make a dynamic calibration of the entire apparatus. The [111] frequency of the $\nu$ oscillation in tungsten, determined by O'Sullivan and Schirber (32) to be $98.8 \pm 0.2 \mathrm{MG}$ with the aid of NMR measurements of quasi-static fields, was used as the frequency standard. The field constant determined in this way was

$$
K=135.68 \pm 0.3 \mathrm{G} / \mathrm{amp} .
$$


This constant can be compared with one determined by Panousis (29) for the same solenoid, using lead as a standard, to be $K=134.7 \pm 0.3 \mathrm{G} / \mathrm{amp}$ with a systematic error of up to $0.5 \%$ because of sample misalignment, and with the static calibration of the entire apparatus made earlier by Phillips (33) which yielded a constant $K=135.6 \mathrm{G} / \mathrm{amp}$. The static calibration and the dynamic calibration with tungsten agree within $0.1 \%$. The $3 / 4 \%$ discrepancy quoted in Reference 22 seems to have been resolved by this additional calibration run.

The experimental data which is presented in the following section was subjected to the following criteria: (1) The frequency presented should be the average of corresponding frequencies found in the four data sets taken at that angle. (2) The frequency should be a member of a set of frequencies which are smoothly varying in value and amplitude as a function of angle of observation. (3) Sets of frequencies which can be identified as 2 nd or 3 rd harmonics, or sums and differences of other reported frequencies are omitted.

\section{Frequency Results}

The angular dependence of the fundamental dHvA frequencies corresponding to extremal cross-sectional areas of the Fermi surface of Mo are shown in Figures 6 through 11 . In all of these figures the symbol $(\Delta)$ is used to represent data taken with the [110] sample while the symbol (0) is used for the [00I] sample. The labels for the frequency branches are those used by Girvan et al. (22), except for the $\beta$ used for lens frequencies, which do not exist in $W$. 


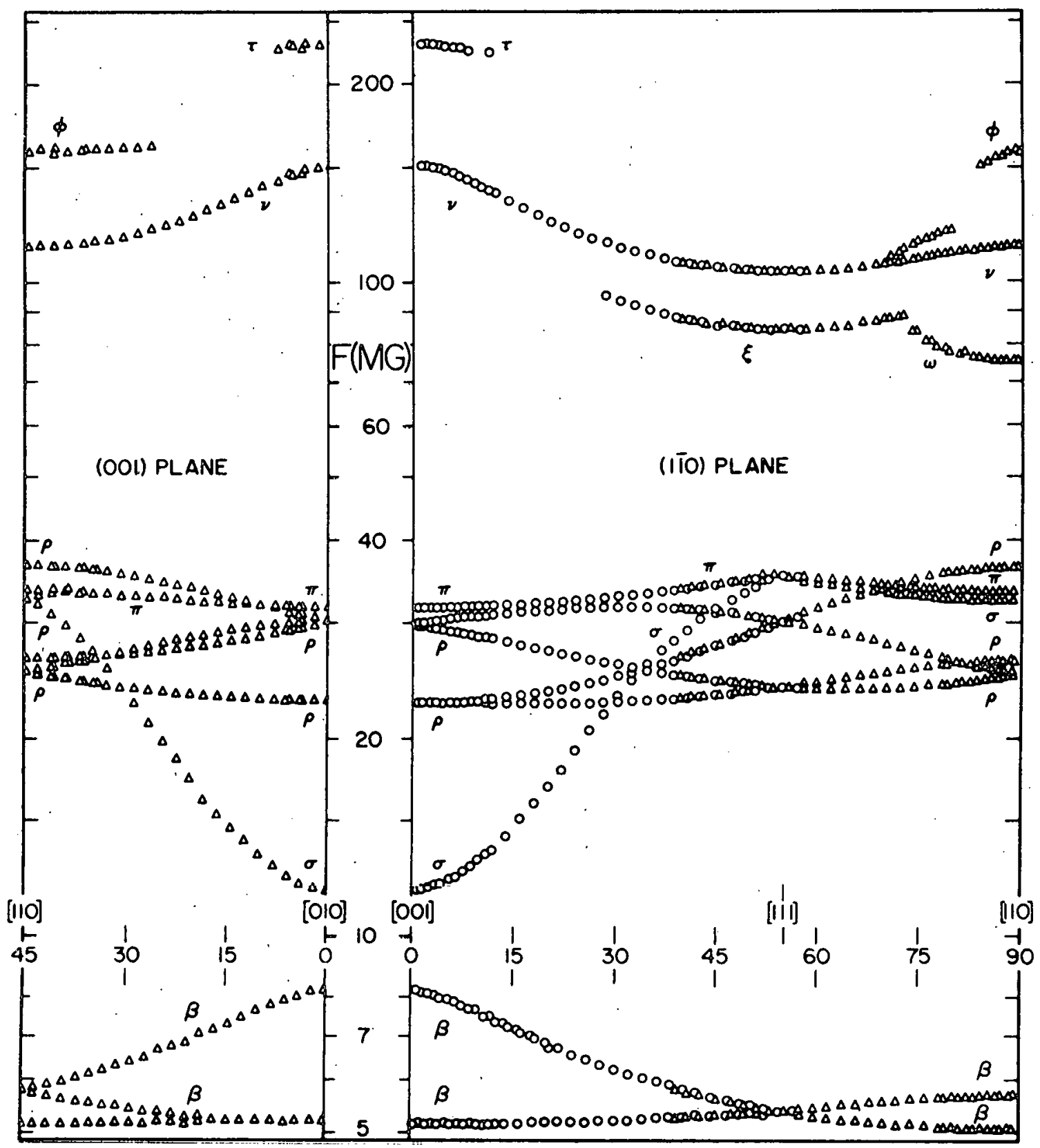

Figure 6. Variations of the fundamental de Haas-van Alphen frequencies in molybdenum for field directions in the (001) and ( 1 i 0 ) planes. The geometric axes for the rod shaped samples are: $\Delta,[110]$ and $0,[001]$ 


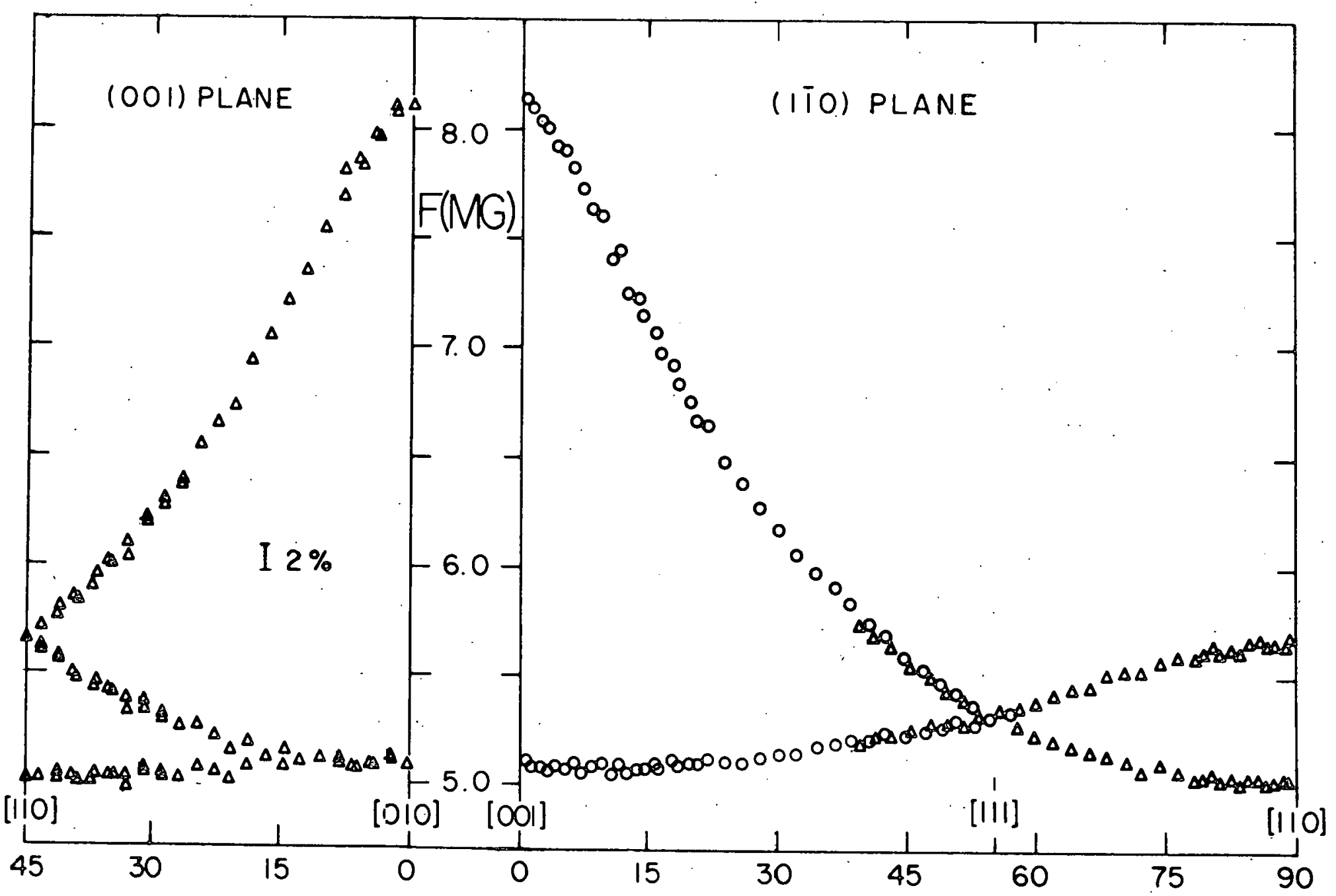

Figure 7. Linear plot of low frequency de Has-van Alphen data for field directions in the (001) and (ijo) planes 


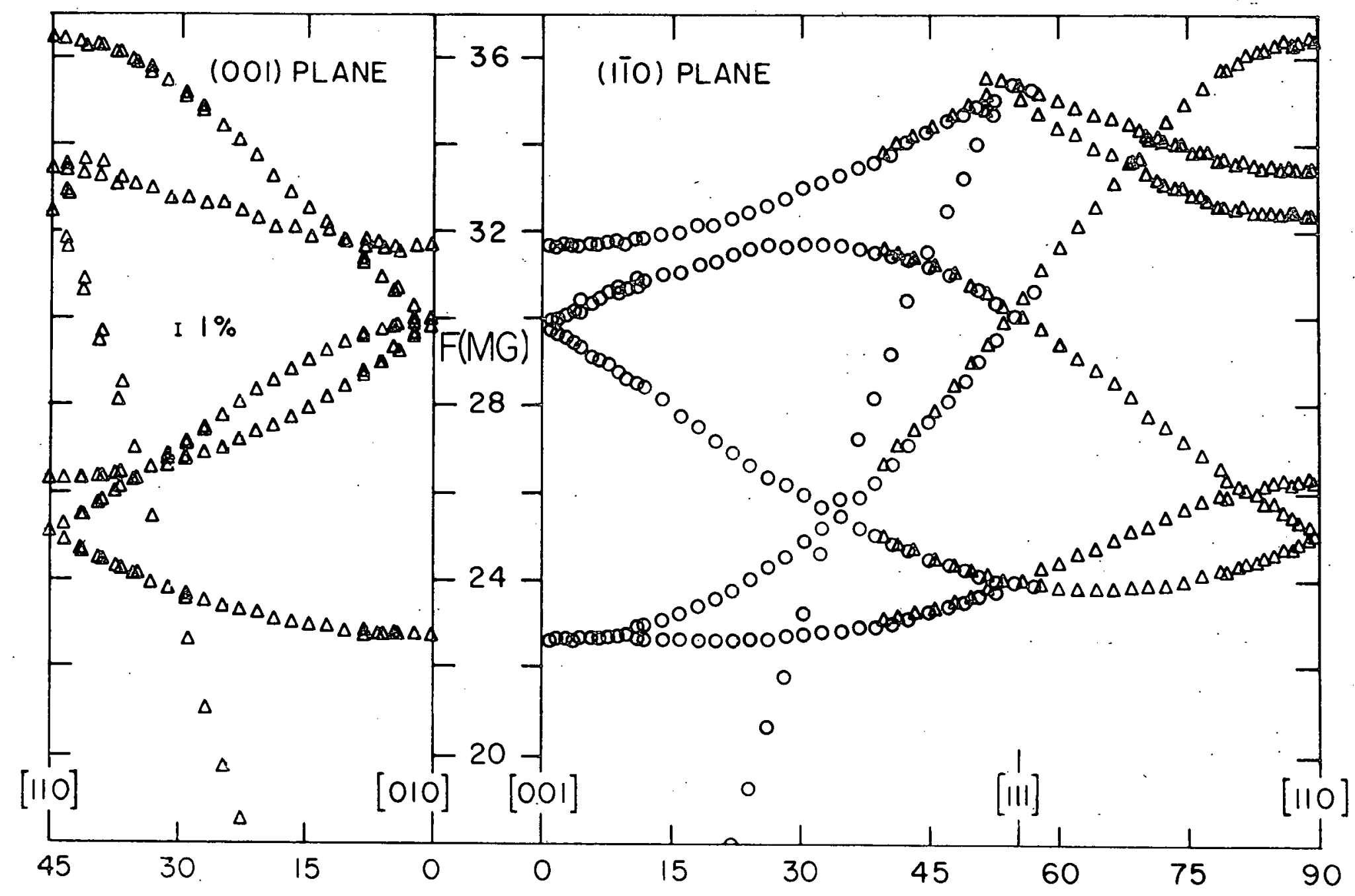

Figure 8. Li near plot of medium frequency de Haas-van Alphen data for field directions in the (0II) and ( 1 i 0 ) planes 


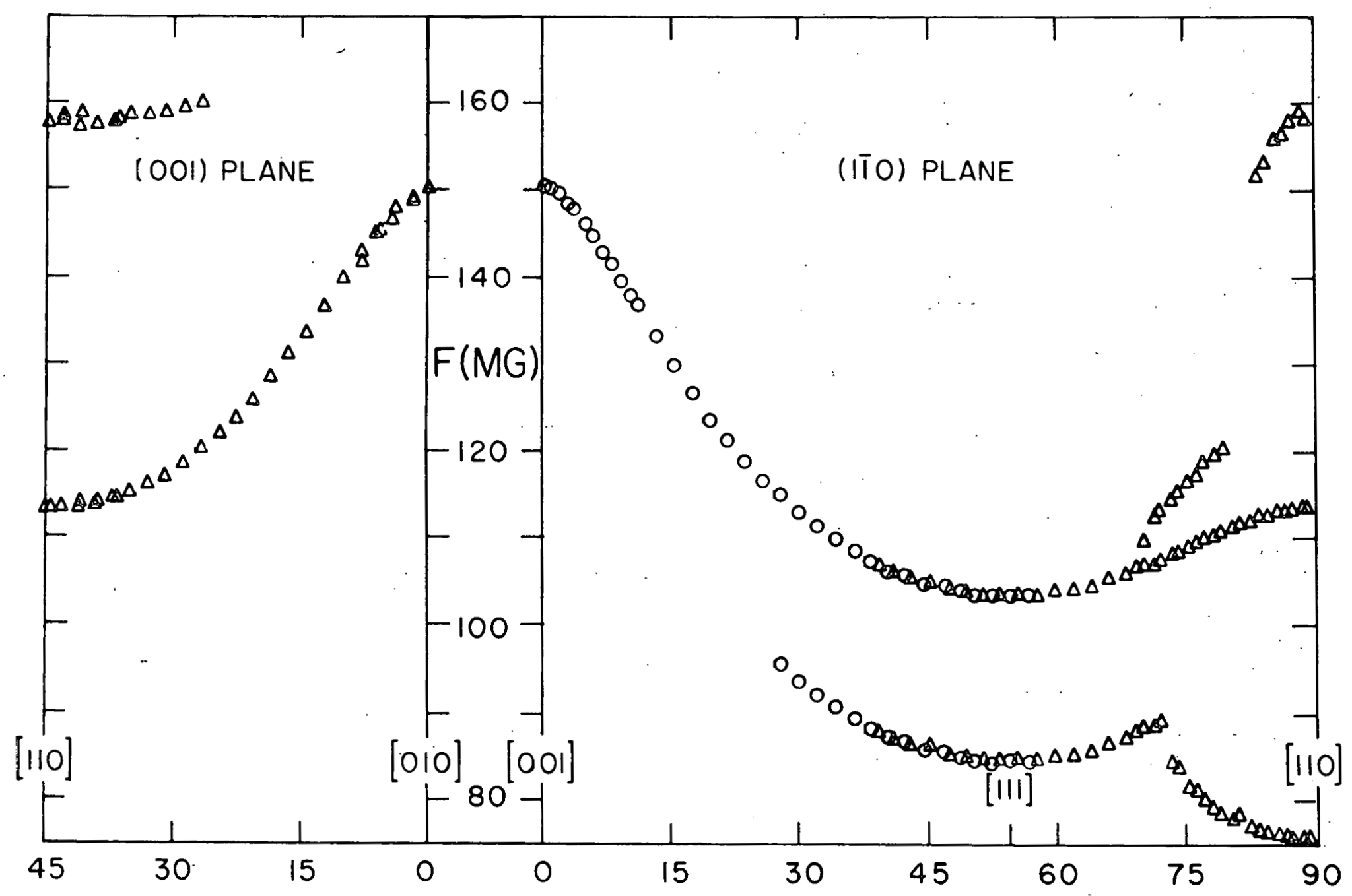

Figure 9 . Linear plot of high frequency de Haas-van Alphen data for field directions in the (00I) and (1i0) planes 


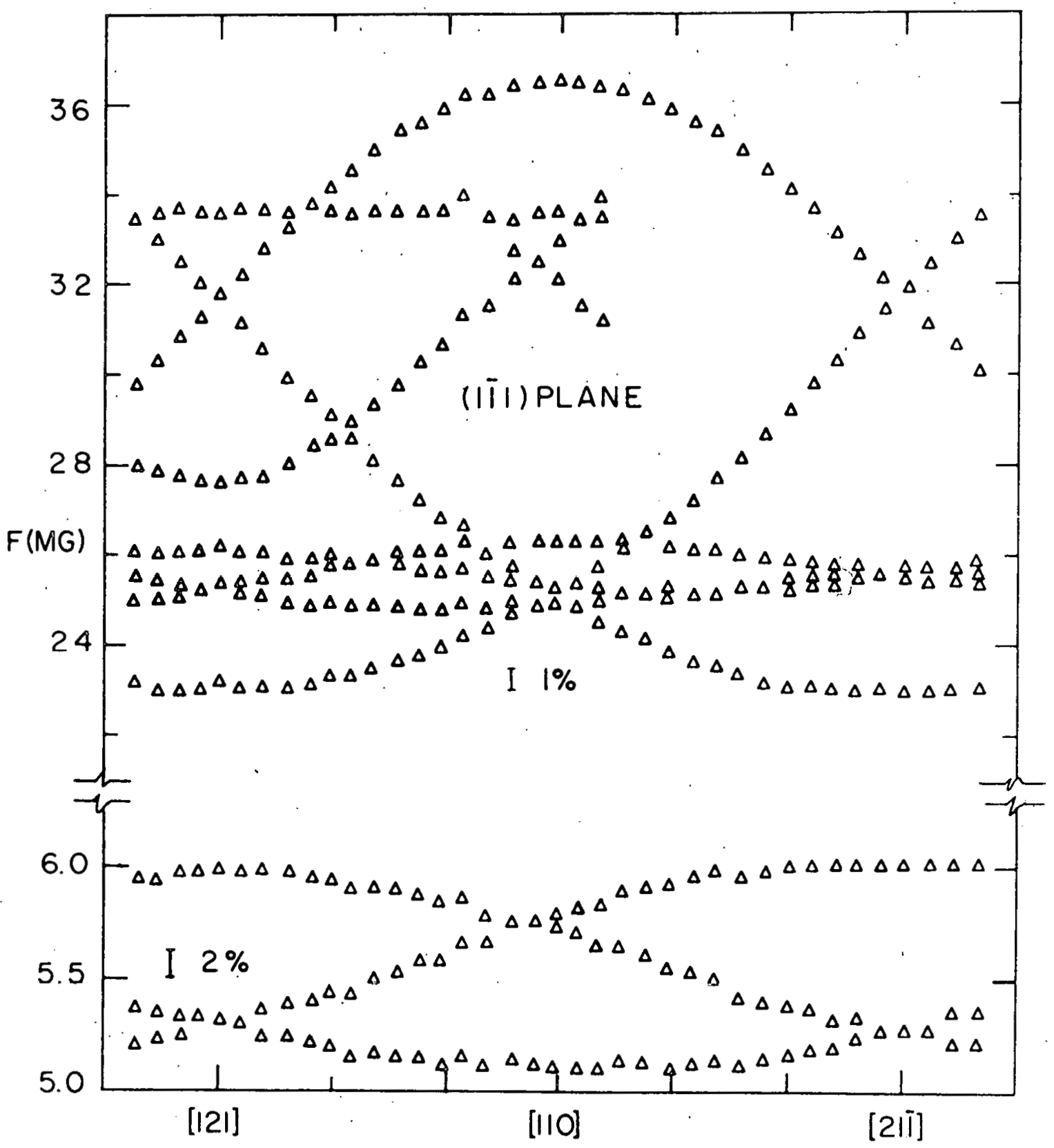

Figure 10. Linear plot of low and medium frequency de Haas-van Alphen data for field directions in the (1ii) plane. Note the break in scale for $F(M G)$ 


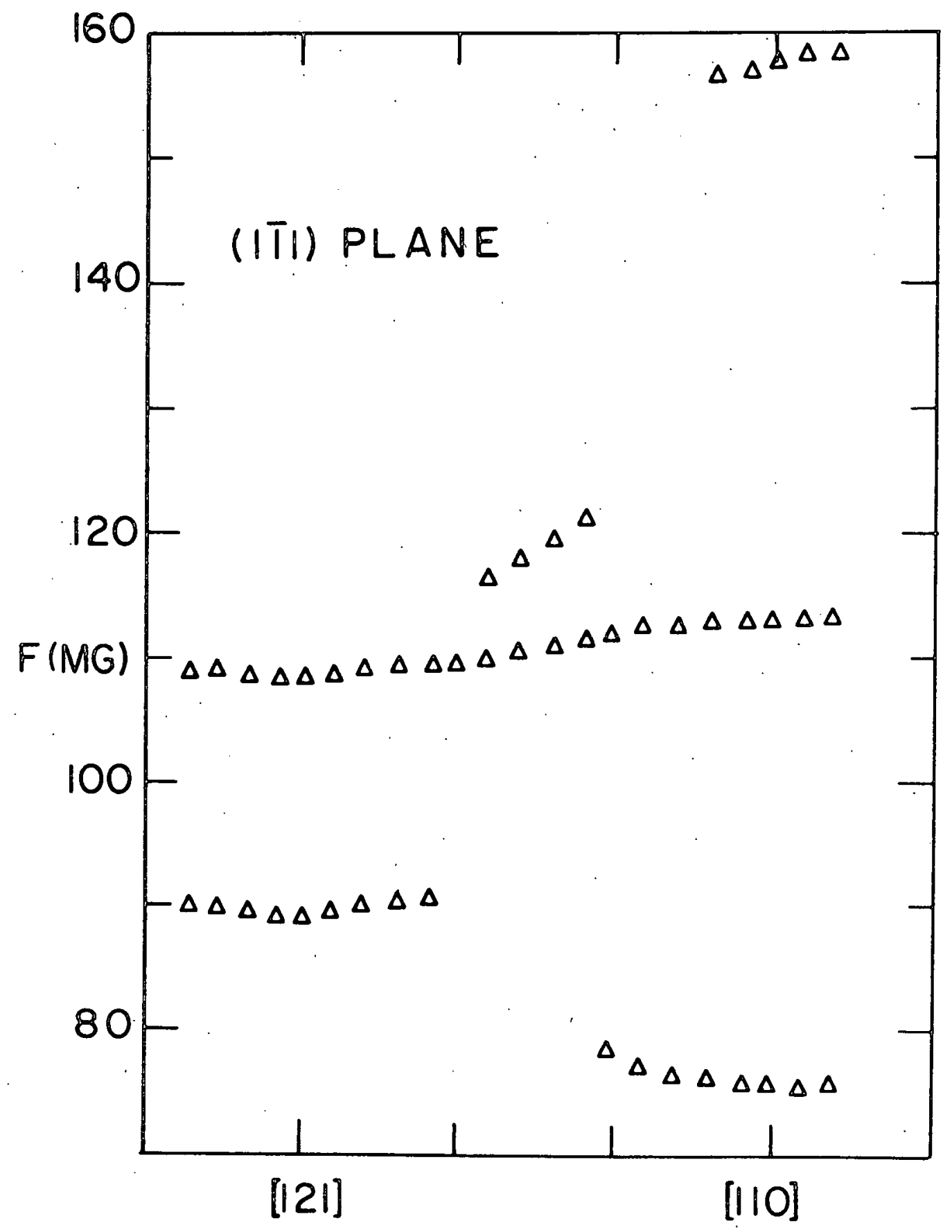

Figure 11. Linear plot of high frequency de Haas-van Alphen data for field directions in the ( 171 ) plane 
The only correction applied to all of the data was that for angle calibration as described in Section 1/-C. In Figures 6 through 9 a symmetry direction was chosen for the corrected data taken over an approximately $60^{\circ}$ range with a given sample and plane of orientation, and this data was folded about the chosen symmetry direction. Wherever the density of data as a function of angle is greater than one set of frequencies per approximate two degree interval, this folding process has taken place.

dHivA frequencies measured at a given symmetry direction should be identical, no matter which plane of orientation has been chosen or the sample being used. These frequencies, listed in Table l along with values determined by other experimentalists, can be compared for a given symmetry direction. Any discrepancies found are the result of misalignment of the sample in the plane of rotation, or the precision with which a given frequency can be determined (usually better than $\frac{1}{2} \%$, except for around $1 \%$ for low frequencies).

Figure 6 is a semi-logarithmic plot of all of the data obtained in the $(001)$ and $(1 \overline{0})$ planes. Figures 7,8 , and 9 are linear plots of most of the data appearing in Figure 6. The precision with which the samples were oriented can be seen from several aspects of this data. The folded data shows very little scatter as a function of angle near the [110] and [001] symmetry directions. The scatter in the folded data at [010] in the (001) plane is a function entirely of the choice of a symmetry direction at [110], since all of the information reported in the plane comes from the [110] sample. The scatter in the data appearing near [111] 
THIS PAGE

\section{WAS INTENTIONALLY \\ LEFT BLANK}


Table 1. Experimental values for the diva frequencies (in megagauss) corresponding to extremal cross-sectional areas of the Fermi surface of Mo

\begin{tabular}{|c|c|c|c|c|c|c|c|c|c|c|c|}
\hline \multicolumn{4}{|c|}{ (001) PLANE } & \multicolumn{4}{|c|}{ (1) } & \multicolumn{4}{|c|}{ (1וֹ) PLANE } \\
\hline Labe 1 & $\begin{array}{l}\text { Frequency } \\
\text { this } \\
\text { study }\end{array}$ & $\begin{array}{l}\text { Other } \\
\text { result } \\
\quad(15)\end{array}$ & $\begin{array}{l}\text { Other } \\
\text { result } \\
\quad(16)\end{array}$ & Lábel & $\begin{array}{c}\text { Frequency } \\
\text { this } \\
\text { study }\end{array}$ & $\begin{array}{l}\text { Other } \\
\text { result } \\
\text { (15) }\end{array}$ & $\begin{array}{l}\text { Other } \\
\text { result } \\
\text { (16) }\end{array}$ & Labe I & $\begin{array}{l}\text { Frequency } \\
\text { this } \\
\text { study }\end{array}$ & $\begin{array}{c}\text { Other } \\
\text { result } \\
(15)\end{array}$ & $\begin{array}{l}\text { Other } \\
\text { result } \\
(16)\end{array}$ \\
\hline \multicolumn{4}{|c|}{ [010] direction } & \multicolumn{4}{|c|}{$[001]$ direction } & & & & \\
\hline $\begin{array}{l}\beta \\
\beta \\
\sigma \\
\rho \\
\rho \\
\pi \\
\nu \\
T\end{array}$ & $\begin{array}{r}5.19 \pm .03 \\
8.21 \pm .04 \\
11.73 \pm .05 \\
22.7 \pm .1 \\
29.9 \pm .1 \\
31.65 \pm .1 \\
150.3 \pm .5 \\
231 \pm . \pm\end{array}$ & $\begin{array}{l}5.15 \\
7.92\end{array}$ & $\begin{array}{r}5.21 \\
8.23 \\
11.78 \\
23.0 \\
31.2\end{array}$ & $\begin{array}{l}\beta \\
\beta \\
\sigma \\
\rho \\
\rho \\
\pi \\
\nu \\
\tau\end{array}$ & $\begin{array}{r}5.18 \pm .03 \\
8.25 \pm .04 \\
1.73 \pm .05 \\
22.7 \pm .1 \\
29.9 \pm .1 \\
31.63 \pm .1 \\
150.5 \pm .5 \\
232 \pm 3\end{array}$ & $\begin{array}{l}5.17 \\
8.00\end{array}$ & $\begin{array}{r}5.33 \\
8.53 \\
12.30 \\
23.0 \\
31.1\end{array}$ & & . & $\cdot$ & \\
\hline \multicolumn{4}{|c|}{$[110]$ direction } & \multicolumn{4}{|c|}{$[110]$ direction } & \multicolumn{4}{|c|}{ [110] direction } \\
\hline $\begin{array}{ll}\beta & \\
\beta & \\
\rho & \\
\rho & \\
\sigma & \\
\pi & \\
\rho & \\
\omega & \\
\nu & 1 \\
\phi & 1\end{array}$ & $\begin{array}{l}5.12 \pm .02 \\
5.75 \pm .02 \\
25.1 \pm .1 \\
26.3 \pm .1 \\
32.4 \pm .1 \\
33.4 \pm .15 \\
36.4 \pm .1 \\
-11 \pm \pm . .2 \\
113.2 \pm .4 \\
158.0 \pm 1.0\end{array}$ & 5.70 & $\begin{array}{r}5.08 \\
5.76 \\
26.18 \\
27.96 \\
32.25 \\
34.0 \\
40.1\end{array}$ & $\begin{array}{l}\beta \\
\beta \\
\rho \\
\rho \\
\sigma \\
\pi \\
\rho \\
\omega \\
\nu \\
\phi\end{array}$ & $\begin{array}{l}5.12 \pm .02 \\
5.76 \pm .03 \\
25.1 \pm .1 \\
26.3 \pm .1 \\
32.35 \pm .1 \\
33.4 \pm .1 \\
36.45 \pm .1 \\
76.0 \pm .4 \\
113.4 \pm .4 \\
158.7 \pm 1.0\end{array}$ & $\begin{array}{l}5.03 \\
5.63 \\
24.2\end{array}$ & $\begin{array}{r}4.95 \\
5.65 \\
25.76 \\
29.5 \\
30.8 \\
38.5\end{array}$ & $\begin{array}{l}\beta \\
i p \\
\rho \\
\rho \\
0 \\
\pi \\
p \\
\nu \\
\nu \\
\phi\end{array}$ & $\begin{array}{l}5.10 \pm . \\
5.74 \pm: \\
25.0 \pm . \\
26.3 \pm . \\
32.4 \pm . \\
33.5 \pm . \\
36.4 \pm . \\
75.6 \pm . \\
113.2 \pm . \\
158 \pm 1 .\end{array}$ & $\begin{array}{ll}.1 & 5.04 \\
.1 & 5.75 \\
.2 & >25.8 \\
.2 & \\
.2 & \\
.2 & \\
.2 & \\
.4 \\
.4 \\
.5\end{array}$ & \\
\hline
\end{tabular}


[112] direction

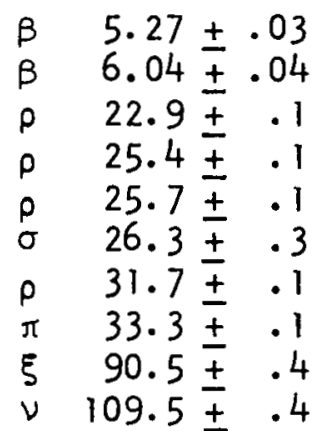

[ill] direction

$\begin{array}{lrrr}\beta & 5.41 \pm .03 & 5.37 & 5.45 \\ \rho & 24.0 \pm .1 & & 24.45 \\ \rho & 30.2 \pm .1 & & 30.9 \\ \sigma & 35.2 \pm .2 & & 36.4 \\ \pi & 35.4 \pm .2 & & \\ \xi & 84.7 \pm .4 & & \\ \nu & 103.5 \pm .4 & & 108.6\end{array}$

[121] direction

B $\quad 5.28 \pm .1 \quad 5.28$ $5.98 \div .15 .97$ $23.0 \pm .15$ $25.2+.3$ $26: 1 \mp .4$ $27.6 \mp .4$ $31.7 \pm .2$ $33.6+.3$

$\xi \quad 89.0 \mp 1.0$

$\checkmark 108.2 \pm 1.0$ 
in the $(1 \overline{1} 0)$ plane, where the data has come from both samples, is a measure of our ability (1) to place each of the two samples in the ( $1 \overline{0} 0$ plane, (2) to apply the same angle correction for a given dial reading on the sample holder, and (3) to pick a symmetry direction for the folded data at $0^{\circ}$ and $90^{\circ}$. Once these three things have been done, there are no additional variables at our disposal to enable us to do a better job of making the data from these two samples overlap.

In Figure 6 the frequency branches labelled $\beta$ arise from orbits around the 6 electron lenses situated at each of the symmetry points $\Delta$ in the Brillouin zone. The $\rho$ branches arise from the orbits associated with the 12 ellipsoids situated at the symmetry points $N$. The single frequency branch $\nu$ comes from orbits around the 6 equivalent octahedra located at the points $H$.

The rest of the orbits can be identified with the electron jack at $\Gamma$ in the center of the zone. The frequency branches $\pi$ and $\sigma$ correspond to orbits around the balls and necks respectively. The $\tau$ frequency branch arises from a central orbit around four balls of the jack. The $w$ frequency branch, due to a non-central orbit containing part of two adjacent balls and the body between them, is shown in the (110) plane in Figures 6 and 9 and should also appear in the (001) plane at [110] and exhibit some angular dependence. Although this frequency branch probably existed in the raw data, it could not be sorted out from the pattern of 2 nd and 3 rd harmonics arising from data in the 25-40 MG range, and was therefore omitted. The two data points between $71^{\circ}$ and $73^{\circ}$ which look like they could be part of the $\xi$ frequency branch actually belong to the end of the 
$\omega$ frequency branch, as determined by comparison of their amplitudes with those of nearby data points in the two branches. Then the unlabelled frequency branch which appears at angles between the $\xi$ and $\phi$ frequency branches can be explained as arising from a non-central orbit probably encompassing the body and one ball of the jack as an intermediate between the $\xi$ frequencies coming from orbits around the body of the jack, and the $\phi$ frequencies coming from an orbit encompassing two of the balls and the body between them.

Data for the (1iil) plane is shown in linear plots in Figures 10 and 11. Note the break in the frequency scale in Figure 10. The right half of this figure is missing some data because data was taken over a shorter angular range at high field for this plane. The angular scale shown with tick marks every $10^{\circ}$ was that used in determining a symmetry direction for three frequency branches chosen for use in inversion of ellipsoid and octahedron data (see sections $111-B$ and $111-C$ ). It is obvious that this data does not have the symmetry which it should, and therefore, the data has not been folded about the symmetry direction. However, this plane is extremely sensitive to small misalignments of the sample. If the sample is only $1^{\circ}$ out of the (Iiil) plane, the four frequencies which should all cross at 25.1 MG at exactly [110] will not cross there, but will be shifted. The new crossing point for those frequency branches lying entirely between 24 and $27 \mathrm{MG}$ in this plane can be greater than $10^{\circ}$ from [110]. These three frequency branches in the data exhibit the most deviation from the expected symmetry pattern. Notice also the $2^{\circ}$ uncertainty in the exact position for the [110] direction. A second attempt to 
orient the sample in this plane resulted in data almost identical to that presented here. We estimate that an alignment error of less than $1^{\circ}$ can account for the symmetry problems shown by this plane, and use this fact along with the success of our data in the other two orientation planes to determine the uncertainty of the plane of orientation of a sample with respect to the magnetic field as $1^{\circ}$ or less. 


\section{DISCUSSION OF THE RESULTS}

A. General Considerations

The experimental information about the FS of a metal obtained through the detection of the dHvA frequencies is in terms of extremal cross-sectional areas of the FS normal to the applied magnetic field (Equation i). The FS information that one would really like to see would be the specification of the radius vector to any point that lies on the surface. Such information would constitute a complete geometric description of the Fermi surface.

A quick qualitative comparison of the Fermi surfaces of $W$ and Mo can be made through the use of Figure 12. The plot exhibits the Mo data from Figure 6 as solid lines and the $W$ data of Girvan et al. (22) as dashed lines. The band structures for these metals are similar, so qualitatively similar Fermi surfaces are to be expected, except that $W$ does not have lenses. The octahedra of both metals compare rather closely in size. The electron jack of Mo is quite a bit larger in body, ball and neck dimensions than in $W$. The Ienses in Mo add a small contribution to the volume of the total electron FS pieces. The hole ellipsoịds are much larger in Mo than in $W$, which is to be expected if the hole volume is to exactly compensate for the increased electron jack volume and the volume due to the lenses. Remember that for each of these compensated metals the volumes of the electron FS pieces must equal the volumes of the hole FS pieces.

There are at least three ways of relating FS dimensions to the dHvA data. (1) An accurate band structure calculation for Mo could be done from which FS radi i can be obtained. The intersection of a plane with 


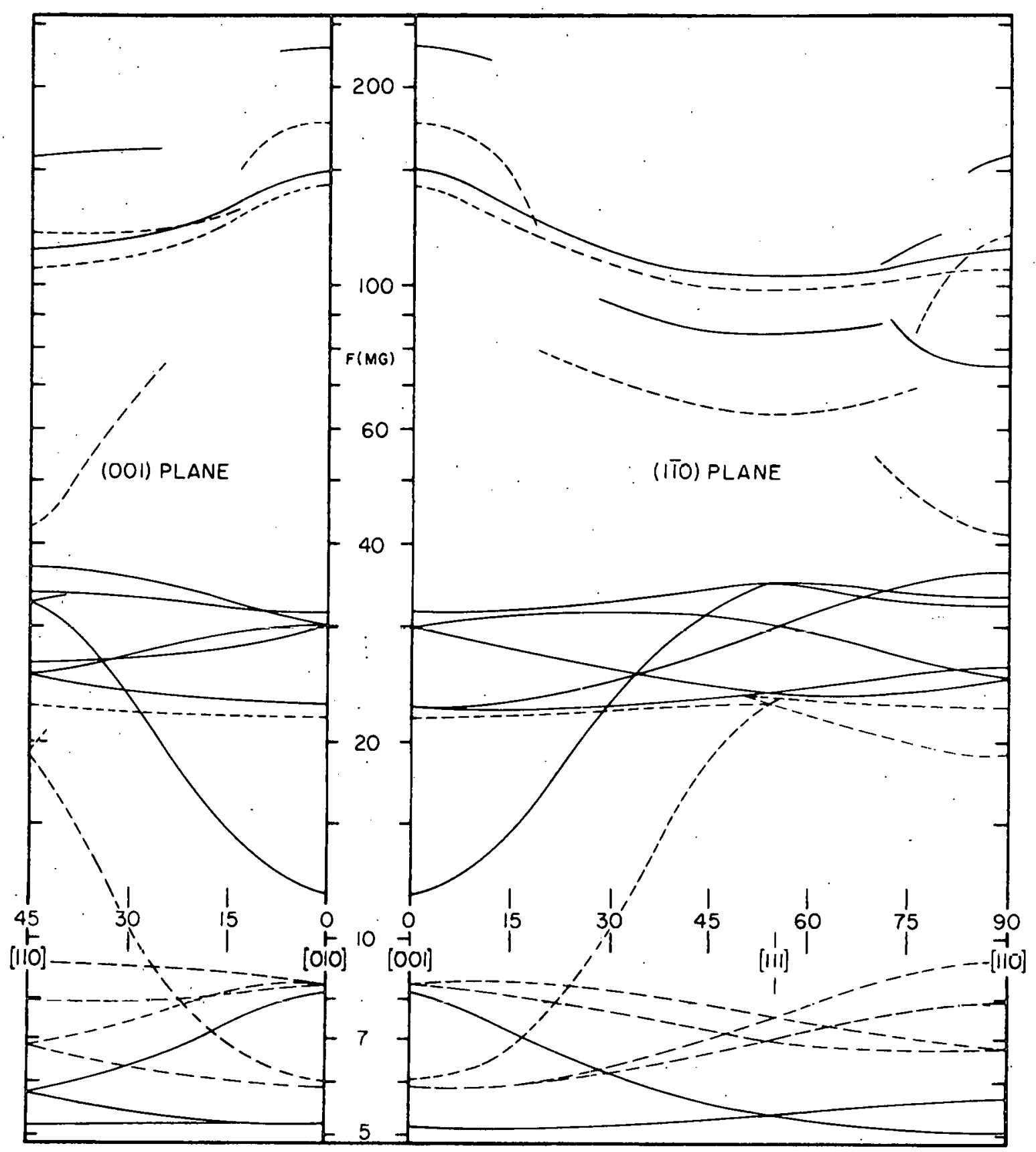

Figure 12. Comparison of the de Haas-van Alphen frequencies of molybdenum and tungsten. The solid lines indicate molybdenum data from this study. The dashed lines indicate tungsten data from Reference 22 
these radii can be integrated to determine the extremal areas one would expect to see in a dHvA experiment. The calculated areas can be compared with the experimental data and conclusions drawn about the accuracy of the band structure calculation and about how consistent the data really is. (2) The FS areas could be converted into FS radii by some inversion technique. The hole ellipsoids at $\mathrm{N}$ and the hole octahedron at $\mathrm{H}$ satisfy the requirements needed to invert the dHVA data to obtain FS radii by using computer programs (34) implementing. the Mueller inversion scheme (35). These requirements for a unique inversion are that the surface must: (a) be closed, (b) have a center of inversion symmetry, and (c) have a unique radius vector from that center. The lenses and jack fail requirements (b) and (c) respectively. (3) simple or complex geometrical models can be devised at the appropriate symmetry point and used to predict experimental extremal cross-sectional areas. Sometimes the models can be revised until the desired accuracy of $f i t$ to the data is obtained.

An accurate band structure calculation has not yet been done nor has anyone predicted the dHvA frequencies as a function of magnetic field direction, except for symmetry directions. The APW calculations of Loucks (4) show qualitative agreement with the Lomer model for Mo, but the numerical results are questionable (see Section 1), so we will not compare our results with theoretical numbers.

The inversion scheme programs (34) will be used for the octahedra and the ellipsoids, but san not be used on the lenșes and jack. The difficulties encountered in applying the inversion scheme and the criteria for picking the number of coefficients to use in the expansion will be 
discussed shortly.

The 11 parameters of the fairly complex geometrical model for the $W$ jack (see Reference 22) were adjusted in an attempt to fit the Mo data. A program used to generate this model was obtained from Dr. R. F. Girvan (36). While a few of the parameters could be successfully chosen to fit data coming from the body of the jack, no complete set was found that would even come within $5 \%$ of all the data points available at high symmetry directions. The choosing of the parameters is done by hand, since the equation is highly non-linear and the data are compared to integrals of the equation. This is not to say that a better set of 11 parameters does not exist, but the likelihood of finding this better set is quite small. Therefore, we shall attempt to use only very simple geometrical models to determine the dimensions of the jack and compare these with the results of other experiments.

A significant error exists in the Appendix B of Reference 34 containing the inversion programs. The Kubic Harmonic Expansion Coefficients, necessary for use with the $o_{h}$ group inversion programs, has the leading two columns of each page missing. These columns should contain "sign" information about several of the numbers in the table. The first coefficient in the table also has the digit "l" dropped, which is necessary to obtain any results at all from programs using this information. A partial list of these coefficients in Reference 37 was used in detecting this error and the complete list of correct values was later obtained from the program authors. One parameter, the cone angle, must be set at or near $90.0^{\circ}$ rather than at $0^{\circ}$, which one might try as a first guess, since no "usual" 
value is quoted to guide the user of the programs.

The symmetry at $\mathrm{N}$ is such that eight frequency branches for the ellipsoids are obtained from data taken in only the (001) and (170) planes. The number of expansion coefficients uniquely determined by the data supplied to the inversion program is related not only to the number of data points, but also to the angular position at which the points are taken. Choosing to take data only in planes of high symmetry does place a limit on the number of coefficients which should be used for inversion. A general feeling for this limit develops as attempts are made to determine increasingly larger number of coefficients. For the octahedron at $H$, the full cubic symmetry $O_{h}$ applied. Here data taken in only the (001) and ( 1 io) planes can not yield unique coefficients whose $l$ value is greater than 18 (11-terms)(see Reference 37). Including additional data from the (1ii) plane should increase this number of terms somewhat. The number of coefficients used in the final fit for each surface was determined by the lesser of the maximum number obtainable and the point at which addition of more coefficients would yield no further reduction in the standard deviation of the data from that predicted by the inversion scheme.

\section{B. Hole Octahedron at $\mathrm{H}$}

The data relating to the octahedron, labelled $\nu$ in Figure 6 , from all three planes was used with the inversion scheme for the $0_{h}$ symmetry group to obtain the FS radi for this piece of the total surface. The symmetry directions chosen for the (iil) plane are those which are shown in Figure 10.

A plot of the normalized frequency difference (in percent) 


$$
\Delta F=100\left(F_{\text {inversion }}-F_{\text {data }}\right) / F_{\text {data }}
$$

as a function of angle and $\ell$ value is shown in Figure 13. The frequencies from the dHVA data were interpolated to integer angles for comparison with the integer angle output of inversion frequencies. The inversion scheme fit all of the data within $0.8 \%$ and most of the structure in this curve can probably be attributed to the scatter in the input dHvA frequencies, rather than to the errors in fitting by the inversion program.

As indicated in Section $111-A, l=18$ with 11 coefficients is the highest unique fit for data from only the (001) and (1.i0) planes. Using the radii determined from an $l=18 \mathrm{fit}$ using data from all three planes as a standard, we compare the radii obtained for higher values of $\ell$. A plot of these radi i differences (in percent)

$$
\Delta R=100\left(R_{l}=20,22 \text {, or } 24^{-} R_{l}=18^{/ R} l=18\right.
$$

as a function of angle is shown in Figure 14. We notice that none of the radi $i$ have changed by more than $\pm 2 \%$ as the $l$ value has been increased. From the number of peaks and valleys in the $\ell=24$ curve, we gather that the fit is probably sensitive to large changes in radii (surfaces of high curvature) only for changes occurring over angular intervals of approximately $10^{\circ}$ or more.

The cross sections of the octahedron pieces of the FS for the (001), (1i0), (1i1) and $(1 \overline{1} 2)$ planes are shown in Figures 15 and 16 for the $\ell=24 \mathrm{fit}$ involving 19 coefficients. The 19 coefficients are 1 isted in Appendix A. The $l=24 \mathrm{fit}$ was chosen as the cut-off point where using 


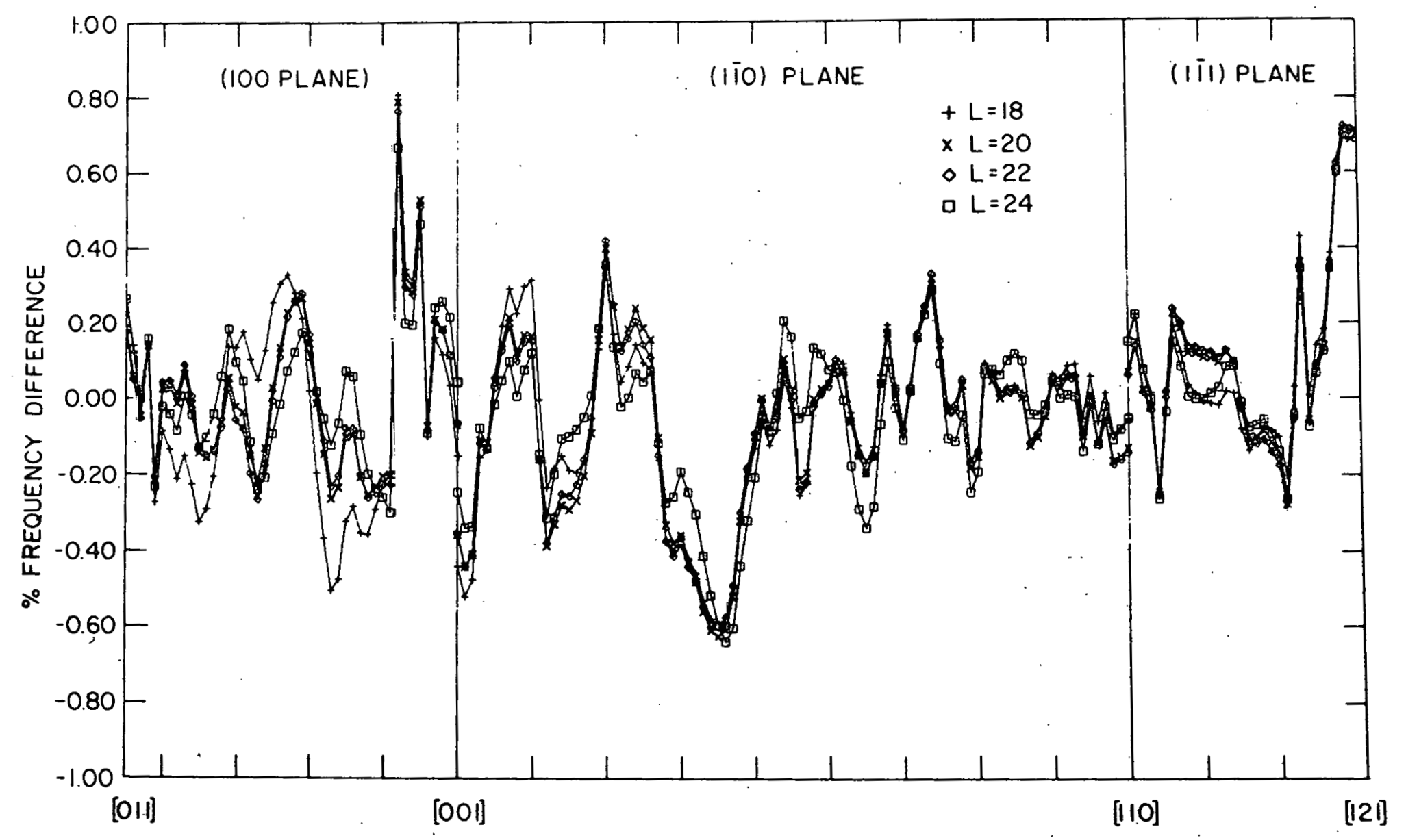

Figure 13. Plots of the normalized frequency difference (in percent) $\Delta F=100\left(F_{\text {inversion }}-F_{\text {data }}\right.$ )' $F_{\text {data }}$ as a function of angle and $\ell$ value 

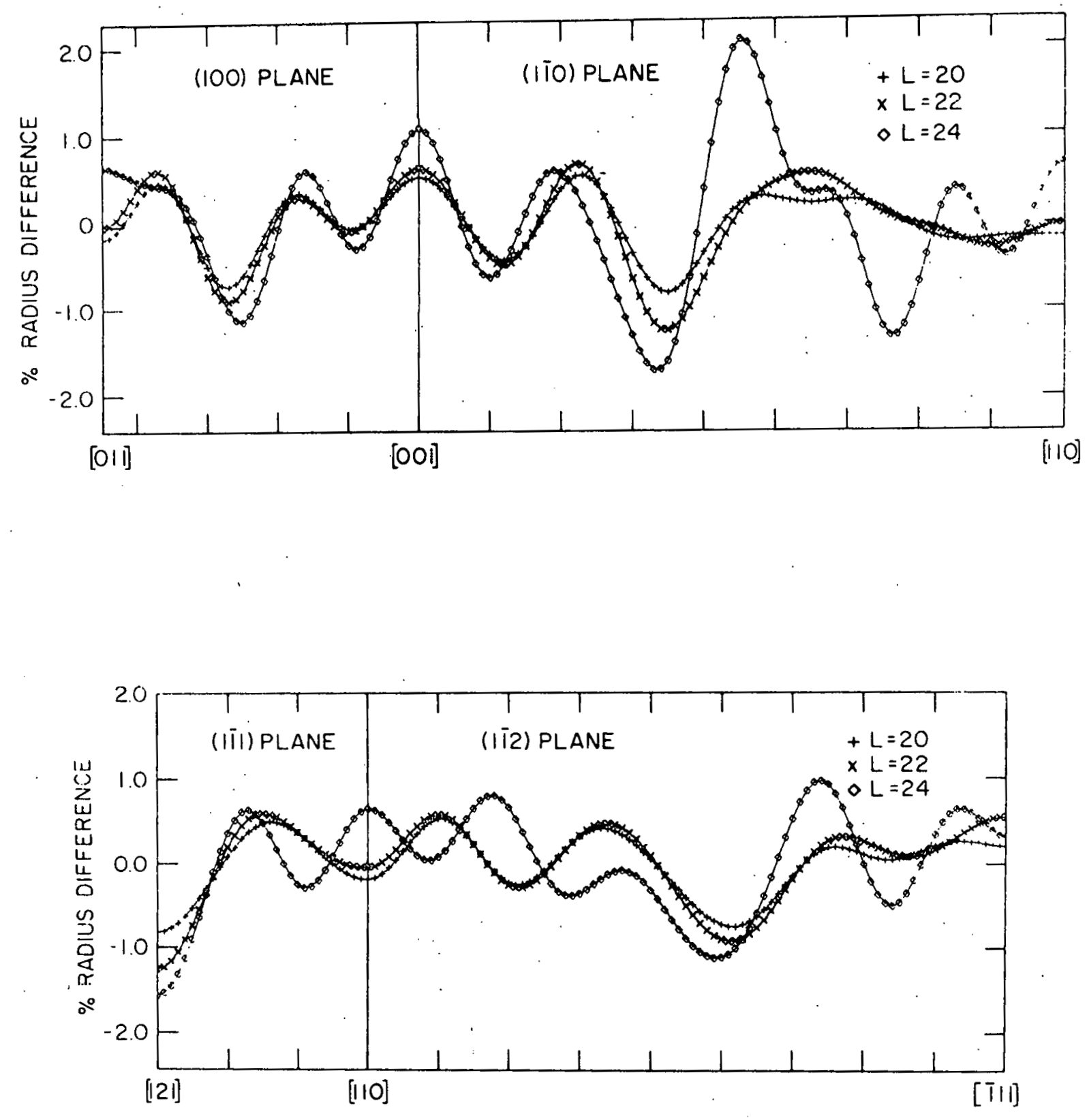

Figure 14. Plots of the radii differences (in percent) $\Delta R=100\left(R_{l}=20,22 \text { or } 24^{-} R_{l}=18\right)^{/ R_{l}}=18$ as a function of angle 

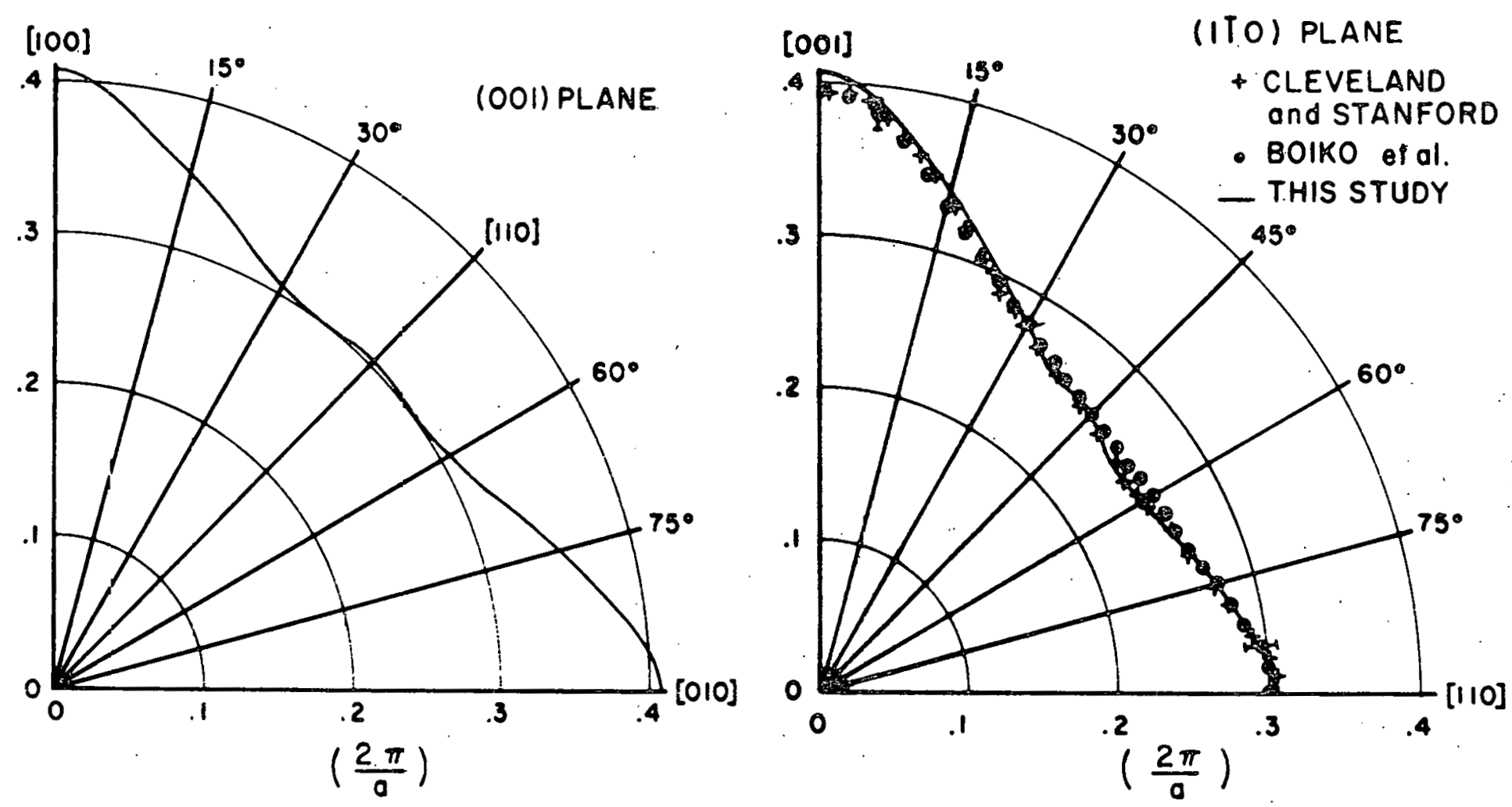

Figure 15. Cross sections of the hole octahedron Fermi surface at $H$ for the (001) and (Iī0).planes. The RFSE data of Boiko et al. (18) has been plotted directly, while the RFSE data of Cleveland and Stanford $(20)$ has been multiplied by 1.05 before plotting 

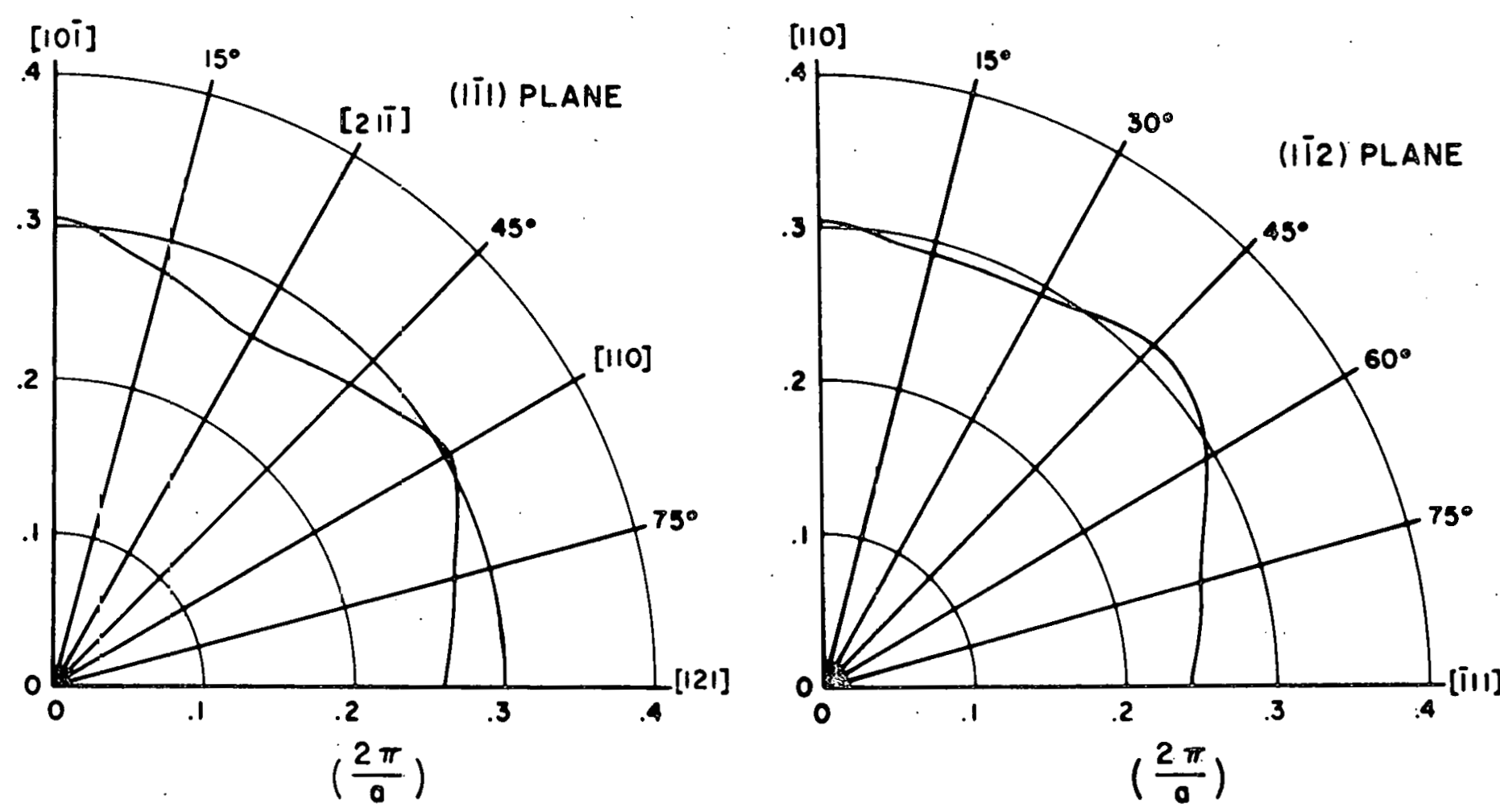

Figure 16. Cross sections of the hole octahedron Fermi surface at $H$ for the $(1 \overline{1})$ and (iiz) planes 
additional coefficients would not further reduce the standard deviation of the input data from that predicted by the inversion scheme. In the (II0) plane in Figure 15, the RFSE caliper dimensions of Boiko et al. (18) have been plotted directly, while the dimensions obtained by cleveland (19) and Cleveland and Stanford (20) have been multiplied by 1.05 before plotting. In the (iTo) plane the RFSE calipers for this piece of the FS are also the FS radii, except for angles near $50^{\circ}$, where the calipers would not be able to measure the slight dimple in the surface near these angles, and would, therefore, yield larger values than those of a plot of radii. At the [001] direction the RFSE measurement is probably more accurate than the radii indicated by the inversion scheme, since the surface has high curvature near this direction.

Radii obtained from inversion of the dHVA data for the octahedron are tabulated for major symmetry directions in Table 2. The volume

Table 2. Radi $i$ for the hole octahedra in units of $(2 \pi / a)$

\begin{tabular}{cccc}
\hline Direction & $\begin{array}{c}\text { Inversion } \\
\text { schemc } \\
\text { radii }\end{array}$ & Boiko et al. & $\begin{array}{c}\text { Others } \\
\text { \& stanford } \\
(20)\end{array}$ \\
\hline$\langle 100\rangle$ & .407 & .395 & .376 \\
$\langle 110\rangle$ & .304 & .30 & .290 \\
$\langle 111\rangle$ & .244 & .255 & .238 \\
$\langle 112\rangle$ & .262 & .27 & .253 \\
\hline
\end{tabular}


calculated using the inversion coefficients in a suitable program from Reference 34 was found to be $0.90 \AA^{-3}$. This compares with $0.90 \AA^{-3}$ obtained by Boiko et al. (18) and $0.95 \AA^{-3}$ estimated by Sparlin and Marcus (16). From this comparison with RFSE data we would agree. with the measurements of octahedron radii by Boiko et al. to within errors of the experiments. Our plots of radii are probably accurate to $\pm 1 \%$, except near the [001] direction, where the errors are probably closer to $\pm 2 \%$.

The RFSE octahedron dimensions reported by Cleveland and Stanford (20) for the (110) plane were integrated by computer and yielded an area of $0.98 \pm 0.02 \AA^{-2}$. When these dimensions were increased by 1.05 , the resulting area matched the $1.083 \pm .01 \AA^{-2}$ cross-sectional area for this direction obtained in the present experiment. From the manner in which these RFSE dimensions, plotted in Figure 15 after being multiplied by 1.05, compare with this study and with the RFSE calipers of Boiko et.al., it would appear that the work of Cleveland and Stanford was subject to a systematic error of 5\%. In Reference 20 they are aware that their results do not agree with those of other investigators and have considered in detail the sources of error in their experiment. Their investigation was thorough and does not contain obvious weaknesses. Using samples cut from the same single-crystal rod, we present experimental data which disagrees with their work, but must conclude as they did concerning their work that "the reasons for the disagreement are at present unknown." (20).

\section{Hole Ellipsoids at $\mathrm{N}$}

All of the data labelled $p$ in Figure 6 comes from the hole ellipsoids at $N$. These frequency branches, four from the $(001) \mathrm{plane}$ and four from 
the $(1 \overline{1} 0)$ plane, were used as input to the inversion scheme for the $V_{h}\left(D_{2 h}\right)$ symmetry group along with the two frequency branches from the (III) plane which are the most insensitive to small errors in sample orientation. These latter two were the branches connecting the 31.7 MG frequency at the $\langle 112\rangle$ directions with either the $36.4 \mathrm{MG}$ or $25.0 \mathrm{MG}$ frequencies at the [110] direction as shown in Figure 10 with the choice of symmetry directions as indicated on that figure.

There are 12 symmetry points $\mathrm{N}$ in the Brillouin zone in Figure 1 , and generally six frequency contributions to the dHvA effect for an arbitrary magnetic field direction from a FS piece appropriately situated at equivalent pairs of these points, but we must concentrate on a single point $\mathrm{N}$ in order to obtain results from the inversion scheme. The problem can be viewed as one in which we place an ellipse with axes a, b, and c along three coordinate directions $k_{x}, k_{y}$, and $k_{z}$ with point $N$ at $(0,0,0)$ and act as if the four frequency branches from the (001) plane had actually come from four separate planes of observation on the single ellipse, and similarly for any other experimental plane of observation. As a check on the data assignments made, computed dHvA data generated from a perfect ellipsoid were fed into the inversion scheme and checked with the areas calculated by the inversion scheme. The appropriate choice of spherical mapping coefficients could also be determined with this ideal data.

The three principal cross sections for the ellipsoid pieces situated at symmetry points $\mathrm{N}$ are shown in Figure 17 with different plotting symbols for each of the cross sections. The solid curves are plots of perfect ellipses having major and minor axes identical to those obtained from the 


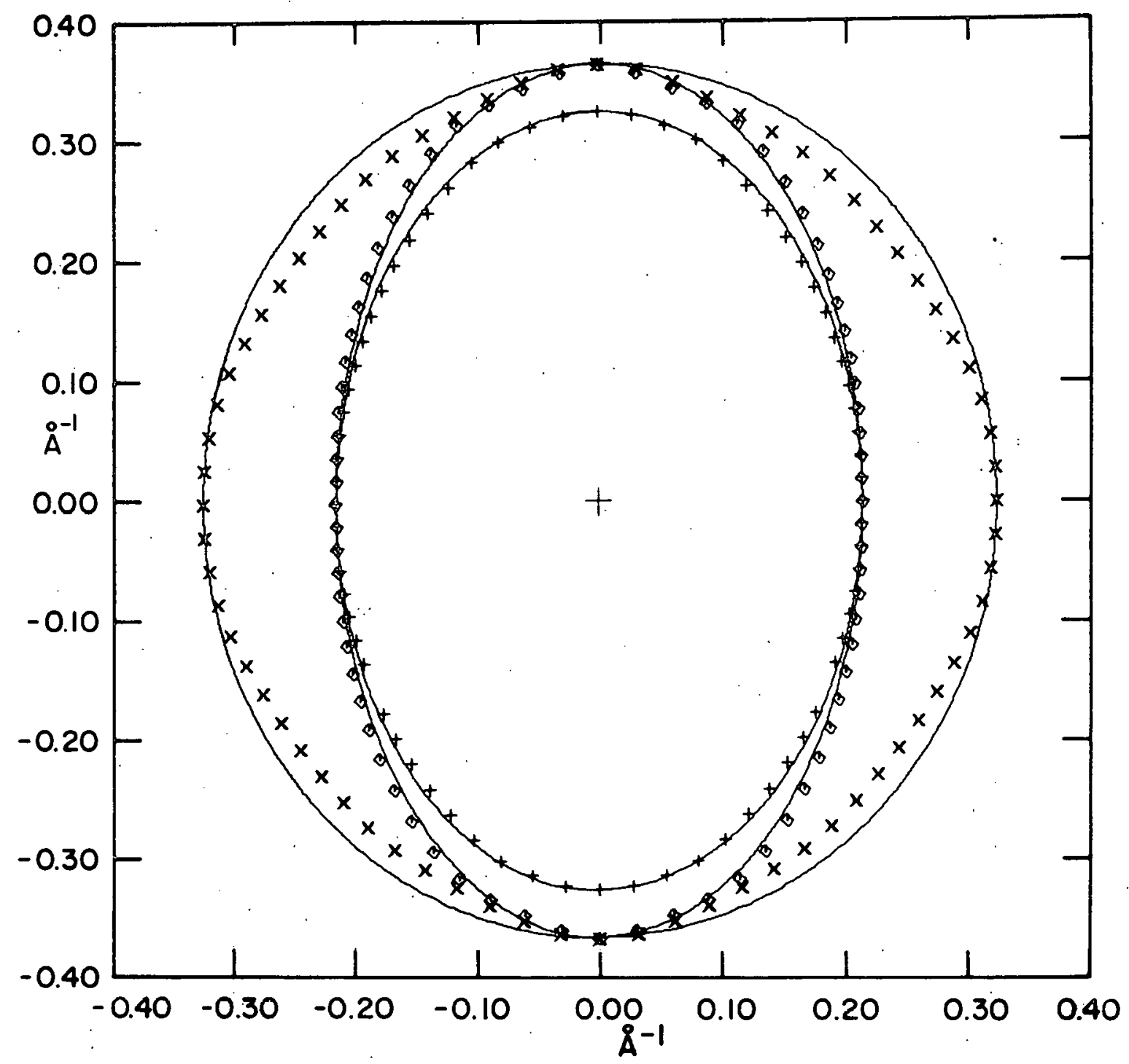

Figure 17. Cross sections of the hole ellipsoid Fermi surface at $N$ for the planes containing the three semiaxes. These directions are $\mathrm{N} \Gamma, \mathrm{NH}$, and $\mathrm{NP}$ 
inversion scheme for the $l=16$ fit involving 45 coefficients. These 45 coefficients along with the two spherical mapping parameters, ALPHA and GAMMA, are listed in Appendix A. The $l=16 \mathrm{fit}$ was the highest-order fit that could be obtained and was chosen because the standard deviation of the input data from that predicted by the inversion scheme was lower than that of any other fit obtained for a lower $\ell$ value.

Values for the three semi-axes of the ellipsoids at $N$ as found with the inversion scheme are listed in Table 3. Since it is impossible to decide upon the assignment of semi-axes to the $\mathrm{NH}$ and $\mathrm{N} \Gamma$ directions from the IHvA data, we assume that the shortest semi-axis is along NH, which is consistent with the FS calculations for Mo by Loucks (4). The volume calculated using the inversion coefficients in a suitable program from Reference 34 was found to be $0.614 \AA^{-3}$ for 6 hole ellipsoids. This compares with $0.61 \AA^{-3}$ obtained by Boiko et al. (18) and $0.71 \AA^{-3}$ obtained by Sparlin and Marcus (16).

Table 3. Values in $\AA^{-1}$ for the semi-axes of the hole ellipsoids at $N$

\begin{tabular}{|c|c|c|c|c|c|}
\hline \multirow[t]{2}{*}{ Direction } & \multirow{2}{*}{$\begin{array}{c}\text { Inversion } \\
\text { scheme } \\
\text { radii }\end{array}$} & \multirow{2}{*}{$\begin{array}{l}\text { Estimated } \\
\text { from } \\
\text { three } \\
\text { areas }\end{array}$} & \multicolumn{3}{|c|}{ Other investigations } \\
\hline & & & $\begin{array}{c}\text { Boiko et al. } \\
(18)\end{array}$ & $\begin{array}{c}\text { Cleve land } \\
\text { E Stanford } \\
(20)\end{array}$ & $\begin{array}{l}\text { Sparlin } \\
\varepsilon \text { Marcus } \\
(16)\end{array}$ \\
\hline NP & 0.365 & 0.358 & 0.38 & 0.35 & $0.39 \pm .01$ \\
\hline$N \Gamma$ & 0.325 & 0.310 & 0.29 & 0.32 & $0.30 \pm .01$ \\
\hline $\mathrm{NH}$ & 0.216 & 0.223 & 0.22 & 0.20 & $0.23 \pm .01$ \\
\hline
\end{tabular}


The column labelled "estimated from three areas" in Table 3 contains semi-axes as calculated from the frequencies (and corresponding areas) which exhibit the condition that $\mathrm{dF} / \mathrm{d} \theta=0$ at $\langle 110\rangle$ or $\langle 100\rangle$ directions. These three frequencies correspond to the extremal cross sections rab,

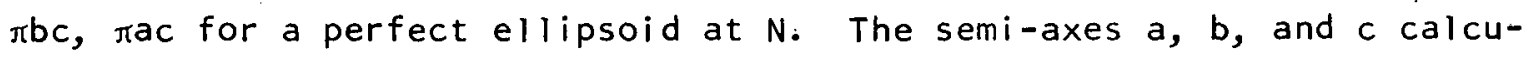
lated in this manner can only be as good as the approximation that the actual surface is a perfect ellipse. As we can see from figure 17, the largest cross section has some places which are flatter than the perfect ellipse with same semi-axes, so we would expect semi-axes estimated in this manner to differ from those determined by the inversion scheme. The inversion scheme radi $i$ for this surface should be accurate to about $1 \%$.

\section{Electron Lenses at $\Delta$}

Data related to the electron lenses at symmetry point $\Delta$ along $\Gamma H$ in the Brillouin zone cannot be inverted by Mueller's inversion scheme, since this point does not possess inversion symmetry. The physical significance of this fact is that dHvA experiments cannot provide enough information about this surface to specify a unique surface consistent with the required $\mathrm{C}_{4 \mathrm{v}}$ symmetry. If an additional condition is imposed that the surface has a mirror.plane whose normal is parallel to $\Gamma H$, the point has: now been given the $D_{4 h}$ symmetry, which satisfies inversion theorem requirements, and enables use of the Mueller inversion scheme or use of a geometrical model with the additional symmetry. However, we may only obtain a possible substitute for the actual surface when we impose this additional requirement to obtain a unique fit to the data. It is possible, but rather unlikely, that the real FS exhibits this mirror symmetry. 
The condition of four-fold symmetry in a plane whose normal is parallel to $\Gamma H$ for the point $\Delta$ and the almost zero slope of the lowest frequency branch of the experimental data for the (001) plane (see Figure 7) can be combined to suggest that the lenses are very nearly circular with radius $r$ in a cross section whose normal is parallel to $\Gamma$. The dHvA data about the lens' cross section having a dimension d along $\Gamma H$ as one axis and the lens' radius $r$ as the other axis is not sufficient to specify the distance $\alpha$ between the center of the plane of the circular cross section and the center of the $\Gamma H$ dimension. It can be shown by simple geometrical arguments that two different cross sections with identical values for $r$ and cross-sectional areas, but differing in values of $\alpha$ and $d$ should be distinguishable because of differing caliper dimensions for the same angle of observation.

This effect is probably only noticeable as a $4-5 \%$ difference in caliper dimensions, unless the lenses are highly asymmetrical. In other words, the RFSE might be able to distinguish between symmetrical and asymetrical models which could both agree with the dHvA results. However, unless the RFSE data is very accurate, probably better than $1 \%$, the amount of asymmetry along the $\Gamma H$ direction could not be determined quantitatively. Such accuracy can not be expected from RFSE orbits yielding small caliper dimensions, since these resonances are detected at low magnetic field strengths, where $\omega_{c} \tau$ is low and the RFSE Iine shapes are broad. Another result of the geometrical arguments is that while the distance $\alpha$ can vary quite widely, the $\Gamma H$ dimension for the lens remains reasonably constant, as does the volume of the FS. 
For the above reasons, we will use the five frequency branches for the $(001)$ and $(1 \overline{1} 0)$ planes shown in Figure 7 as input to the inversion scheme for the $D_{4 h}$ symmetry group. As with the ellipsoids discussed in Section $1 / 1-C$, we pick one lens and place it at the origin of our inversion $k$-space and use all of the data as if it had been taken from five different planes of observation. The program was checked with perfect ellipsoids of revolution and the spherical mapping parameter was also determined from this ideal data.

The two principal cross sections for the lens pieces situated at symmetry points $\Delta$ are shown in Figure 18 , subject to the additional symmetry requirement necessary for inversion of the data. The results obtained from the inversion scheme for the $l=16$ fit involving 25 coefficients, which are listed along with the spherical mapping parameter GAMMA in Appendix $A$, came from the highest order fit that could be obtained. Values for the radi $i$ as found with this fit are listed in Table 4.

Table 4. Values in $\AA^{-1}$ for the radii of the electron lenses at $\Delta$

\begin{tabular}{lccc}
\hline Direction & $\begin{array}{c}\text { Inversion } \\
\text { scheme } \\
\text { radii }\end{array}$ & Boiko et al. & O.ther investigations \\
$(18)$ & Brandt E Rayne \\
\hline
\end{tabular}

For $\sim$ circular

cross section:

$\begin{array}{lccc}\text { parallel to }\langle 100\rangle & .162 & .155 & .16 \\ \text { parallel to }\langle 110\rangle & .155 & & \\ --222 & .22 & .22 \\ \text { Total TH dimension } & .222 & \end{array}$




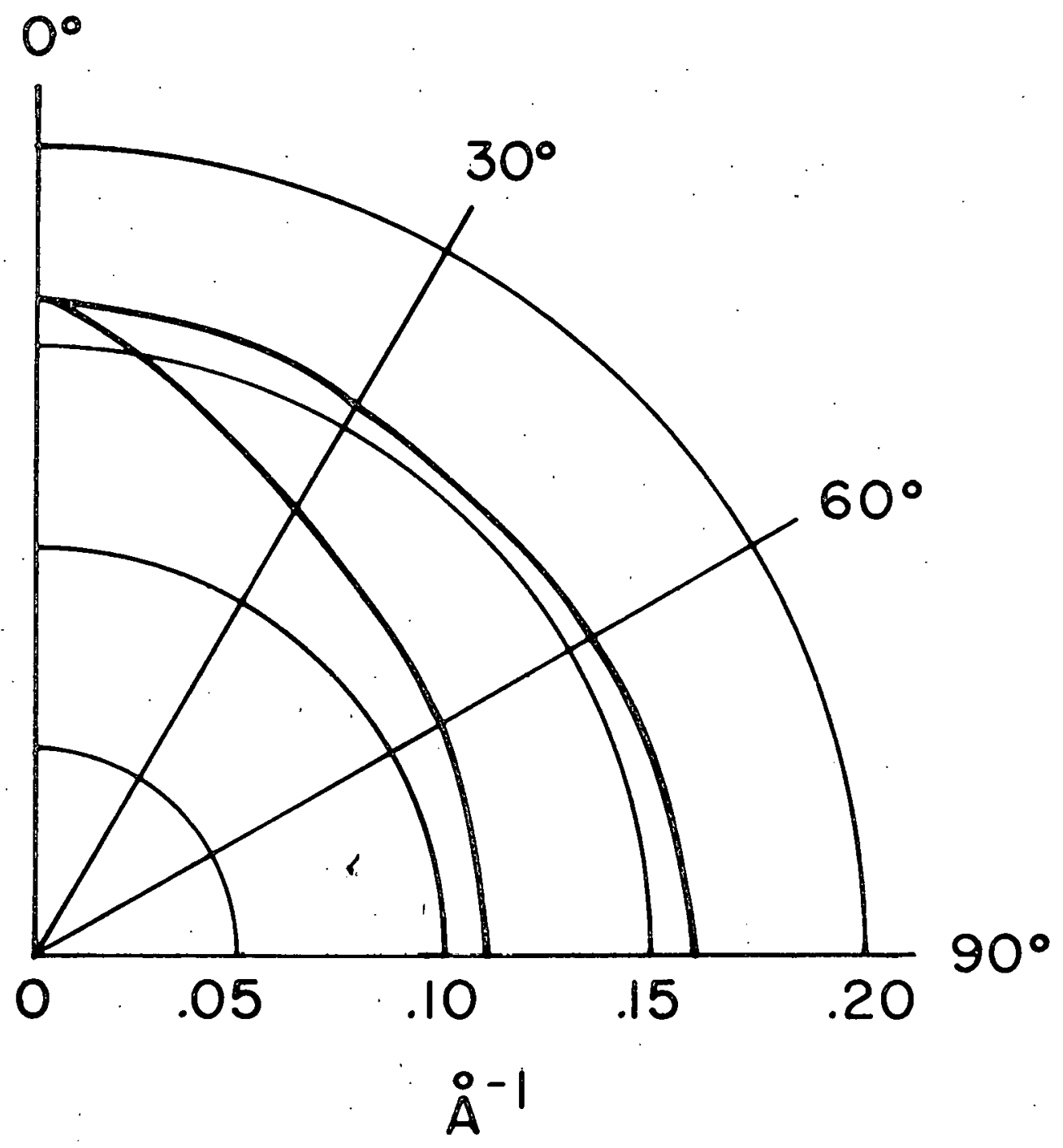

Figure 18. Cross sections of the electron lens Fermi surface at $\Delta$ assuming a higher $\mathrm{D}_{4 \mathrm{~h}}$ symmetry. For the nearly circular cross section, the plane has normal direction $\Gamma \mathrm{H}$, and $0^{\circ}$ and $90^{\circ}$ are parallel to $\langle 100\rangle$ directions 
The volume calculated using the inversion coefficients in a suitable program from Reference 34 was found to be $0.057 \AA^{-3}$ for 6 lenses. This compares with a volume of $0.060 \AA^{-3}$ obtained by Leaver and Myers (17).

\section{E. Electron Jack at $\Gamma$}

An accurate model for the electron jack surface is the most difficult to extract from the dHvA data of extremal cross-sectional areas. The data can not be inverted by using the Mueller inversion scheme because some radius vectors to points on the surface are multi-valued. There is no way to accurately determine the position of the center of the ball connected to the body portion of the jack. In Section $111-B$ we have seen that the RFSE data of Boiko et al. (18) agree quite closely with the radii obtained from inversion of the octahedron dHVA data. Their RFSE data will be used to supply some very valuable jack radii, but only at those angles where there is good reason to believe that the caliper dimensions obtained in the RFSE experiment are actually radii. The claim made by Boiko et al. (18) that "upon rotation of the magnetic field relative to the crystallographic directions in the (110) plane, the lines of the experimental points from the extremal orbits of the electron 'jack' will give directly the section of this surface with the (110) plane" is not true for the entire jack cross section in this plane. In particular the line $i$ of the data of Boiko et al. (18), shown as plotted points in Figure 19, can be coincident with the actual radius vector only at the [001] direction. At any other angular position for this line, the RFSE measures caliper dimensions, and must be interpreted as such. It is easy to fall into the trap of equating caliper dimensions with radi $i$ for 


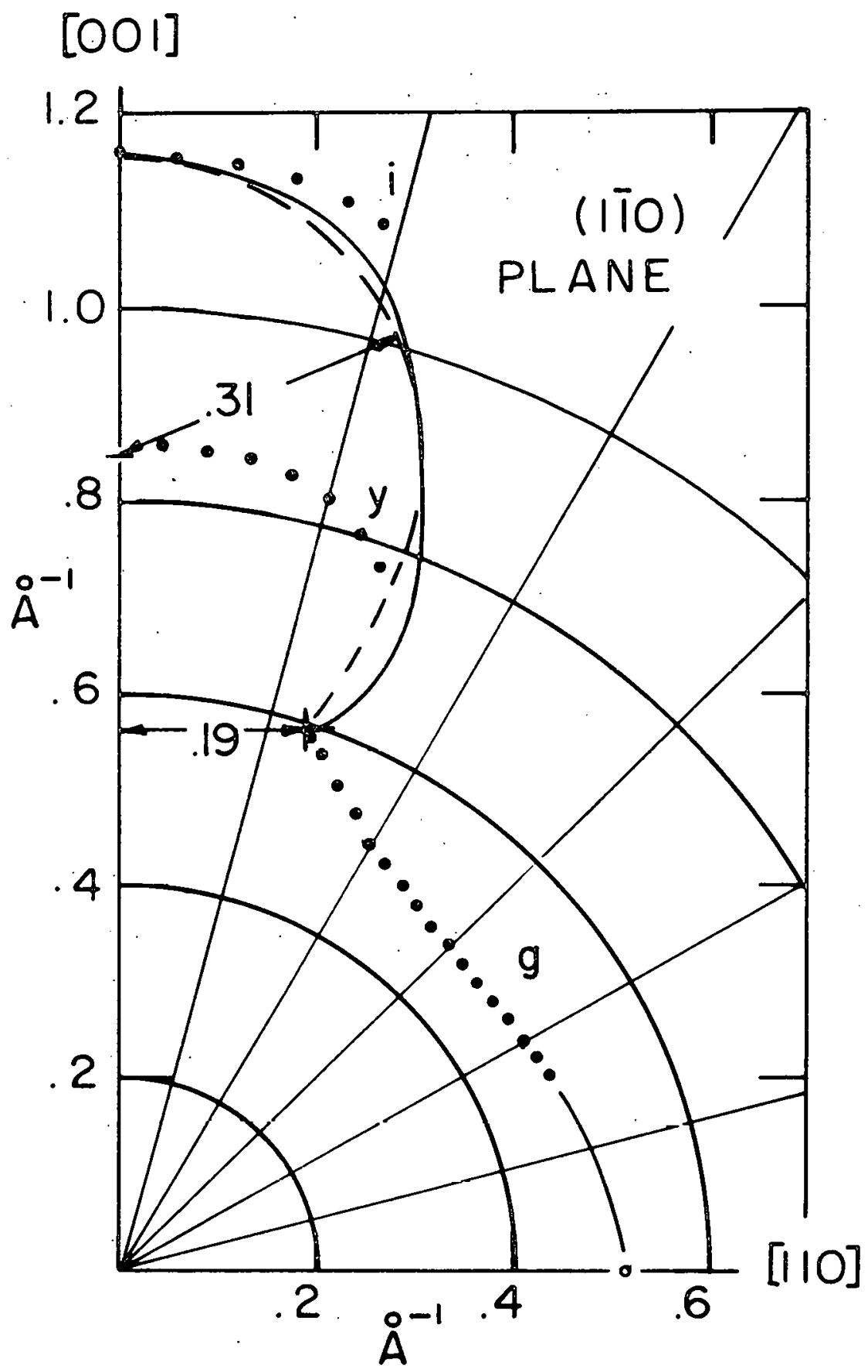

Figure 19. (1 10$)$ plane cross section for the electron jack Fermi surface at $\Gamma$. The solid and open circle data points and their labels are RFSE results of Boiko et al. (18). The + data point at $19^{\circ}$ from [001] and the solid Tine construction are described in the text 
surfaces whose curvature and symmetry does not allow. such a statement to be true. In general extreme care should be exercised when choosing the RFSE calipers which also have the property of being FS radii.

In the following paragraphs the rational for the particular (1i0) and (001) cross sections for the electron jack as shown in Figures 19 and 20 will be presented. First some facts obtained directly from our data should be noted. In Section III-D we saw that the electron lenses are very nearly circular. Since the lenses lie in the neck portion of the jack and the lens and neck cross sections are comparable in size, it is reasonable to assume that the necks are also very nearly circular. From the value of $11.7 \mathrm{MG}$ at the $\langle 100\rangle$ directions for the neck orbit labelled $\sigma$ in Figure 6 , we obtain a radius of $0.19 \AA^{-1}$ for a circular neck cross section. Looking at the (1i0) plane for the ball orbit labelled $\pi$ in Figure 6, we see values of $31.63 \mathrm{MG}$ at [001], 33.4 MG at [110], and 35.4 MG at [III]. We also note, that the neck cross section is $32.4 \mathrm{MG}$ at [110]. Assuming a rather spherical shape for the ball, and a circular cross section for the [001] direction, we calculate a radius of $.310 \AA^{-1}$ for the ball. The angles at. which the body orbit labelled $\xi$ in Figure 6 disappear in the (lio) plane can help to define the neck dimensions. At a magnetic field direction of $19 \pm \frac{1}{2}^{0}$ from [110] the neck interferes with the completion of the body orbit, and the orbit is no longer possible. This corresponds to the loss of RFSE signals at $21 \pm 2^{\circ}$ from a $\langle 100\rangle$ direction reported by $C$ leveland and $S$ tanford $(20)$, and at $21 \pm 3^{\circ}$ from a $\langle 100\rangle$ direction reported by Boiko et al. (18). Note that RFSE data are plotted as caliper dimensions for given directions and are obtained with 


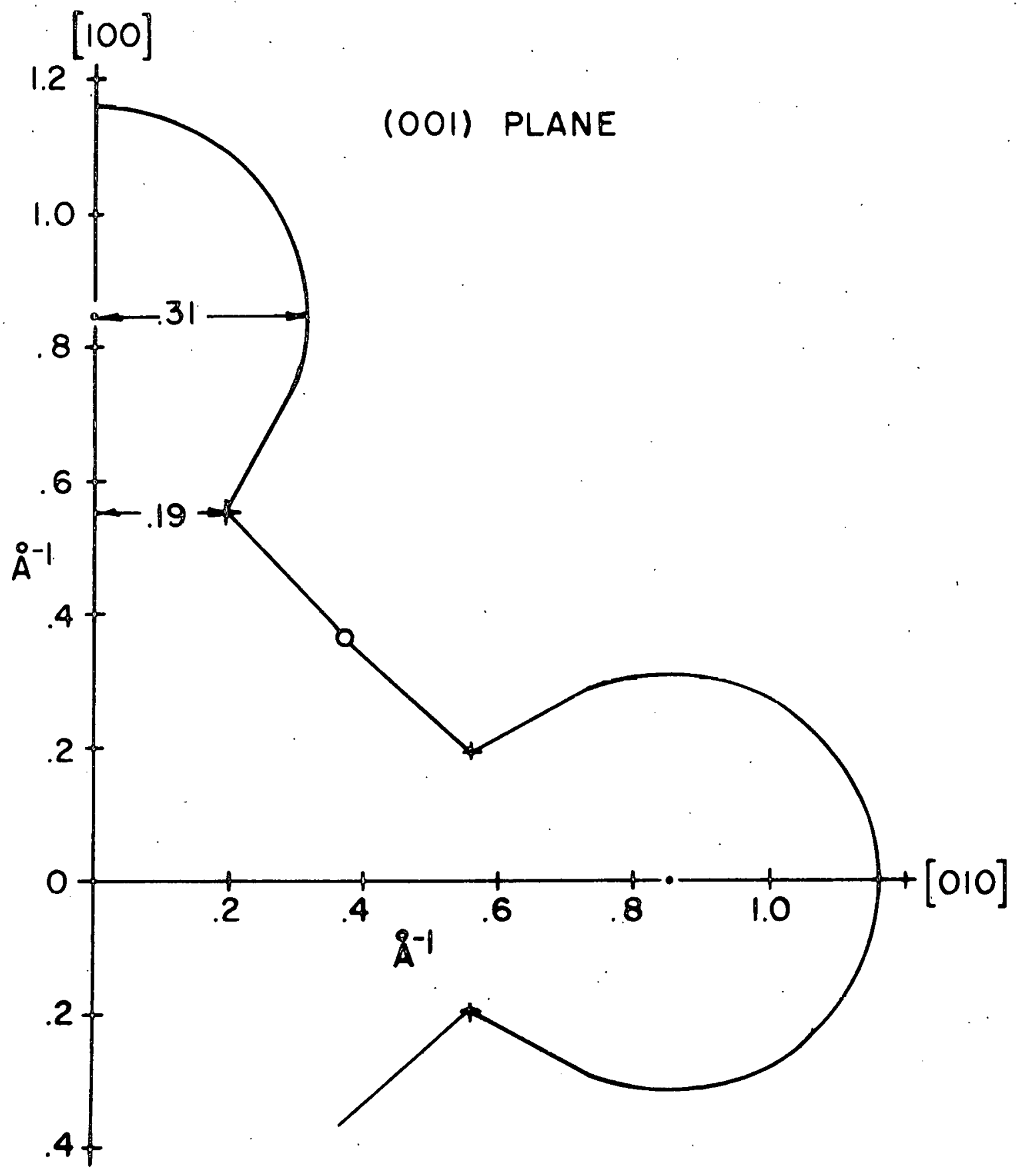

Figure 20. (00I) plane cross section for the electron jack Fermi surface at $\Gamma$. The open circle is a data point from Reference 18 . The + data points and the solid line construction are described in the text 
fields applied perpendicular to the given direction and to the plane of the sample: At a magnetic field direction of $27 \pm 2^{\circ}$ from [100], the orbit around the body changes into an orbit which encompasses all four balls. A loss of RFSE signals at $29 \pm 2^{\circ}$ from a $\langle 110\rangle$ direction was reported by $\mathrm{Clevel}$ and and Stanford, and at $24 \pm 3^{\circ}$ from a $\langle 110\rangle$ direction by Boiko et al.

In Figure 19 for the $(1 \overline{1} 0) \cdot$ plane we assume the RFSE calipers of Boiko et al. at [00I] and [110] are radii, as well as those arising from the body of the jack, labelled $\mathrm{g}$. The radi $\mathrm{i}$ are $1.16 \AA^{-1}$ at $[001]$ and $.52 \AA^{-1}$ at [110]. We extrapolate an additional data point + for the body at $19^{\circ}$ from [001] which is indicated by our data. This point also defines the neck radius at $0.19 \AA^{-1}$ for a circular neck cross section. A completely spherical ball is shown dashed in along with a straight line to connect the neck to the ball, and a gently curved solid line is drawn connecting the body data with the data point at [110]. We shall return to this figure shortly.

We continue to assume a spherical ball with circular neck and ball cross sections at $\langle 100\rangle$ directions for the ball and neck drawn in Figure 20 for the $(001)$ plane. The RFSE data point at [110] is included and a slightly curved line is drawn to connect the necks with this point. The body is slightly concave at [110] in this plane, which agrees with the observation of two neck frequencies near 32.4 MG at small angles away from $[110]$ in the $(001)$ plane, as shown in Figures 6 and 8 . The area computed for the model (001) plane is $2.19 \AA^{-2}$ which compares very favorably with $2.21 \AA^{-2}$ for the dHvA experimental area. 
An area integration of the $(i \bar{i} 0)$ plane as we have constructed it thus far yields $1.45 \AA^{-2}$. to be compared with the dHvA experimental value of $1.51 \AA^{-2}$. We can improve the value for this area and also correct the model so that both a minimum (neck) and maximum (ball) cross section is observable at the [110] direction where the experimental data shows values of 32.4 MG and 33.4 MG by drawing the additional (solid) lines for the ball. The new area for the (110) plane is $1.49 \AA^{-2}$. An integration to check the neck and ball cross sections at [110] quantitatively is impossible to do accurately, without a complete analytical description of the surface to be analyzed.

A possible cross section for the (Iii) plane is shown in Figure 21 . The radi $i$ at the $\langle 110\rangle$ and $\langle 112\rangle$ directions are the same as indicated for similar directions in Figures 19 and 20 . The model area of $0.77 \AA^{-2}$ can be compared with the experimental value of $0.81 \AA^{-2}$ for the [111] direction.

It is not possible to carry this simple geometrical model further or make additional comparisons with the experimental data since the integrations of cross-sectional areas which do not contain the center of symmetry for the figure and/or whose normal is not a direction of high symmetry are possible only with an analytical model suitable for a computer. The assumption about fairly spherical balls is also likely to break down somewhat as one adjusts the model for a more accurate fit. As mentioned in Section III-A, the analytical approach was tried with no better results than those presented above. 


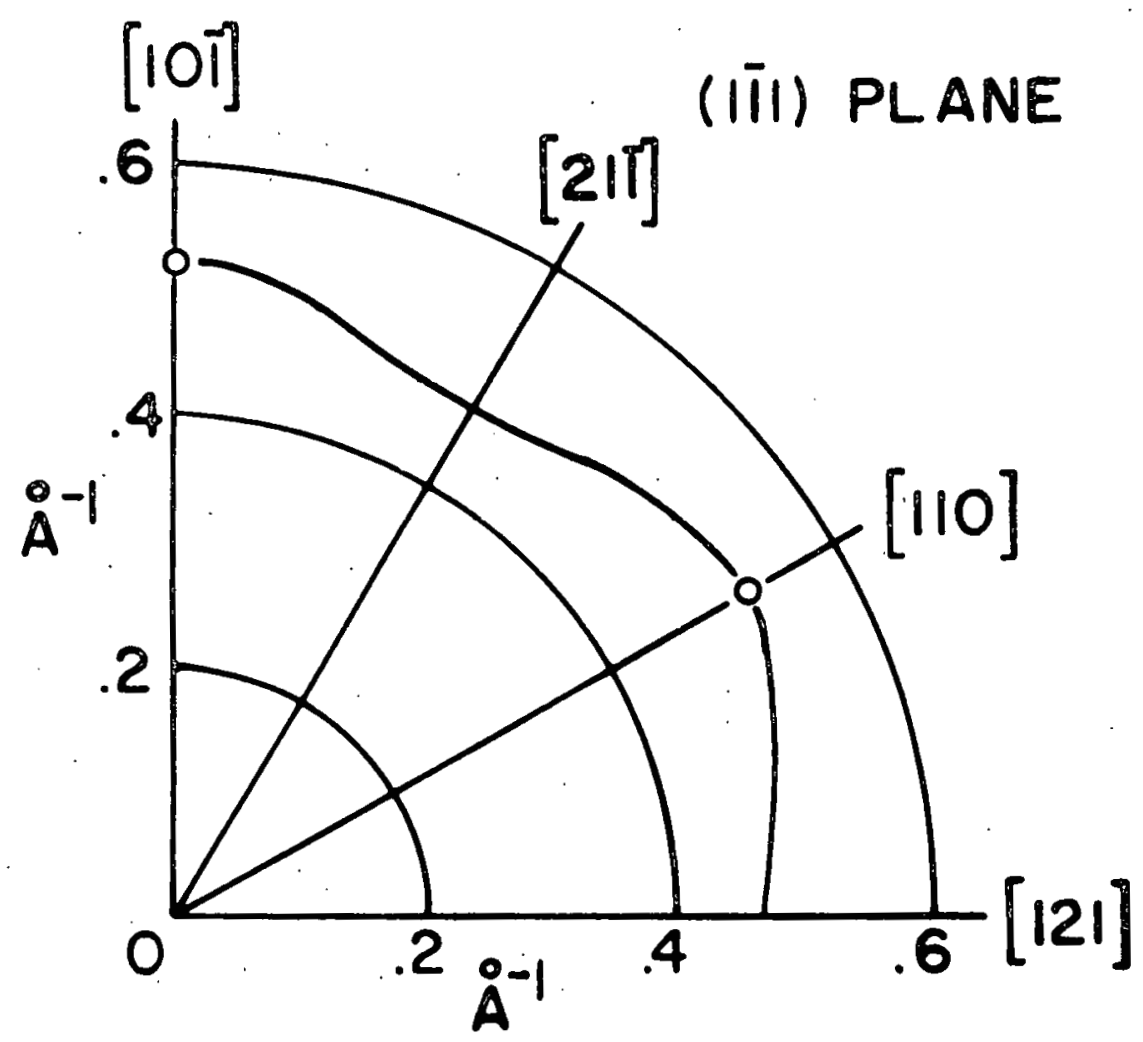

Figure 21. A possible (1il) plane cross section for the electron jack Fermi surface at $\Gamma$. The open circles are data points from Reference 18 


\section{CONCLUSIONS}

We have presented dHvA data for molybdenum which are accurate to within $1 \%$ in frequency or $1^{\circ}$ in angle, whichever is the larger error, for the magnetic field rotated in the (1i0), (001) and (1iil) crystallographic planes. Two samples, cut from the same single crystal rod as the sample used in the RFSE work of Cleveland and Stanford (20), were used to obtain the dHvA data. These data were shown to be consistent in frequency and angular determination at the appropriate major symmetry. directions for the above-mentioned planes. The data were inverted through the use of the Mueller inversion theorem (35), when possible, to obtain FS radii, and a simple geometrical model was developed for the electron jack. The FS radii for the (100) plane cross section for the hole octahedron obtained from the dHvA data were shown to be consistent with the RFSE caliper dimensions obtained by Boiko et al. (18) for the same plane. The geometrical model for the electron jack cross section is consistent with the RFSE caliper dimension at [001]. Since the inversion radii data for the octahedron are most subject to error at [001]; we believe that the determination by Boiko et al. of the RFSE caliper dimensions, which are radii at $[001]$, to be correct. These RFSE dimensions for the $\langle 100\rangle$ directions are $1.16 \AA^{-1}$ for the jack and $0.79 \AA^{-1}$ for the octahedron with the $\Gamma H$ dimension as $1.999 \AA^{-1}$, so the gap between the jack and octahedral FS pieces is estimated as $2.5 \%$ of the $\Gamma H$ dimension, thus resolving the discrepancies existing in current RFSE data.

The model presented for the electron jack could probably be improved 
upon by further calculations on a complicated analytical model. However, an accurate FS model based on band structure calculations for molybdenum would be more desirable. Such a calculation has yet to be completed by band theorists. 


\section{BIBLIOGRAPHY}

1. W. M. Lomer, Proc. Phys. Soc. (London) 80, 489 (1962).

2. J. H. Wood, Phys. Rev. 126, 517 (1962).

3. W. M. Lomer, Proc. Phys. Soc. (London) 84, 327 (1964).

4. T. L. Loucks, Phys. Rev. 139, Al181 (1965).

5. L. Hodges, lowa State University, private communication, 1971.

6. H. Jones, The Theory of Brillouin Zones and Electronic States in Crystals (North-Hol land Publishing Company, Amsterdam, 1960), p. 117.

7. E. Fawcett, Phys. Rev. 128, 154 (1962).

8. E. Fawcett and W. A. Reed, Phys. Rev. 134, A723 (1964).

9. N. E. Alekseevskiì, V. S. Egorov, G. E. Karstens and B. N. Kazak, Zh. Eksperim. i Teor. Fiz. 43, 731 (1962) [English trans1.: Soviet Phys.-JETP 16, 519 (1963)].

10. E. Fawcett and D. Griffiths, J. Phys. Chem. Solids 23, 1631 (1962).

11. C. K. Jones and J. A. Rayne, in Low Temperature Physics LT9, edited by J. G. Daunt, D. V. Edwards, and M. Yaqub (PIenum Press, Inc., New York, 1965), p. 790.

12. P. A. Bezuglyi, S. E. Zhevago, and V. I. Denisenko, Zh. Eksperim. i Teor. Fiz. 49, 1457 (1965) [English transl.: Soviet Phys.-JETP 22, $1002(1966)]$.

13. R. Herrmann, Phys. Stat. Sol. 25, 661 (1968).

14. G. B. Brandt and J. A. Rayne, Phys. Letters 3, 148 (1962).

15. G. B. Brandt and J. A. Rayne, Phys. Rev. 132, 1945 (1963).

16. D. M. Spariin and J. A. Marcus, Phys. Rev. 144, 484 (1965).

17. G. Leaver and A. Myers, Phil. Mag. 19, 465 (1969).

18. V. V. Boiko, V. A. Gasparov, and 1. G. Gverdtsiteli, Zh. Eksperim. i Teor. Fiz. 56, 489 (1969) [English trans.: Soviet, Phys.-JETP $\underline{29}$, 267 (1969)].

19. J. R. Cleveland, Ph.D. Thesis, lowa State University, 1970 (unpublished). 
20. J. R. Cleveland and J. L. Stanford, Phys. Rev. B4, 311 (1971).

21. L. F. Mattheiss, Phys. Rev. 139, A1893 (1965).

22. R. F. Girvan, A. V. Gold, and R. A. Phillips, J. Phys. Chem. Solids 29, 1485 (1968).

23. L. Onsager, Phil. Mag. 43, 1006 (1952).

24. I. A. Lifshitz and A. M. Kosevich, Zh. Eksperim. i Teor. Fiz. 29, 730 (1955) [English trans1.: Soviet Phys.-JETP 2, 636 (1956)].

25. R. B. Dingle, Proc. Roy. Soc. (London) A211, 517 (1952).

26. A. V. Gold, in Solid State Physics, edited by J. F. Cochran and R. R. Haering (Gordon and Breach Science Publishers, Inc., New York, 1968), Vol. 1, p. 39.

27. D. Shoenberg, in Progress in Low Temperature Physics, edited by C. J. Gorter (North-Holland Publishing Co., Amsterdam, 1957), Vol. 2 , p. 226.

28. J. R. Anderson, Ph.D. Thesis, lowa State University, 1962 (unpublished).

29. P. T. Panousis, Ph.D. Thesis, lowa State University, 1967 (unpublished).

30. C. P. Bean, R. W. DeBlois, and L. B. Nesbitt, J. Appl. Phys. 30, 1976 (1959).

31. P. T. Panousis and A. V. Gold, Rev. of Sci. Instruments 40,120 (1969).

32. Wo J. O'Sullivan and J. E. Schirber, Cryogenics I, 118 (1967).

33. R. A. Phillips, Southern Missouri.State College, private communication, 1971 .

34. R. L. Aurbach, J. B. Ketterson, F。 M. Mueller, and L. R. Windmiller, in The Inversion of de Haas-van Alphen Data: 1. Closed Surfaces with Inversion Symmetry. (Argonne Nat. Lab. Report ANL-7659, Argonne, iा1., 1970).

35. F. M. Mueller, Phys. Rev. 148, 636 (1966).

36. R. F. Girvan, Boston College, private communication, 1971 .

37. F. M. Mueller and M. G. Priestly, Phys. Rev. 148, 638 (1966). 


\section{ACKNOWLEDGEMENTS}

The author wishes to thank his major professor, John L. Stanford, for his suggestions, helpful discussions, and encouragement during this investigation and for his concern for the well-being of each member of his group. The members of the Stanford research group have been helpful and willing to listen.

The author is also indebted to Dr. R. A. Phillips for discussions on the finer details of the dHvA effect and the pulsed-field apparatus, as well as for the interpretation of the tungsten results. The assistance of former members of Dr. A. V. Gold's research group who contributed to the design of the experimental apparatus is acknowledged. Without the digital equipment developed by Dr. T. P. Panousis, experimental results of this accuracy would have been almost impossible to obtain. it is a pleasure to thank Professors S. H. Liu, L. Hodges, and D. Pursey for many helpful discussions on the theoretical aspects of this investigation.

To my wife, Bea, go special thanks for her patience, understanding, and constant encouragement during these past years of study and research. The secretarial skills of Mrs. Carol Kline are appreciated in the typing of the manuscript. 
VII. APPENDIX A

\section{COEFFICIENTS FROM INVERSION SCHEME FITS}

HOLE OCTAHEDRON

$\begin{array}{ccc}\# & J & \begin{array}{c}\text { RADIUS* } \\ \text { COEF }\end{array} \\ & & \\ 1 & 0 & 0.131200003 \\ 2 & 4 & 0.300859002 \\ 3 & 6 & -0.144265001 \\ 4 & 8 & 0.940868001 \\ 5 & 10 & -0.387562000 \\ 6 & 12 & 0.242699001 \\ 7 & 12 & 0.128054000 \\ 8 & 14 & -0.6439530-01 \\ 9 & 16 & 0.161086001 \\ 10 & 16 & 0.267312000 \\ 11 & 18 & 0.127289000 \\ 12 & 18 & 0.644677000 \\ 13 & 20 & 0.447419000 \\ 14 & 20 & 0.393513000 \\ 15 & 22 & -0.155024000 \\ 16 & 22 & 0.275068000 \\ 17 & 24 & 0.797590000 \\ 18 & 24 & -0.198070000 \\ 19 & 24 & 0.187362000\end{array}$

\section{ELECTRON LENS}

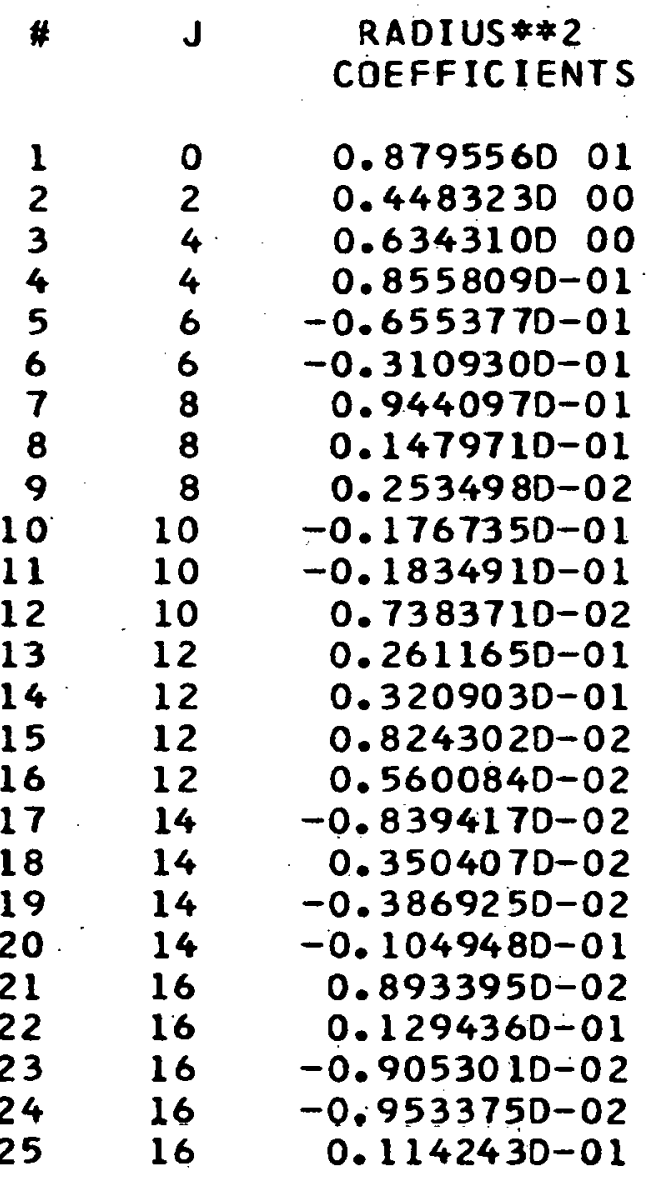

GAMMA $=1.6078$ 
HOLE ELLIPSOID

$\begin{array}{rr}\text { \# } & \text { J } \\ & \\ 1 & \\ 2 & 0 \\ 3 & 2 \\ 4 & 2 \\ 5 & 4 \\ 6 & 4 \\ 7 & 4 \\ 8 & 6 \\ 9 & 6 \\ 10 & 6 \\ 11 & 6 \\ 12 & 8 \\ 13 & 8 \\ 14 & 8 \\ 15 & 8 \\ 16 & 10 \\ 17 & 10 \\ 18 & 10 \\ 19 & 10 \\ 20 & 10 \\ 21 & 10 \\ 22 & 12 \\ & \end{array}$

\section{RADIUS $* 2$ COEFFICI ENTS}

$0.3528140 \quad 02$

$-0.8318530-02$

$-0.597569000$

0.210776000

0.102084001

0.266903000

$0.1247220-02$

$-0.6776950-01$

$-0.7651980-01$

$0.5822000-02$

$0.4492170-01$

$0.256474 D-01$

$0.5237700-01$

$0.2310100-02$

$0.1784030-01$

$0.1532800-01$

$0.9287760-02$

$-0.1132440-01$

$0.175208 D-01$

$0.3763650-04$

$0.353431 D-01$

$0.605098 D-01$

\section{$\#$

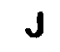 \\ RADIUS **2 COEFFICIENTS}

$23 \quad 12 \quad-0.1859230-01$

$24 \quad 12$

$25 \quad 12$

$26 \quad 12$

$27 \quad 12$

$28 \quad 12$

$29 \quad 14$

$30 \quad 14$

$31 \quad 14$

32

33

34

35

36

37

38

39

40

41

42

43

44

45
$-0.6649740-01$

0.1466700-01

$-0.5174170-01$

$-0.6018350-02$

$0.1268780-01$

$0.4813400-01$

$-0.6223770-02$

$-0.4132820-01$

$0.3114650-01$

$0.2541590-01$

$-0.2756810-01$

$-0.2596160-01$

$-0.1786960-01$

$0.1748390-01$

$-0.223194 D-01$

$-0.4286940-01$

$0.174048 \mathrm{D}-01$

$-0.7478760-02$

$-0.2238790-01$

$-0.300702 D-01$

$0.3235280-01$

$0.9382680-02$

ALPHA $=1.3904$

GAMMA $=0.8638$ 
VIII. APPENDIX B

FREQUENCY ANALYSIS PROGRAM LISTIING AND DISCUSSION

This appendix describes the computer programs used to transfer and analyze the dHvA data. Job A334TTT, "TAPE7 TO TAPE9," transfers the data from a 7-track magnetic tape produced from the punched paper tape output created at the time the experimental equipment was being operated to a 9-track tape for data analysis and storage. Job B334FFT is the program responsible for the data analysis. Both of these listings appear after the discussion part of this appendix.

In "TAPE7 TO TAPEg" the assembler language routine CHGTC takes the intermixed dHvA signal and magnetic field value arrays which are input as 4096 characters in the array $Y$, and maps these characters in a $1: 1$ fashion to a new character set which is input for the assembler language routine TRANS. TRANS decodes the $Y$ character array and returns an array $Y$ containing 2008 data points and an array $H$ containing 20 values for the magnetic field. The field values are then converted from integer values ranging from 0 to 4095 to actual readings in megagauss through the use of the constants set at the time the experiment was conducted. An identification card, the $Y$ and $H$ arrays, and the experimental constant data are recorded on 9-track tape as pairs of records for each data set to be read later hy the data analysis program. Appropriate labels and information about the magnetic field values are printed to enable one to determine whether the data set has been converted and stored correctly. A plot of typical data for an angle of $28.9^{\circ}$ from [010] in the (001) plane is shown in Figure 22. The $Y$ array of integer values (up to \pm 128 ) of the dHvA 


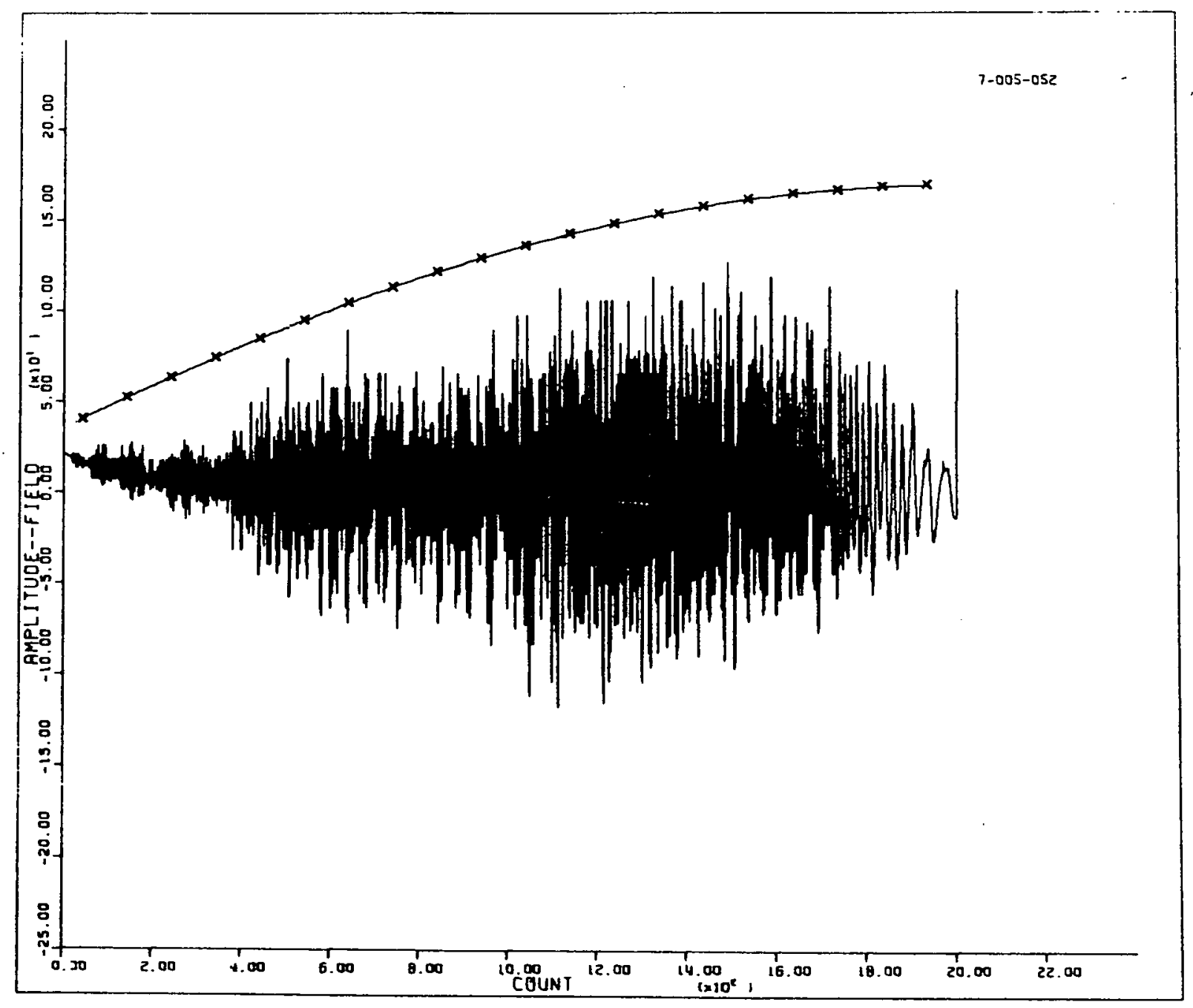

ร

Figure 22. A plot of typical dHvA data. This data was taken at $28.9^{\circ}$ from [010] in the (001)
plane 
signal was plotted versus integer count of time in units of $4 \mu \mathrm{sec}$. The $H$ array of field values is in units of megagauss for the same integer time count.

The analysis program B $334 \mathrm{FFT}$ used the fast-Fourier subroutine RHARM from the IBM Scientific Subroutine Package. This analysis is $10-60$ times faster than the filter-periodogram technique described by Panousis (29) with comparable frequency accuracy and resolution. Figure 23 shows a filter-periodogram frequency analysis of dHvA data taken at $28.9^{\circ}$ from [010] in the (001) plane and shown in Figure 22. Figure 24 shows the frequency analysis of the same data set by the fast-Fourier technique. The peaks labelled $\beta 1, \beta 2, \beta 3$ are plotted as points labelled $\beta$ for frequencies at $28.9^{\circ}$ from [010] in the $(001)$ plane as shown in Figure 6 . Peaks labelled with subscripts in Figure 24 (such as $\left(\mathrm{Bl}_{2}\right)_{2}$ ) are second harmonics, and peaks with two frequencies (such as $\gamma \pm \pi$ ) are sums and differences of the appropriate fundamental terms. Only frequencies identified as fundamental were plotted in Figure 6 . In both Figures 23 and 24 the graphs are composites of several small-range frequency scans, since each program does its best on frequency scans of one octave or less. For purposes of comparison the frequency ranges and selected data ranges used were identical for both analyses. The computer time used to obtain the filterperiodogram frequency results for the data set was around 35 minutes of CPU time, while the same frequency results were obtained by B334FFT with about 45 seconds of CPU time. From the 27 peaks identified in Figure 24 we get some feeling of the power of either analysis program to extract many frequencies with high accuracy and resolution from a single data set. 


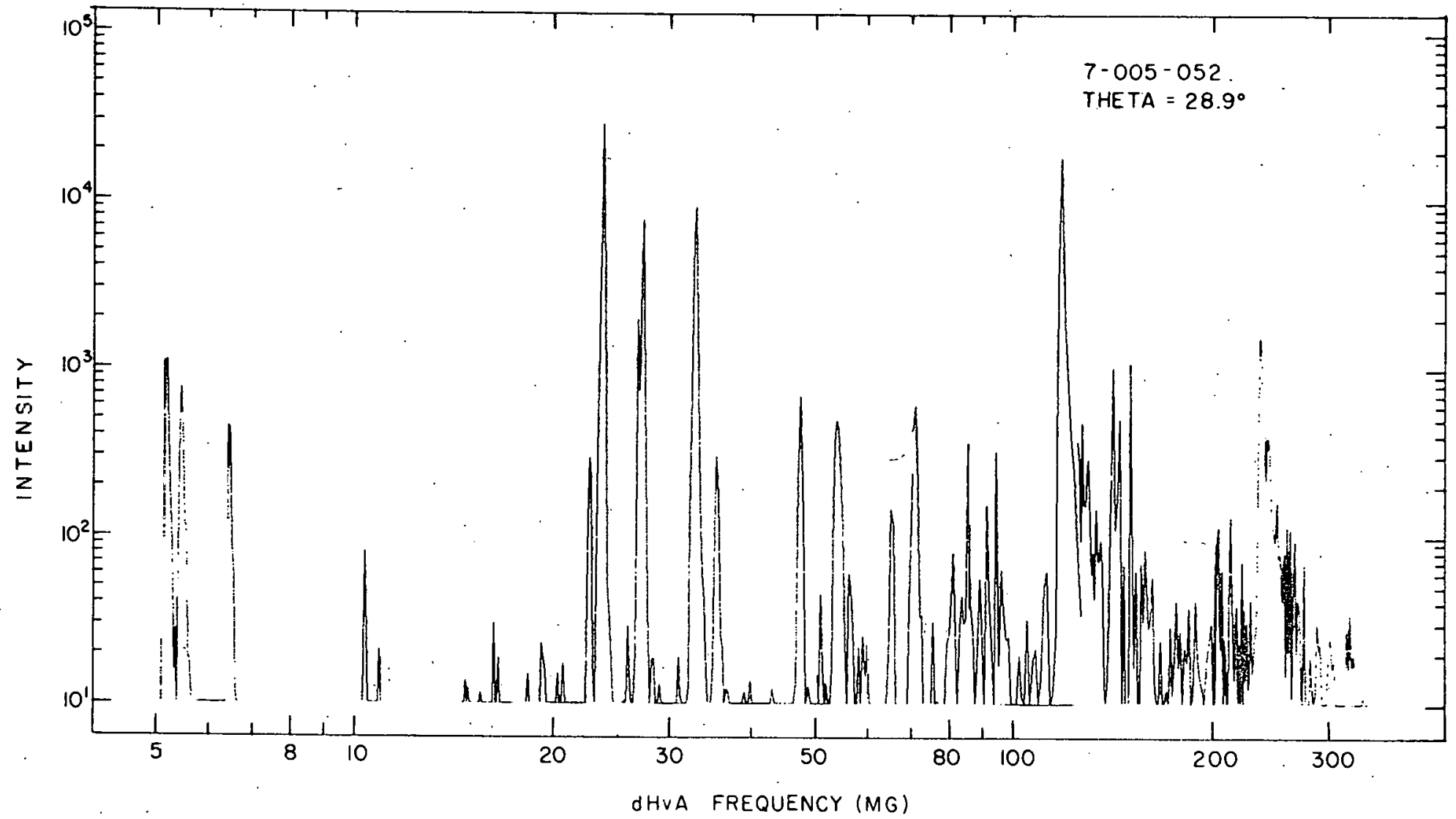

$\infty$

Figure 23. A filter-periodogram frequency analysis of the dHvA data shown in Figure 22 


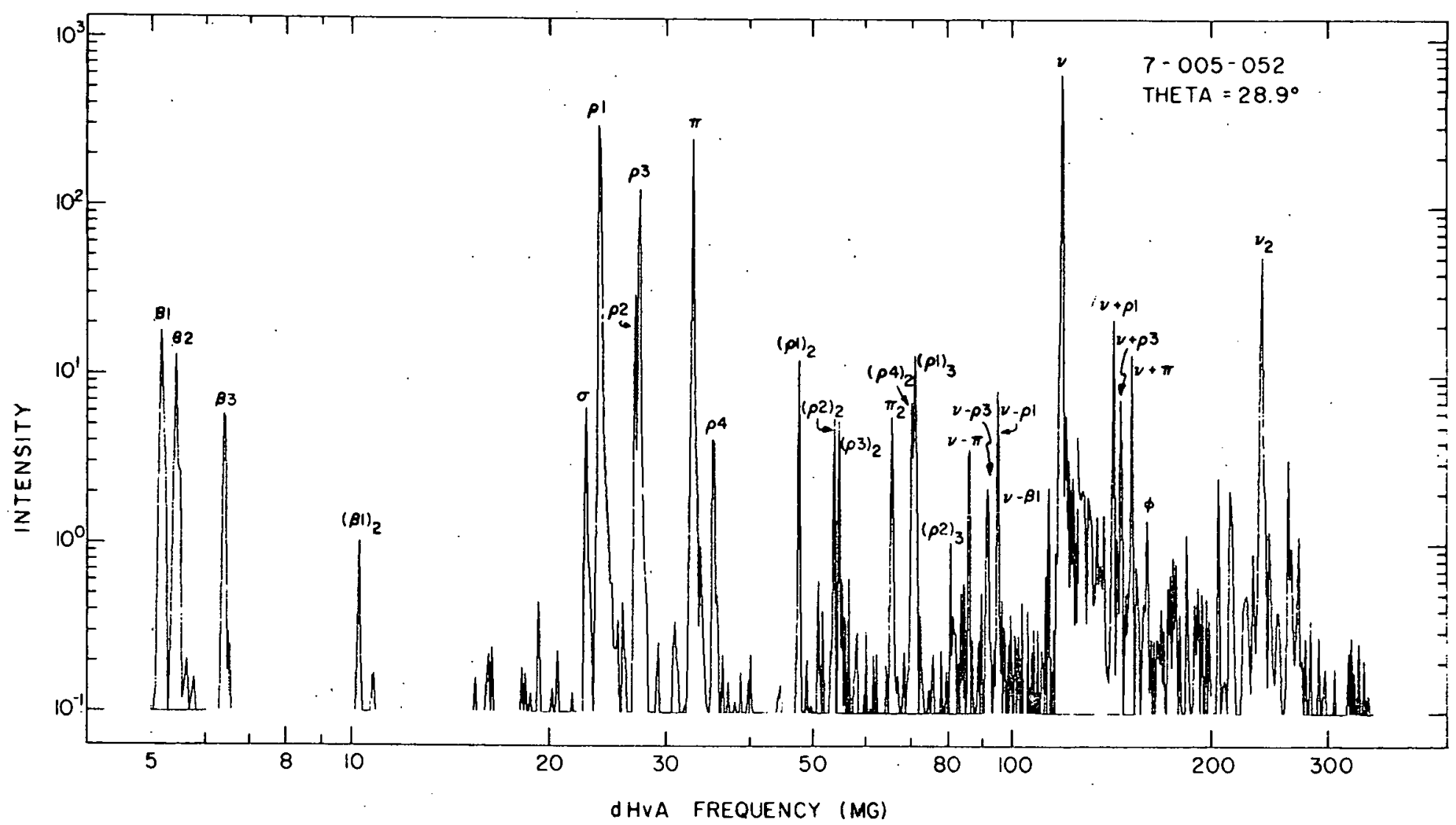

क̊

Figure 24. A fast-Fourier frequency analysis of the dHvA data shown in Figure 22. The fundamental frequencies from this analysis are plotted and labelled in Figure 6 at $28.9^{\circ}$ from [010] in the $(001)$ plane 
The intensity scales on Figures 23 and 24 should not be compared for absolute intensity.

B334FFT was written with several options to handle the most frequently desired modes of data input and analysis. It assumes that the data is identified by the same cards used with A334TTT to create the 9track data storage tape. Some named COMMON storage areas are used to pass different variables at different stages in the calculation and/or used for work space to keep storage space to a minimum. The program will run in either a $(160 \mathrm{~K}, 32 \mathrm{~K})$. region or $(96 \mathrm{~K}, 96 \mathrm{~K})$ region of fast-core and bulk-core storage depending upon how the HIARCHY card is set up in the LKED step of the executed procedure FORTH.

All reading of tapes and cards is done in subroutine READIN. BPASS is equal to 0.0 for the first call of this subroutine for initialization of graph labels and program options. A set of frequency cards is read in and stored, a blank card is detected, followed by the first data identification (ID) card which is read. The appropriate data set is obtained from a sequential tape and control is returned to MAIN. Each further call to READIN reads an 10 card and provides an additional data set for analysis. If a blank card is encountered, a new set of frequencies, one blank card, and one ID card are again read in before control is returned to MAIN.

Subroutine BFIELD corrects for the time delay effects described by Equation.4. For all of this investigation TZERO $=40 \mu \mathrm{sec}$ was found appropriate to obtain approximately equal frequency results for both "rising field" and "falling field" data. Since molybdenum is not ferromagnetic, 
$H Z E R O=0.0$ for this work.

Subroutine FITH identifies the type of field contained in the data as "rising" and/or "falling", checks that the number of data points associated with each identification is acceptable, and returns the code of the type of field detected, the appropriate calculated array of up to 2008 field values, and the limits on the array.

Subroutine RANGE takes into account the fact that the amplifiers in our apparatus are capable of passing time frequencies in only a certain given range $(\sim 5-100 \mathrm{kHz})$, and that our dHvA digital equipment is capable of taking data at equal time intervals of $4 \mu \mathrm{sec}$ minimum (i.e., only two points per cycle are obtained for a $125 \mathrm{kHz}$ signal). Since dHVA oscillations are periodic in $I / H$ rather than in real time, for a given pair of data points there is a minimum and a maximum value for possible dHvA frequencies contained in the data, as given by the relation

$$
\text { time freq. } \doteq\left|\frac{\Delta\left(\frac{l}{H}\right)}{\Delta T}\right| \text { dHvA freq. }
$$

with $\Delta T$ and $\Delta H$ computed from this pair of points and the time frequency being one of the amplifier frequency limits. The upper amplifier limit assures us of taking data at a rate of more than two points per cycle. We have found that when data is analyzed for a given dHvA frequency using only those data points which lie within the time frequency limitations given above, we obtain the best analysis.

This subroutine works best if the desired frequency ranges are input in increasing order, and computes starting and stopping counts for analysis of the data for each of the frequency ranges it is given. The 
routine is bypassed by the MAIN program if the starting and stopping count values are overridden from data input cards.

Subroutine GENY converts a portion of the dHVA data array YI, taken in equal increments of time, to an expanded array $Y$ of 16,384 data points computed at equal increments of $1 / H$, since the dHvA data is periodic in $1 / H$ rather than in time, by using the inverted field values in the $\mathrm{HI}$ array. The number of data points used in the conversion depends upon NSTART (1) and NSTOP (1) as usually determined by the RANGE subroutine for the 1 th frequency range. Straight-line interpolation is used between data in both the $\mathrm{YI}$ and $\mathrm{HI}$ arrays and is generally a good approximation. Trying to increase the resolution in the data by setting RES $\neq 0$ on input cards and filling part of the large $Y$ array with zeroes was not found to be a satisfactory means of increasing the resolution. It should be noted that this mapping from real time space to $1 / H$ space causes time-periodic noise signals to be transformed into random noise in $1 / H$ space.

Subroutine OUTPUT provides printed output of the frequency analysis and optional tape records and/or graphic display of the results. Tape output was used to input frequency results for computer averaging by another program. If the frequency range desired contains more than 300 possible output frequencies, the output is broken down into scans which do not overflow the output data arrays. Subroutine PEAKS is called by OUTPUT to search for peaks in the frequency spectrum and to fit a second order curve to determine the true peak frequency and its intensity. These frequencies are listed by OUTPUT if their intensities are greater than .005 or any value set by the override variable ALIM. 
The MAIN routine sets up the actual frequency ranges to be analyzed and controls the appropriate choices of subroutines for each particular analysis, depending on information generated from the previous analysis. The original data set is stored and restored, if needed, by this routine.

The IBM subroutine RHARM analyzes $N=2 * 2^{M}$ data points and returns $N+2$ Fourier coefficients corresponding to the constant coefficient and the sine and cosine coefficients on the first $2^{M}$ harmonics in the equation

$x_{j}=\frac{a_{0}}{2}+\left(b_{0}=0\right)+\sum_{k=1}^{N-1}\left(a_{k} \cos \left(\frac{\pi j k}{N}\right)+b_{k} \sin \left(\frac{\pi j k}{N}\right)\right)+\frac{a_{N}}{2}(-1)^{j}+\left(b_{N}=0\right)$.

Test data run with this subroutine has led us to conclude that:

(1) RHARM is capable of determining a single sine-wave frequency to the nearest integer $k \pm 0.5$.

(2) Resolution of frequencies less than 3 cycles apart is generally not possible.

(3) The frequency determined is not extremely dependent on the shape of a long-wave envelope containing the sine wave.

(4) The frequency determined by RHARM does not appear to depend on the value of $M$, as long as the frequency determined is not approaching the highest frequency capable of being determined with a given $M$.

(5) Phasse shifting part of a wave train with respect to another part with identical frequency does cause severe problems in frequency determination. Many harmonics are necessary to reproduce the wave-form created, and the fundamental frequency in the data may 
be smeared or be lost all together.

The intensity for the $k^{\text {th }}$ harmonic in Equation 8 is computed from the $Y$ array of returned coefficients of RHARM by using

$$
\text { intensity }=\left(a_{k}\right)^{2}+\left(b_{k}\right)^{2}=(Y(n))^{2}+(Y(n+1))^{2}
$$

for the frequency (in megagauss)

$$
\text { frequency }=\frac{k}{\left|\frac{1}{H_{1}}-\frac{1}{H_{2}}\right|}=\frac{n-1}{2} \cdot \frac{1}{\left|\frac{1}{H_{1}}-\frac{1}{H_{2}}\right|}
$$

where $n=2 k+I$ is the index on the $Y$ array. Note that $n$ is always odd. If this is not true, incorrect pairs of coefficients from two different harmonics would be used in computing the intensity with incorrect results. A listing of the programs and some comments on the called library subroutines follows. 


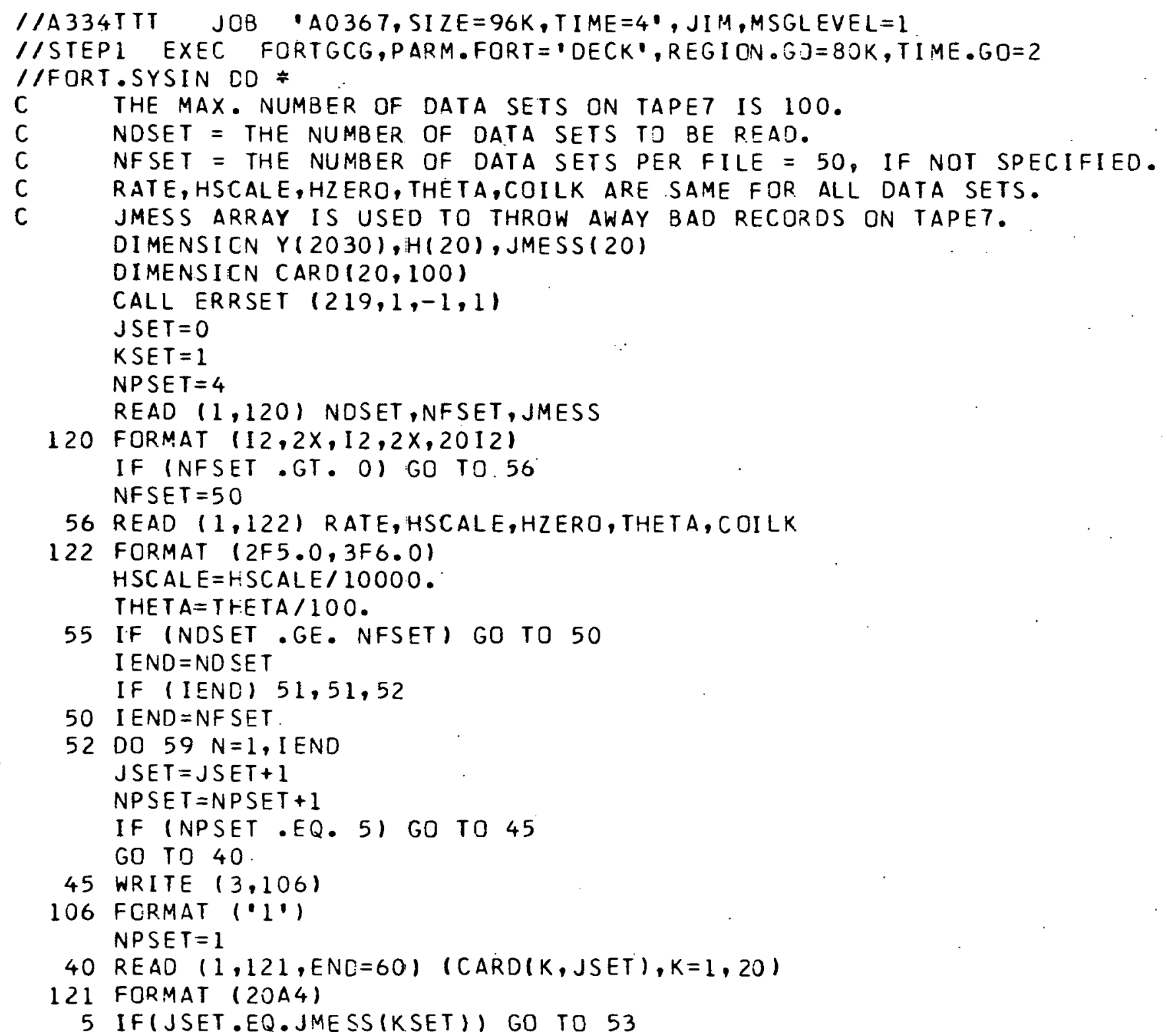

56 READ $(1,122)$ RATE, HSCALE, HZERO, THETA, COILK

122 FORMAT $(2 F 5.0,3 F 6.0)$ HSCALE $=H S C A L E / 10000$. THE TA = THETA $/ 100$.

55 IF (NDSET. GE. NFSET) GO TO 50 I END $=$ NDSET

5 IF (JSET.EQ.JMESS(KSET) GO TO 53 
$1 \operatorname{READ}(10,110, E R R=1000, E N D=57) \quad(Y(I), 1=1,1024)$

110 FORMAT (32(32A4))

GO TO 3

1000 WRITE $(3,111)$

11 .1 FORMAT 1 ERROR IN DATA TRANSFER FROM TAPET')

CARD $(10, J S E T)=1111$

READ $(10,110) \quad(Y(1), I=1,1024)$

3 CALL CHGTC $(Y)$

CALL TRANS $(Y, H)$

WRITE $(3,125)$ (CAF.D(K, JSET), $K=1,20)$

125 FORMAT $(' 0,, 20 A 4)$

WRITE $(3,123)$ RATE, HSCALE, HZERO, THETA, COILK

123 FCRMAT 100 RATE $=1, F 4.0,1$ KCPS. HSCALE=1,F6.4, $\quad$ HZERD $=1, F 6.0$

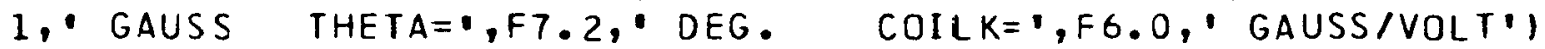

WRITE (.S) (CARD(K, JSET), K=1,20)

WRITE $(3,107)$

107 FORMAT $(10$ THE FIELD VALUES ARE: $1 / 1$

WRITE $(3,103) \quad(H(I), I=1,20)$

103 FORMAT $(10 F 12.2)$

DO $6 \mathrm{~J}=1,20$

$6 H(J)=H(J) *(10.14096) * C O I L. K / H S C A L E$

WRITE (S) (Y(I) I I =1,2008), (H(I), I =1,20), RATE,HSCALE,HZERO,

ITHETA,CCILK.

WRITE $(3,108)$

108 FCRMAT (')

WRITE $(3,103) \quad(H(1), I=1,20)$

WRITE $(3,104)$

104 FCRMAT $(\cdot 0 \cdot)$

59 CONT INUE

END FILE 9

NDSET $=$ NDSET - NFSET

GO TO 55

60 WRITE $(3,130)$

130 FORMAT 1.0 YOU' RE CUT OF CARDS, BUDDY 1

GO TO 58

57 WRITE $(3,101)$

101 FORMAT. 1 "O CAN'T YOU EVEN COUNT--STUPID' 


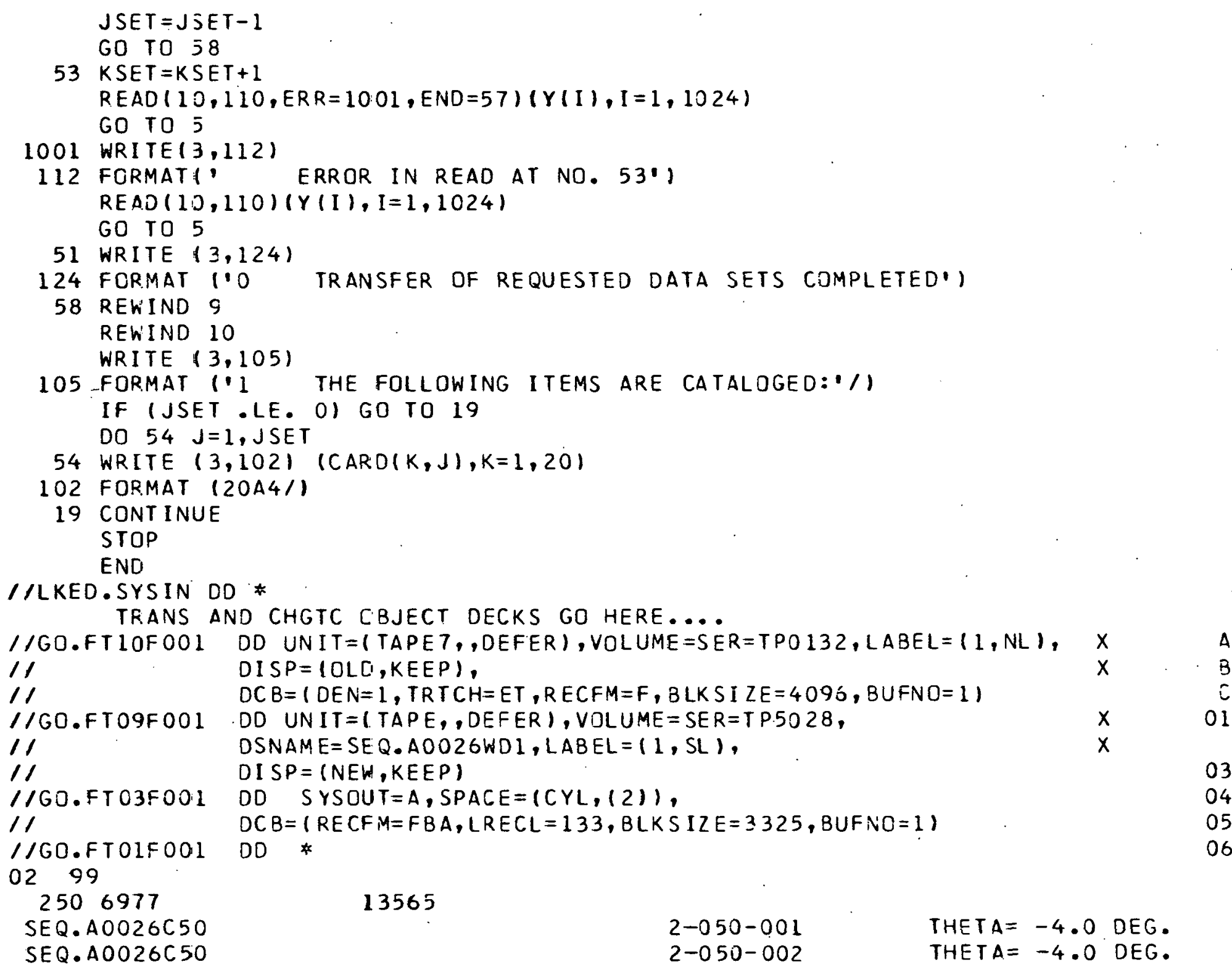


$/ / B 334 \mathrm{FFT} J 08 \cdot A 0367, \mathrm{SI}=224 \mathrm{~K}, \mathrm{TI}=11, \mathrm{LI}=6 \cdot, \mathrm{JIM}, \mathrm{MSGL} \subseteq V E L=1$

$/ / S T E P 1$ EXEC FORTH,PARM. : OORT = 'MAP, DECK, OPT $=2, L I S T, X R E F '$,

$1 /$ REGION.FORT $=224 \mathrm{~K}$, PARM. LKED = 'MAP,LIST,LET, HI AR',

$1 /$ TIME.GO $=8, R E G I C N, G D=(160 K, 32 \mathrm{~K}), L I N E S=6$

//FORT.SYSUT2 DD UNIT $=$ SPOOL, SPACE $=(C Y L,(1,1))$

I/FORT.SYSIN CD*

C

C

C

DE HAAS-VAN ALPHEN FREQUENCY COMPUTATIONS

\#** THIS IS THE MAIN PROGRAM * *

THIS PREGRAM CONTAINS THE FAST FOURIER FIT SUBROUTINE RHARM.

CCMMON/ AUTOF/FF(20), FL (20), NSTART(20), NSTOP(20)

CCMMON/CALC/S(2048), INV(2048)

COMMON/DATA/H2OS $(20), Y 1 S(2008), H I S(2008)$

CCMMON/FIELD/OT, TZERO, THETA,HZERO,H2O( 20$)$

COMMON/FREQ/FFIRST $(10), F L A S T(10)$, TZERO 1110$)$

COMMON/GIANT/Y (16388)

COMMON/LABS/GLAB 1 (5), GLAB2 (5), XLA31(5), YLAB 1(5), DDATE (7),

$1 R L A B(5), F L A B(5)$

DIMENSICN H(2008), HI (2008), YI(2008)

EQUIVALENCE $(H(1), H I(1), S(1)),(Y 1), S(2009))$

BPASS $=0.0$

$O T M R=0.0$

$O T M C=0 . C$

$M M M=13$

$M M=16334$

424 CALL READIN IBPASS, OMEGAL, OMEGA2, STEP, STEPO, TMR, TMC, RES, MFSET,

IRATE, HSCALE, THETAI, COILK, NGRAPH, MSTART, MSTOP, ALIM, NTOUT, NSERI $K F=1$

22 TOOO=TZEROI (KF)

$T Z E R O=T 000 /(1 . E 06)$

DC $30 \quad I=1,20$

$30 H 20(I)=H 2 O S(I)$

CALL BFIELD

CALL FITH (IFIELD,LRISE, LFALL)

DO $70 \quad I=1,2008$

$H I(I)=1 . / H(I)$ 
$70 \mathrm{HIS}(\mathrm{I})=\mathrm{HI}(\mathrm{I})$

$J=1$

$6 F F(J)=F F I R S T(K F)$

$1 F L(J)=S T E P$ SFF $(J)$

IF (FL(J) . GE. FLAST(KF)) GO TO 2 $\mathrm{JJ}=\mathrm{J}+1$

$F F(J J)=S T E P O F F(J)$

$\mathrm{J}=\mathrm{J} \mathrm{J}$

GO TO 1

$2 F L \mid J)=F L A S T(K F)$

IF (KF. GE. MFSET) GO.TO 3

$K K F=K F+1$

IF (TZEROL (KKF) .NE. TOOO) GO TO 3

$J=J+1$

$K F=K K F$

GO TO 6

3 IF (IFIELD .GT. 2) GO TO 4 $M F F C T=J$

NFTOT $=J$

IF (IFIELD .LT. 2) GO TO 5

DO $10 \quad I=1, M F F C T$

$J J=I+M F F C T$

$F F(J J)=F F(I)$

$10 \mathrm{FL}(J J)=F L(I)$

$N F I C T=M F F C T+1$

NFFCT $=2 * M F F C T$

NFTOT $=$ NFFC T

GO TO 5

4 NFICT $=1$

NFFCT $=\mathrm{J}$

NFTOT $=J$

5 IF (NFTOT.GT. 20 ) GO TO 26

IF (IFIELD-2) $39,41,40$

RISING FIELD ONLY.

39 LORI SE = MSTART

IF (MSTOP . GE. LRISEI GO TO 50

LRI SE $=$ MSTOP 


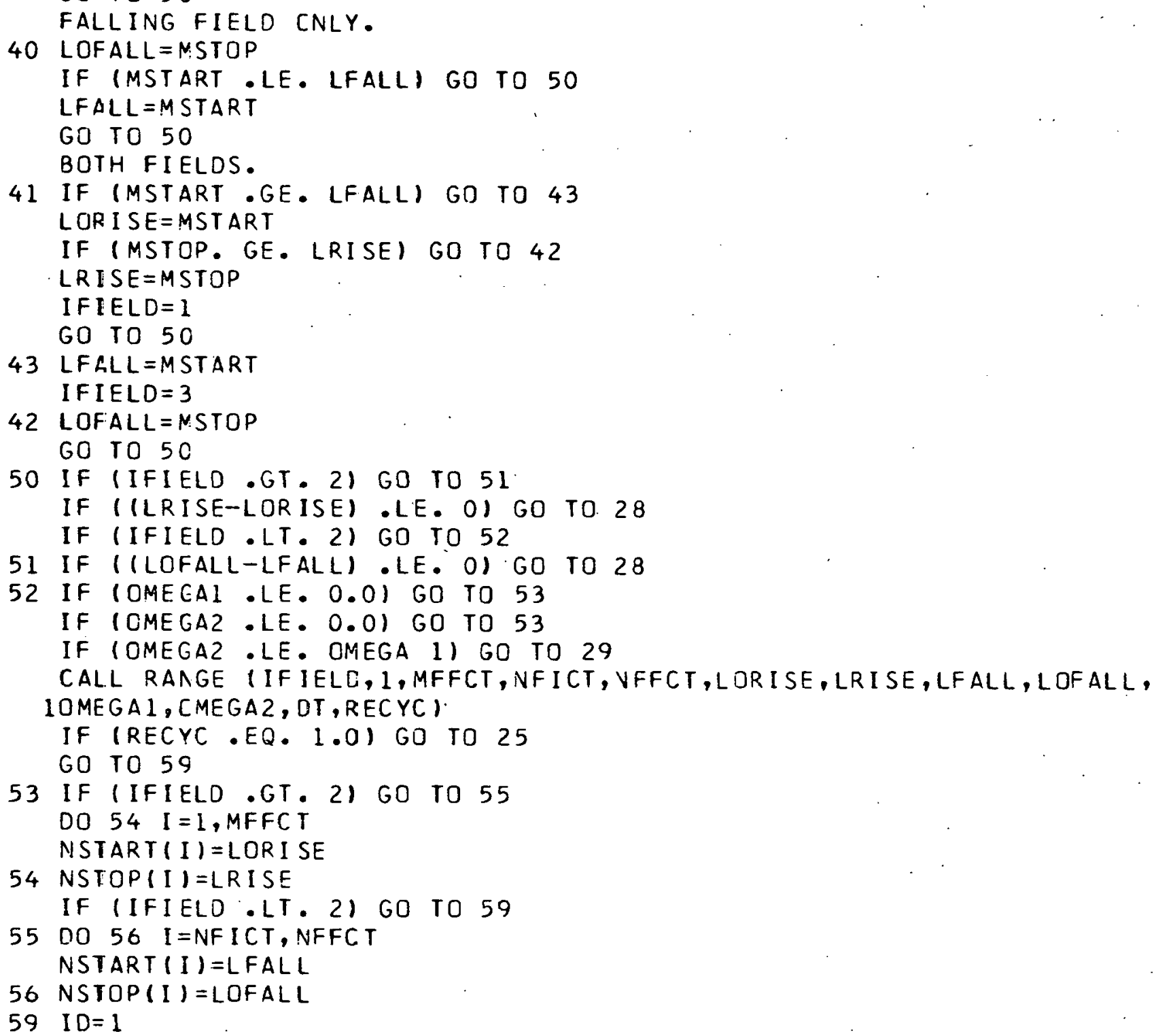




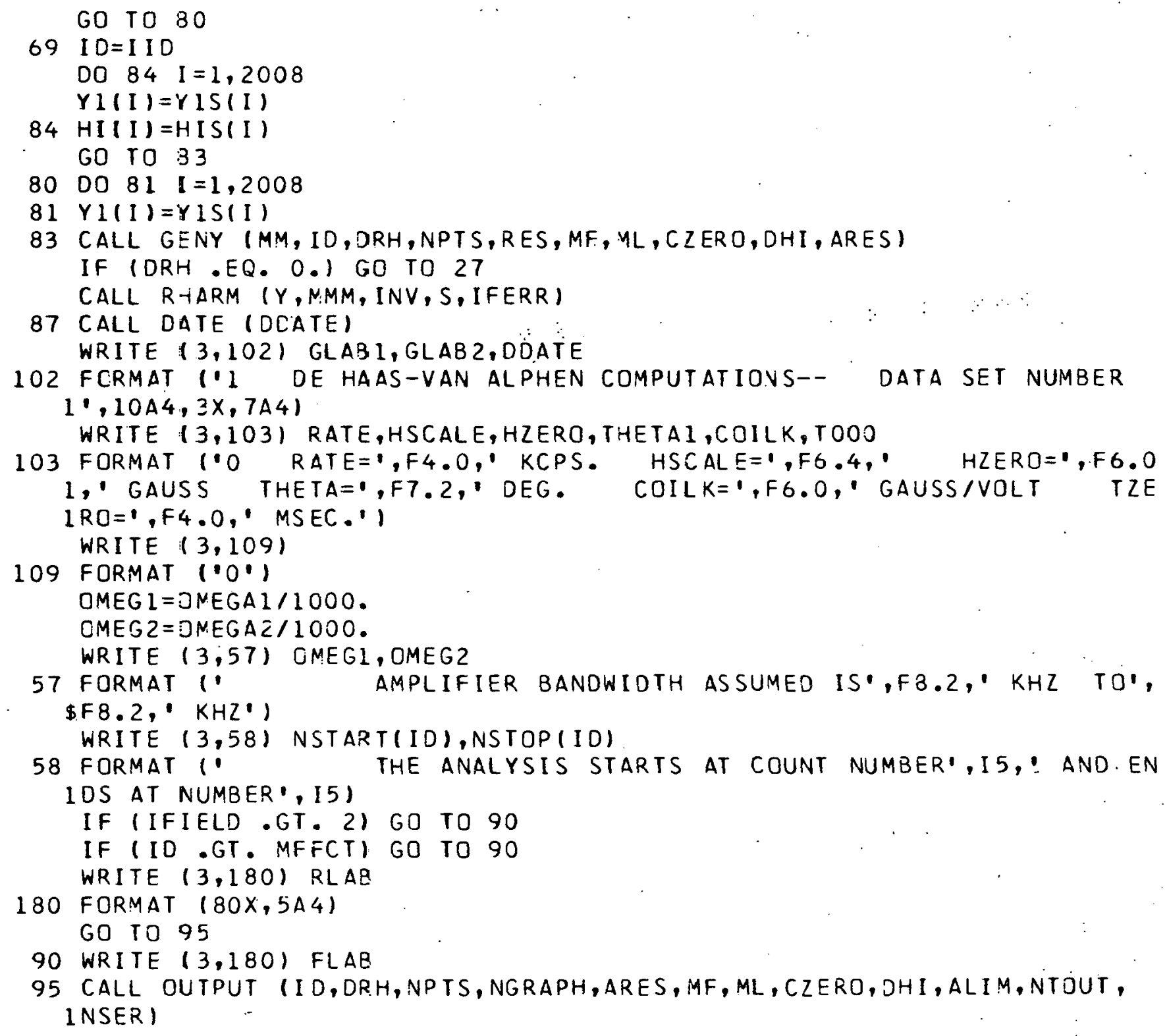







SUBROUT INE READIN IBPASS, OMEGA 1, OMEGA2, STEP, STEPO, TMR, TMC, RES, 1 MFSET, RATE, HSC ALE, THETA1, COILK, NGRAPH, MSTART, MSTOP, ALIM, NTOUT, (NSER )

THIS SUBROUTINE READS DATA FROM 9 TRACK TAPE.

THIS SUBROUTINE READS ALL CARD DATA FOR THIS PROGRAM.

A. BLANK CARD INDICATES END OF FREQ. SET, OR SETS OF DATA.

MAX. OF NINE FREQ CARDS IN A SET.

CCMMON/CALC/TCARD(10)

CCMMGN/CATA/HZOS $(\bar{Z} 0), Y 1 S(2008)$

COMMON/FIELD/OT, TZERO, THETA, HZERO, H2O(20)

COMMCN/FREQ/FFIRST (10), FLAST (10), TZERO1(10)

COMMON/LABS/GLAB 1 15$)$, GLAB2 $(5), X L A B 1(5), Y L A B 1(5)$, ODATE $(7)$,

$1 R L A B(5), F L A B(5)$

CALL ERRSET $(219,1,-1,1)$

REAL 4 BLANK $/$.

$P I E=3.14159$

IF (BPASS .GT. 0.0 ) GO TO 40

READ $(1,101) \times L A B 1, Y L A B 1, R L A B, F L A B$

101 FORMAT (20A4)

READ $(1,110)$ EXTIME, OMEGA1, OMEGA2, STEP, STEPO, NGRAPH, NTOUT, NSER, IALIM

110 FORMAT (F5.1,2E10.2, 2F5.2,3I 5,F5.2)

IF (EXTIME.GT. 0.0$)$ GO TO 80

EXTIME $=10.0$

$80 E X T I M E=E X T I M E * 60.0$

$T M R=0.0$

$T M C=0.0$

CALL STARTM (TMR, TMC)

$B F R E Q=0.0$

$B P A S S=1.0$

$71 K=1$

85 READ $(1,105, E N D=5)$ FFIRST $(K), F L A S T(K), T Z E R J 1(K), R E S, M S T A R T, M S T O P$ NOTE: RES, MSTART AND MSTOP MUST BE READ OFF THE 'BLANK' CARD

C SEPARATING FREQUENCY CARDS FROM DATA CARDS IF ANY VALUES ARE

C $\triangle R E$ TO RE RETAINED. 
105 FORMAT (2E 10.2,F5.0,F5.3,2I 5 )

IF (FFIRST(K) LLE. 0.0$)$ GO TO 11

IF (FLAST(K) . LE. FFIRST(K)) GO TO 12

$K=K+1$

GO TO 85

11 MFSET $=K-1$

IF (MSTART . NE: 0.0$)$ GO TO 20

MSTART $=1$

20 IF (MSTOP. .NE. 0.0$)$ GO TO 40

MSTOP $=2008$

40 CALL STCPTM (TMR, TMC)

IF (TMC .GT. EXTIME) GO TO 82

CALL STARTM (TMR, TMC)

86 READ $(1,106, E N C=5)$ TEND, CARDR, CARD

106 FORMAT $(20 X, A 4,20 X, 2 A 4)$

IF (TEND.EQ. BLAPJK) GO TO 50

REWIND 9

GO TO 86

50 IF (CARDR . EQ. BLANK) GO TO 71

70 READ $(9, E R R=1001, E N D=60)$ TCARD, GLAB 1, GLAB2

IF (GLAEI(2) . EQ. CARDR) GO TO 51

READ $(9, E N D=65)$

GO TO $7 \mathrm{C}$

51 IF (GLABI(3) EQ . CARD) GO TO 52

READ $(9, E N O=65)$

GO TO $7 \mathrm{C}$

52 READ (9,ERR $=1002, E N D=61)$ YIS,H20S, ZATE,HSCALE, HZERO, THETA1, COILK THETA=THETA 1 *PIE/ 180 .

IF IRATE .LE. 0.0; GO TO 7

DT $=1 . /($ RATE*1000.1

RETURN

7 WRITE $(3,120)$

120 FORMAT ('O PROSRAM TERMINATED--RATE NOT . GT. ZERO') GO TO 84

12 W'RITE $(3,107)$

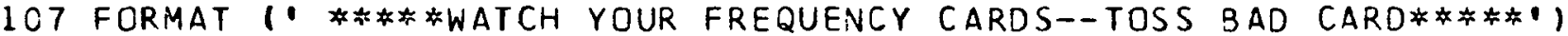
GO TO 85 


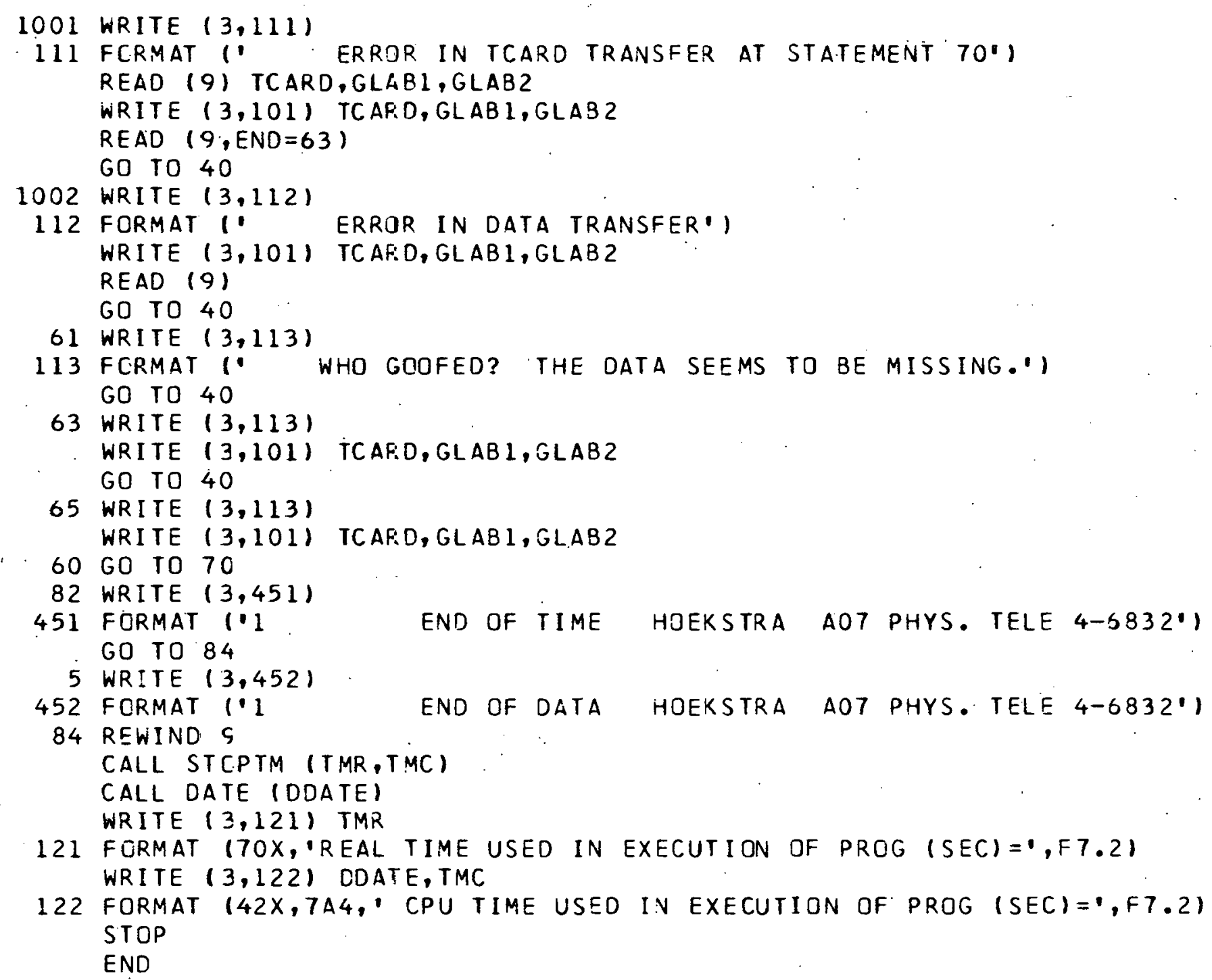


this SUBroutine calculates the 'B' fielo from the 20 'H' VAlues. IT WILL CORRECT FOR BOTH TIME DELAY AND DEYAGNETIZING EFFECTS.

\section{COMMON/CALC/X1 $(20), W(20)$}

COMMON/FIELD/DT, TZERO, THETA,HZERO,H2O(20)

DOUBLE PRECISION Q(6)

$X 1(1)=42$.

$W(1)=1$.

DO $1 \quad I=2,20$

$x(1)=x i(I-1)+100$.

$1 W(I)=1$.

CALL OPLSPA $(5,20, \times 1, H 20, W, Q, 0.0)$

DO $2 \mathrm{I}=1,20$

C

CALCULATE DERIVATIVE D=DH/DT

$X=X 1(I)$

$K=2$

$D=Q(2)$

$9 D=0+K * Q(K+1) * X$

IF $(K \cdot G E \cdot 5)$ GO TO 2

$K=K+1$

$X=X * X 1(I)$

GO 109

$2 \mathrm{H} 2 \mathrm{O}(\mathrm{I})=\mathrm{H} 2 \mathrm{O}(\mathrm{I})-\mathrm{D} * \mathrm{TZERO} / \mathrm{DT}$

IF (HZERO . LE. 0.0$)$ GO TO 4

$P=(\operatorname{COS}(T H E T A)) * \neq 2$

$X=H Z E R O *(3 . * P-1.1 / 2$.

$Y=H Z E R O * P$

DO $3 \quad I=1,20$

$3 \mathrm{H} 2 \mathrm{O}(\mathrm{I})=\mathrm{HZERO}+\mathrm{H} 2 \mathrm{O}(1) *(\mathrm{H} 2 \mathrm{O}(\mathrm{I})+\mathrm{X}) /(\mathrm{H} 2 \mathrm{O}(1)+Y)$

4 RETURN

END 
SUBROUT INE FITH (IFIELD,LRISE, LFALL)

COMMON/CALC/H(2008), XI $(20), Y 1(3)$, W 120$)$

COMMON/FIELD/DUM(4), H2O(20)

DCUBLE PRECISICN $Q(6)$

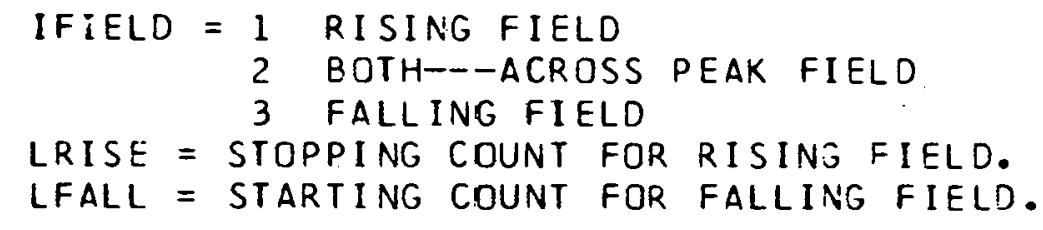

2 IFIELD $=3$ NUMB $=20 C 8$

3 DO $4 K=1,3$ $w(K)=1$.

$5 \times 1(1)=42$. 
$W(1)=1$

DO $6 \quad I=2,20$

$X 1(I)=X 1(I-1)+100$.

$6 W(I)=1$.

IF (I2.GT. 5$)$ GO TO 7

IFIELD $=3$

CO $106 \mathrm{~J}=1$, NUMB

$106 H(J)=1.0$

GO TO 11

7 CALL OPLSPA $15, \mathrm{I} 2, \mathrm{X} 1, \mathrm{H} 20, \mathrm{~W}, \mathrm{Q}, 0.01$ $N 1=1$

$N 2=N U M B$

$80010 \quad I=N 1, N 2$

$X=F L O A T(I)$

$K=2$

$H(I)=Q(1)$

$9 H(I)=H(I)+Q(K) \neq X$

IF $(K . G E .6)$ GO TO 10

$K=K+1$

$X=X * F L G \Delta T(I)$

GO TO 9

10 CONT INUE

IF (NI.NE. I) GO TO 14

IF (IFIELD.NE. 2) GO TO 14

11 I $3=20-I 2$

IF $(13 . G E .5)$ GC TO 12

I $F I E L D=1$

$N 3=N U M B+1$

DO $111 \mathrm{~J}=\mathrm{N} 3,2008$

$111+(J)=1.0$

GO TO 14

$120013 \quad I=1,13$

$L=I+I 2$

$H 20 .(I)=H 20(L)$

$13 \times 1(I)=X 1(L)$

CALL DPLSPA $(5, I 3, X 1, H 20, W, Q, 0.0)$

$N 1=N U M B+1$ 
$N 2=2008$

GO TO 8

14 IF (IFIELD .GT. 2) GO TO 17

$I=N U M B-100$

15 II $I=I+1$

IF (HII) . GE. HIIII) GO TO 16

$I=I I$

IF $(I-N U M B) \quad 15,16,16$

16 LRISE =I

IF IIFIELD .LT. 2 GO TO 20

$17 \mathrm{I}=\mathrm{N} 1+100$

18 II $I=I-1$

IF (H(II) L LE. H(I)) GO TO 19

$I=I I$

IF $(I-N 1) \quad 19,19,18$

19 LFALL $=I$

20 RETURN

ENCI 
SUBROUT INE RANGE (IFIELD, MFICT, MFFCT, NFICT, NFFCT, LORISE, LRISE,

1LFALL, - OFALL, OMEGAI, OMEGA2, DT, RECYC)

COMMON/ AUTOF/FF(20), FL (20), NSTART (20), NS TOP(20)

COMMON/CALC/HI $(2008)$

$F F(I)=$ INITIAL FREQ. ARRAY. FLII) = FINAL FREQ. ARRAY.

NSTARTII), NSTOP(I) ARE STARTING AND STOPPING COUNTS FOR I TH FREQ. RANGE. RECYC IS SET $=0.0$ IF NO ERROR, = 1.0 IF ERROR OCCURS.

IFIELD $=1$ RISING FIELD, = 2 BOTH, = 3 FALLING FIELD.

MFICT,MFFCT ARE STARTING $\&$ STOPPING VALUES FOR SUBSCRIPT I FOR RISING FIELD.

NFICT,NFFET ARE STARTING \& STOPPING VALUES FOR SUBSCRIPT I FOR FALLING FIELD. LORISE AND LRISE ARE COMPUTED START AND STOP COUNTS FOR RISING FIELD.

LFALL AND LOFALL ARE COMPUTED START AND STOP COUNTS FOR FALLING FIELO.

CMEGAl ANO OMEGA2 ARE LOW AND HIGH CUTOFF FREQUENCIES OF AMPLIFIER .

DT IS THE TIME INTERVAL.

IF (IFIELD . GT . 2) GO TO 10

$I=M F I C T$

$1 J=L O R I S E$

$2 O M E G=A 3 S(F F(I) *(H I(J+1)-H I(J)) / D T)$

IF (OMEG .LE. OMEGAL) GO TO 3

$J=j+5$

IF (J-LRISE) $\quad 2,19,19$

3 NSTART $(I)=J$

$I=I+1$

IF (I.GT. MFFCT) GO TO 4

IF $(F F(I)-F F(I-1)) 1,2,2$

$4 I=M F F C T$

$5 J=L R I S E$

6 CMEG $=A B S(F L(I) *(H I(J)-H I(J-1)) / D T)$

IF (OMEG . GE. CMEGAI) GO TO 7

$J=J-5$

IF $(J-L O R I S E) \quad 19,19,6$

7 NSTOP (I ) $=J$

$I=1-1$

IF (I :LT. MFICT) GO TO 10

IF $(F L(I)-F L(I+I)) \quad 6,6,5$ 
10 IF (IFEELD .LT.2) GO TO 18 $I=N F F C T$

$11 \mathrm{~J}=\mathrm{LFALL}$

$12 C M E G=A B S(F L(I) *(H I(J+1)-H I(J)) / O T)$ IF (CMEG . GE. CMEGAI) GO TO. 13 $J=J+5$

IF (J-LOFALL) $12,19,19$

13 NSTART:I $=$ J

$I=I-1$

IF (I .LT. NFICT) GO TO 14

IF $(F L I I)-F L(I+1)) \quad 12,12,11$

$14 \mathrm{I}=\mathrm{NF} I \mathrm{CT}$

$15 \mathrm{~J}=$ LOFALL

$16 O M E G=A B S(F F(I) *(H I(J)-H I(J-1)) / D T)$ IF (OMEG . LE. CMEGA2) GO TO 17 $J=J-2$

IF (J-LFALL) $19,19,16$

17 NSTOP(I) $=\mathrm{J}$ $I=I+I$

IF (I . GT. NFFCT) GO TO 18

IF $(F F(I)-F F(I-1)) \quad 15,16,16$

18 RECYC $=0.0$ GO TO 20

19 RECYC $=1.0$

20 RETURN

ENO 
SUBROUT INE GENY (MM, ID, DRH, NPTS, RES, MF, ML, CZERO, DHI, ARES)

DOUBLE PR.ECISICN HH, DQH,A

COMMON/ AUTOF/FF( 20$)$, FL $(20)$, NSTART $(20)$, NSTDP (20)

CCMMCN/CALC/HI (2008), Y.1(2008)

COMMCN/GIANT/Y(1)

LMIN $=N S T A R T$ (I D)

LMAX $=$ NSTOP (ID)

$D H I=H I(L M I N)-H I(L M A X)$

$C Z E R G=1.0$

IF (RES .EQ. 0.0$)$ GO TO 1

$\triangle R E S=R E S$

$C F I R S T=A B S(F F(I D) * D H I)$

CRES $=1: / R E S$

C ADDED ZEROES NEEDED TO INCREASE RESOLUTION

$M M M=M M *$ (CFIRST/CRES)

IF (MMM . GT. 6384) GO TO 61

$M M M=6384$

ARES $=$ FLOAT $(M M M) /(F L O A T(M M) * C F I R S T)$

$61 D Q H=D H 1 /(M M M-1)$

IF (DQH) $62,681,63$

$c$

FALLING FIELD CASE

$62 \mathrm{MF}=1$

$M L=M M-M M M$

$L F=M L+1$

$L L=M M$

GO TO 64

$c$

RISING FIELD CASE.

$63 L F=1$

$L L=M M M$

$M F=L L+1$

$M L=M M$

C FILL PART DF Y ARRAY WITH ZEROES

$64 D C 65$ I $=$ MF, ML

$65 Y(I)=0.0$

CZERO = FLOAT $(M M M) / F L O A T(M M)$

GE TO 2 
$1 D Q H=D H I /(M M-1)$

$L F=1$

$L L=M M$

2 IF (DQH) $682,681,683$

681 WRITE $(3,100)$

100 FERMAT 1 (' DQH $=0$ ')

GO TO 1014

$682 D Q H=-D Q H$

$A S I N=-1$.

GO TO 1002

683 ASIN $=+1$

$1002 \mathrm{I}=\mathrm{LMIN}$

$1003 \mathrm{~K}=\mathrm{LF}$

$1004 \mathrm{HH}=\mathrm{HI}(1)$

$1005 A=H I(I)$

$34 \mathrm{G}=\mathrm{HH}-\mathrm{A}$

$Y(K)=Y I(I)+(Y 1(I+I)-Y I(I)) G /(H I(I+I)-H I(I))$

$1006 K=K+1$

1007 IF $(K-L L) \quad 35,35,50$

35 IF (ASIN) $39,39,36$

$36 \mathrm{HH}=\mathrm{HH}-\mathrm{OQH}$

$1008 \mathrm{Gl}=\mathrm{HH}$

1009 IF $(G I-H I(I+1)) 40,34,34$

$39 \mathrm{HH}=\mathrm{HH}+\mathrm{DQH}$

$1010 \mathrm{Gl}=\mathrm{HH}$

1011 IF $(G I-H I(I+1)) 34,34,40$

$40 \quad I=I+1$

$1012 A=H I(I)$

1013 IF $(1-L$ MAX $) 41,50,50$

41 IF IHI (I+I)-HI (I)) $34,40,34$

50 NPTS $=K-1$

1014 DRH=DQH

69 RETURN

END 
SUBROUTINE OUTPUT (ID,DRH, NPTS, NGRAPH, ARES, ME, ML, CZERO, DHI, ALIM, INTOUT, NSER I

CCMMON/AUTOF/FF(20), FL(20)

COMMON/CALC/Z(300), UU (300), Z1(150), UUI (150),ZIR(150)

CCMMONIEIANT/Y (1)

COMMON/LABS/GLAB I (5), GLAB2 (5), XLAB 1 (5), YLABI(5), DDATE (7)

NZIMAX $=150$

$F A C T O R=A B S(C Z E R O / D H I)$

KFIRST $=F F($ I O) / FACTOR

$K L A S T==L(I D) / F A C T O P$.

WRITE $(3,508)$ KFIRST,KLAST

IF (KFIRST.GE. KLAST) GO TO 461

WRITE $(3,3333)$ DRH, NPTS,FACTOR

3333

FORMAT $1 . \quad D R H=1, E 16.7,1$

IF (CZERO .EQ. 1.0) GO TO 10

RRES $=A R E S * 100$.

$M F T=M L-N F+1$

WRITE $(3,110) \quad M F T, M F, M L, R R E S$

110 FORMAT ('0', I 13,' ZEROES WERE ADDED IN Y-ARRAY BETWEEN', I6,' AND',

116,1 TO OBTAIN RESOLUTION OF',F6.3,' PER CENT.''

10 SCAN $=299$

510 IF (KLAST-KFIRST.GT. SCAN) GO TO 200

KSTART $=$ KFIRST

$K S T O P=K L A S T$

502 NFIRST $=(K S T A R T * 2)+1$

$N L A S T=(K S T O P * 2)+1$

$K F I R S T=K F I R S T+S C A M-10$

$J=0$

DO $500 \quad K=N F I R S T, N L A S T, 2$

$\mathrm{J}=\mathrm{J}+1$

$Z(J)=(Y(K)) * 2+(Y(K+1)) * * 2$

$W=(K-1) / 2$

500 UU $(J)=F A C T O R * W$

CUUL $=$ UU $(1) / 1 . E 06$

CUUJ $=U U(\mathrm{~J}) / 1 . E 06$

WRITE $(3,601)$ CUU1 


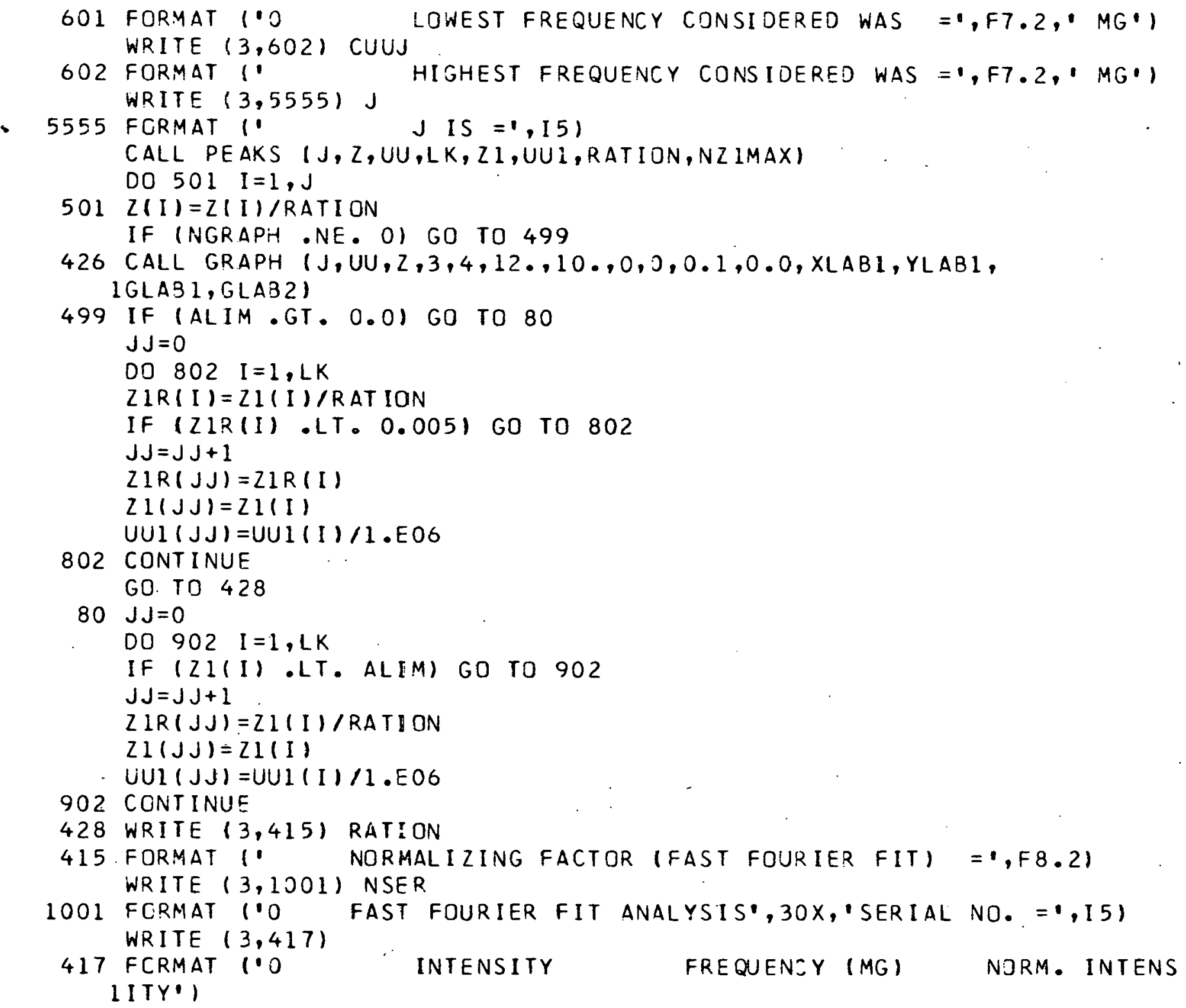


WRITE $(3,419)$

419 FORMAT 1 ' ')

IF IJJ.EQ. OI GO TO 1070

820 DO $420 \quad I=1, J J$

420 WRITE $(3,1006), 21(I), U U 1(I), Z 1 R(I)$

1006 FORMAT (3F18.3)

IF (NTOUT .EQ. O) GO TO 1070

WRITE (8) NSER, GLA91, GLAB2, DDATE, CUU1, CUUJ, JJ

WRITE (8) $((Z 1(I), I=1, J J),(U \cup I(K), K=1, J J))$

NSER $=$ NSER+1

1070 WRITE $(3,416)$

416 FORMAT $(1001)$

IF (KFIRST - KLAST) $510,461,461$

$200 K S T A R T=K F I R S T$

KSTOP $=K$ START+ SCAN

GO TO 502

461 RETURN

END 


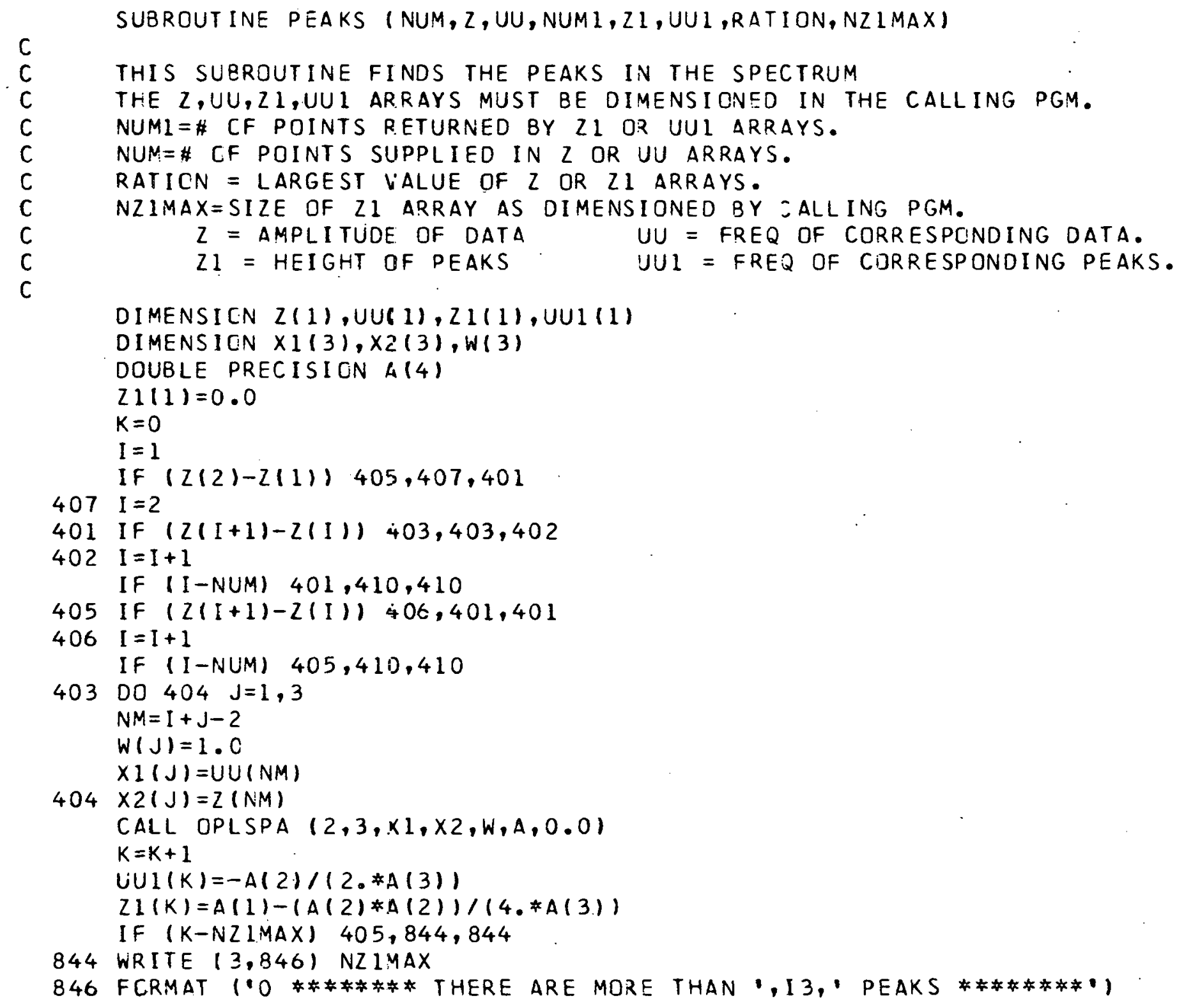




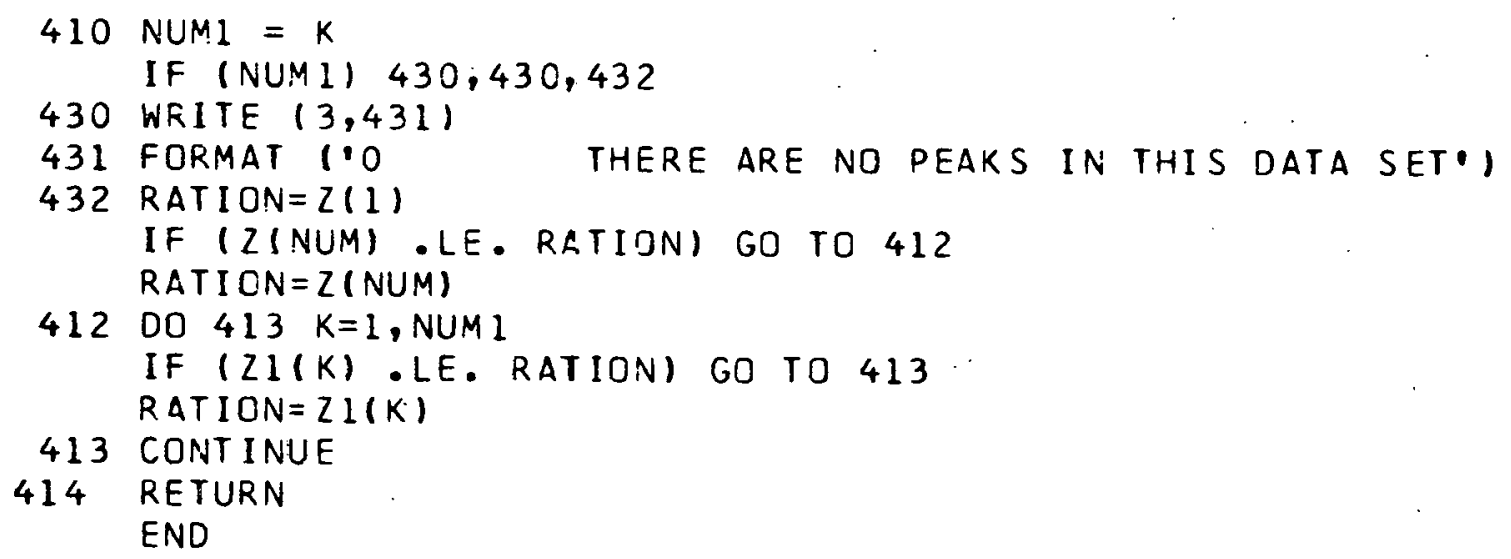




\section{COMMENTS ON LIBRARY PROGRAMS CALLED:}

\section{DATE}

DIMENSICN $\times(7)$

CALL DATE $(X)$

RESULTS IN CURRENT DATE AND TIME IN $7 A 4$ FORMAT.

STARTM \& STOPTM

$A=0.0$

CALL STARTM $(A, B)$

* * otHER pROgRAM STATEMENTS

CALL STCPTM $(A, B)$

RESULTS IN A = REAL TIME ELAPSED BETWEEN CALLS TO STARTM \& STOPTM. RESUITS IN $B=$ CPU TIME ELAPSED BETWEEN CALLS TO STARTM E STOPTM.

\section{OPL SPA}

OPLSPA IS A ROUTINE TO LEAST SQUARES FIT A POLYNOMIAL OF DESIRED DEGREE TO GIVEN DATA.

DOUBLE PRECISION G (NDEG+1)

CALL OPLSPA INDEG, NPTS $, X, Y, W, Q, 0.01$

NDEG = DEGREE OF DESIRED POLYNOMIAL = MAX. VALUE OF 10.

NPTS = NUMBER OF POINTS.

$X=F I R S T$ ELEMENT OF TABLE OF INDEPENDENT VARIABLES.

$Y=F I R S T$ ELEMENT OF TABLE OF DEPENDENT VARIABLES.

$W=$ FIRST ELEMENT OF TABLE OF WEIGHTS ASSOCI ATED WITH THE CORRESPCNDING DATA POINT X(I),Y(I). IF DATA IS UNWEIGHTED, SET $W(I)=1.0$ FOR $I=1$ TO NPTS.

$Q=$ FIRST ELEMENT OF TABLE OF COEFFICIENTS RETURNED BY OPLSPA. TABLE ARRANGED TO CORRESPOND TO ASCENDING POWERS OF

THE INOEPENDENT VARIABLE. 